\title{
Indium-Copper Mediated Barbier-Grignard-Type Alkylation Reaction of Imines in Aqueous Media
}

\author{
Zhi-Liang Shen and Teck-Peng Loh* \\ Division of Chemistry and Biological Chemistry, School of Physical and Mathematical Sciences, \\ Nanyang Technological University, Singapore 639798
}

Supporting Information

$\underline{\text { Table of contents }}$

General methods...............................................................S2

Experimental procedure.........................................................S3

Spectroscopic data of products.....................................................S4

${ }^{1} \mathrm{H}$ and ${ }^{13} \mathrm{C}$ NMR Spectra of Products............................................S16 


\section{$\underline{\text { General methods }}$}

All aldehydes were purified before using. All commercially available amines (non-chiral amine) and alkyl iodides were used directly without purification. Commercially available chiral amines (hydrochloride form) were washed with saturated aqueous sodium carbonate solution before using.

Following commercial grade solvents and reagents were also used without further purification: indium (powder, -100 mesh, 99.99\%, Aldrich chemicals), copper (I) iodide (98\%, Alfa Aesar chemical), indium trichloride (strem chemical).

Deionised water was used in all reactions.

The stirrer was "Mr 3001K" type, purchased from Heidolph. It was stirred at the largest speed of $1250 \mathrm{rpm}$. The stirring bar was egg-shaped in $1.6 \mathrm{~cm}$ x $0.6 \mathrm{~cm}$ (L x Diam.).

Analytical thin layer chromatography (TLC) was performed using Merck 60 F254 precoated silica gel plate $(0.2 \mathrm{~mm}$ thickness). Subsequent to elution, plates were visualized using UV radiation $(254 \mathrm{~nm})$ on Spectroline Model ENF-24061/F $254 \mathrm{~nm}$. Further visualization was possible by staining with acidic solution of ceric molybdate or ethanol solution of ninhydride.

Flash chromatography was performed using Merck silica gel 60 with freshly distilled solvents. Columns were typically packed as slurry and equilibrated with the appropriate solvent system prior to use.

Infrared spectra were recorded on a Bio-Rad FTS 165 FTIR spectrometer. The oil samples were examined under neat conditions.

High Resolution Mass (HRMS) spectra were obtained using Finnigan MAT95XP GC/HRMS (Thermo Electron Corporation).

Proton nuclear magnetic resonance spectra $\left({ }^{1} \mathrm{H}\right.$ NMR) were recorded on a Bruker Avance DPX 300 and Bruker AMX 400 spectrophotometer ( $\mathrm{CDCl}_{3}$ as solvent). Chemical shifts for ${ }^{1} \mathrm{H}$ NMR spectra are reported as $\delta$ in units of parts per million (ppm) downfield from $\mathrm{SiMe}_{4}(\delta 0.0)$ and relative to the signal of chloroform- $d(\delta 7.2600$, singlet). Multiplicities were given as: s (singlet); $\mathrm{d}$ (doublet); $\mathrm{t}$ (triplet); q (quartet); or m (multiplets). The number of protons (n) for a given resonance is indicated by $\mathrm{nH}$. Coupling constants are reported as a $J$ value in $\mathrm{Hz}$. Carbon nuclear magnetic resonance spectra $\left({ }^{13} \mathrm{C} \mathrm{NMR}\right)$ are reported as $\delta$ in units of parts per million (ppm) downfield from $\mathrm{SiMe}_{4}(\delta 0.0)$ and relative to the signal of chloroform- $d$ ( $\delta 77.03$, triplet). The proportion of diastereomers was determined from the integration of ${ }^{1} \mathrm{H} N M R$ and ${ }^{13} \mathrm{C}$ NMR spectra. 


\section{Experimental procedure}

For the alkylation reaction of imines which derived from aromatic amine and aromatic aldehyde: To a $10 \mathrm{~mL}$ round-bottomed flask was added water $(10 \mathrm{~mL})$, aldehyde $(0.5 \mathrm{mmol})$, amine $(0.6 \mathrm{mmol})$, and $\mathrm{InCl}_{3}(0.05 \mathrm{mmol})$, stirred for 10 minutes at room temperature, then indium ( $3 \mathrm{mmol}$ ), copper iodide ( $2 \mathrm{mmol}$ ), and alkyl iodide $(2.5 \mathrm{mmol})$ were added to the reaction system sequentially, then it was stirred vigorously at room temperature for 1 day. After reaction, it was extracted using diethyl ether (20 $\mathrm{mL}$ x 3), washed with brine, dried over anhydrous sodium sulfate, filtered and evaporated solvent under vacuo to give the residue. It was subjected to silica gel column chromatography using ethyl acetate and hexane as eluent to afford the desired product.

For the alkylation reaction of imines which derived from aliphatic amine (including benzyl amine and chiral amines): To a $10 \mathrm{~mL}$ round-bottomed flask was added $\mathrm{MeOH}(5 \mathrm{~mL}), \mathrm{H}_{2} \mathrm{O}(5$ $\mathrm{mL})$, aldehyde $(0.5 \mathrm{mmol})$, amine $(1.0 \mathrm{mmol})$, and $\mathrm{InCl}_{3}(0.05 \mathrm{mmol}$ for benzyl amine, $0.5 \mathrm{mmol}$ for chiral amines), stirred for 4 hrs at room temperature. Then indium ( $3 \mathrm{mmol}$ ), copper iodide (2 mmol), and alkyl iodide ( $2.5 \mathrm{mmol})$ were added to the reaction system sequentially, and stirred vigorously at room temperature for 1 day. After reaction, it was extracted using diethyl ether (20 $\mathrm{mL} \times 3$ ), washed with brine, dried over anhydrous sodium sulfate, filtered and evaporated solvent under vacuo to give the residue. It was subjected to silica gel column chromatography using ethyl acetate and hexane (with $1 \% \mathrm{v} / \mathrm{v}$ triethyl amine) as eluent to afford the desired product. 


\section{Spectroscopic data of products}

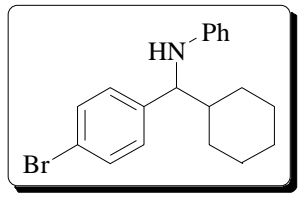

$N$-Phenyl-[1-(p-bromophenyl)-1-cyclohexyl-methyl]amine (Table 2, entry 1): Light yellow oil; Yield: 84\%; $\mathrm{R}_{f}=0.61$ (Ethyl acetate/Hexane 1:8); FTIR (NaCl, neat): $v 3422(\mathrm{NH}) \mathrm{cm}^{-1} ;{ }^{1} \mathrm{H}$ NMR (300 MHz, CDCl $): \delta 0.83-1.26(\mathrm{~m}, 6 \mathrm{H}), 1.49-1.85$ (m, 5H), 4.05 (d, $J=6.03 \mathrm{~Hz}, 1 \mathrm{H}), 4.09$ (s, br, 1H), 6.44 (d, $J=7.80 \mathrm{~Hz}, 2 \mathrm{H}), 6.60$ (t, $J=7.32 \mathrm{~Hz}, 1 \mathrm{H}), 7.02-7.07(\mathrm{~m}, 2 \mathrm{H}), 7.13-7.17(\mathrm{~m}$, 2H), 7.37-7.40 (m, 2H) ppm; ${ }^{13} \mathrm{C}$ NMR (100 MHz, $\mathrm{CDCl}_{3}$ ): $\delta 147.4$ (C), 141.7 (C), 131.3 (CHx2), 129.1 (CHx2), 120.4 (C), 128.9 (CHx2), 117.2 (CH), 113.1 (CHx2), $62.9(\mathrm{CH}), 44.7(\mathrm{CH}), 30.1$ $\left(\mathrm{CH}_{2}\right), 29.3\left(\mathrm{CH}_{2}\right), 26.3\left(\mathrm{CH}_{2}\right), 26.3\left(\mathrm{CH}_{2}\right), 26.3\left(\mathrm{CH}_{2}\right)$ ppm; HRMS (EI, m/z): Calcd. for $\mathrm{C}_{19} \mathrm{H}_{22} \mathrm{NBr}$ : 343.0936, found [M-H] $]^{+}: 342.0828$.

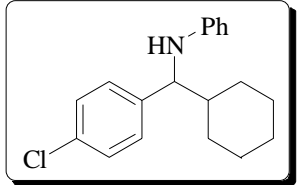

$\boldsymbol{N}$-Phenyl-[1-(p-chlorophenyl)-1-cyclohexyl-methyl]amine (Table 2, entry 2): Light yellow oil; Yield: $65 \%$; $\mathrm{R}_{f}=0.59$ (Ethyl acetate/Hexane 1:8); FTIR (NaCl, neat): $v 3426(\mathrm{NH}) \mathrm{cm}^{-1} ;{ }^{1} \mathrm{H}$ NMR (300 MHz, $\left.\mathrm{CDCl}_{3}\right): \delta$ 0.95-1.29 (m, 5H), 1.42-1.85 (m, 6H), 4.07 (s, 1H), 4.09 (s, 1H), 6.43-6.46 (m, 2H), 6.58-6.63 (m, 1H), 7.02-7.08 (m, 2H), 7.19-7.26 (m, 4H) ppm; ${ }^{13} \mathrm{C}$ NMR (75.4 MHz, $\mathrm{CDCl}_{3}$ ): $\delta 147.4$ (C), 141.2 (C), 132.3 (C), 129.1 (CHx2), 128.6 (CHx2), 128.4 (CHx2), 117.2 (CH), 113.2 (CHx2), $62.8(\mathrm{CH}), 44.8(\mathrm{CH}), 30.1\left(\mathrm{CH}_{2}\right), 29.4\left(\mathrm{CH}_{2}\right), 26.3\left(\mathrm{CH}_{2}\right), 26.3\left(\mathrm{CH}_{2}\right)$, $26.3\left(\mathrm{CH}_{2}\right)$ ppm; HRMS (EI, m/z): Calcd. for $\mathrm{C}_{19} \mathrm{H}_{22} \mathrm{ClN}$ : 299.1441, found [M-H] $]^{+}: 298.1346$.

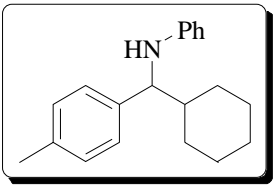

$N$-Phenyl-[1-(p-methylphenyl)-1-cyclohexyl-methyl]amine (Table 2, entry 3): Light yellow oil; Yield: 99\%; $\mathrm{R}_{f}=0.63$ (Ethyl acetate/Hexane 1:8); FTIR (NaCl, neat): $v 3424(\mathrm{NH}) \mathrm{cm}^{-1} ;{ }^{1} \mathrm{H}$ NMR (400 MHz, CDCl $): \delta 0.84-0.90(\mathrm{~m}, 1 \mathrm{H}), 0.95-1.26$ (m, 5H), 1.50-1.74 (m, 4H), 1.85-1.88 (m, 1H), 2.27 (s, 3H), 4.06 (d, $J=6.20 \mathrm{~Hz}, 1 \mathrm{H}), 4.09$ (s, br, 1H), 6.47 (d, $J=7.89 \mathrm{~Hz}, 2 \mathrm{H}), 6.57$ (t, $J=7.20 \mathrm{~Hz}, 1 \mathrm{H}), 7.01-7.15$ (m, 6H) ppm; ${ }^{13} \mathrm{C}$ NMR (100 MHz, $\left.\mathrm{CDCl}_{3}\right): \delta 147.8$ (C), 139.5 (C), 136.1 (C), 129.0 (CHx2), 128.8 (CHx2), 127.1 (CHx2), $116.8(\mathrm{CH}), 113.1$ (CHx2), $63.0(\mathrm{CH})$, $44.9(\mathrm{CH}), 30.2\left(\mathrm{CH}_{2}\right), 29.5\left(\mathrm{CH}_{2}\right), 26.4\left(\mathrm{CH}_{2}\right), 26.4\left(\mathrm{CH}_{2}\right), 26.3\left(\mathrm{CH}_{2}\right), 21.0\left(\mathrm{CH}_{3}\right)$ ppm; HRMS (EI, m/z): Calcd. for $\mathrm{C}_{20} \mathrm{H}_{25} \mathrm{~N}$ : 279.1987, found [M-H] ${ }^{+}$: 278.1897. 


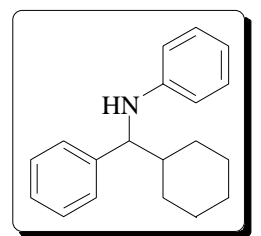

$N$-Phenyl-(1-phenyl-1-cyclohexyl-methyl)amine (Table 2, entry 4): Light yellow oil; Yield: 97\%; $\mathrm{R}_{f}=0.57$ (Ethyl acetate/Hexane 1:8); FTIR (NaCl, neat): $v 3426(\mathrm{NH}) \mathrm{cm}^{-1} ;{ }^{1} \mathrm{H}$ NMR (400 $\left.\mathrm{MHz}, \mathrm{CDCl}_{3}\right): \delta 1.00-1.26$ (m, 5H), 1.47-1.54 (m, 1H), 1.60-1.76 (m, 4H), 1.86-1.89 (m, 1H), 4.10 (d, $J=6.32 \mathrm{~Hz}, 1 \mathrm{H}), 4.12(\mathrm{~s}, \mathrm{br}, 1 \mathrm{H}), 6.48(\mathrm{~d}, J=7.72 \mathrm{~Hz}, 2 \mathrm{H}), 6.59(\mathrm{t}, J=7.44 \mathrm{~Hz}, 1 \mathrm{H})$, 7.02-7.06 (m, 2H), 7.18-7.21 (m, 1H), 7.27-7.28 (m, 4H) ppm; ${ }^{13} \mathrm{C}$ NMR (75.4 MHz, $\left.\mathrm{CDCl}_{3}\right): \delta$ 147.8 (C), 142.7 (C), 129.0 (CHx2), 128.2 (CHx2), 127.2 (CHx2), 126.7 (CH), 116.9 (CH), 113.1 (CHx2), $63.4(\mathrm{CH}), 44.9(\mathrm{CH}), 30.2\left(\mathrm{CH}_{2}\right), 29.4\left(\mathrm{CH}_{2}\right), 26.4\left(\mathrm{CH}_{2}\right), 16.4\left(\mathrm{CH}_{2}\right), 26.3\left(\mathrm{CH}_{2}\right) \mathrm{ppm}$; HRMS (EI, m/z): Calcd. for $\mathrm{C}_{19} \mathrm{H}_{23} \mathrm{~N}$ : 265.1830, found [M] ${ }^{+}: 265.1810$.

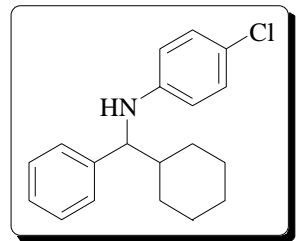

$\mathrm{N}$-(p-Chlorophenyl)-(1-phenyl-1-cyclohexyl-methyl)amine (Table 2, entry 5): Light yellow oil; Yield: 68\%; $\mathrm{R}_{f}=0.62$ (Ethyl acetate/Hexane 1:8); FTIR (NaCl, neat): $v 3428(\mathrm{NH}) \mathrm{cm}^{-1} ;{ }^{1} \mathrm{H}$ NMR (300 MHz, $\mathrm{CDCl}_{3}$ ): $\delta$ 0.93-1.28 (m, 5H), 1.48-1.52 (m, 1H), 1.56-1.75 (m, 4H), 1.83-1.88 (m, 1H), 4.04 (d, $J=6.16 \mathrm{~Hz}, 1 \mathrm{H}$ ), 4.15 (s, br, 1H), 6.39 (d, $J=8.79 \mathrm{~Hz}, 2 \mathrm{H}), 6.97$ (d, $J=8.79 \mathrm{~Hz}$, 2H), 7.16-7.29 (m, 5H) ppm; ${ }^{13} \mathrm{C}$ NMR (75.4 MHz, $\mathrm{CDCl}_{3}$ ): $\delta 146.2$ (C), 142.0 (C), 128.8 (CHx2), 128.2 (CHx2), 127.1 (CHx2), 126.9 (CH), 121.4 (C), 114.2 (CHx2), $63.4(\mathrm{CH}), 44.7$ (CH), 30.1 $\left(\mathrm{CH}_{2}\right), 29.4\left(\mathrm{CH}_{2}\right), 26.3\left(\mathrm{CH}_{2}\right), 26.3\left(\mathrm{CH}_{2}\right), 26.2\left(\mathrm{CH}_{2}\right) \mathrm{ppm}$; HRMS (EI, m/z): [M] ${ }^{+}$, Calcd. for $\mathrm{C}_{19} \mathrm{H}_{22} \mathrm{ClN}$ : 299.1441, found: 299.1432.

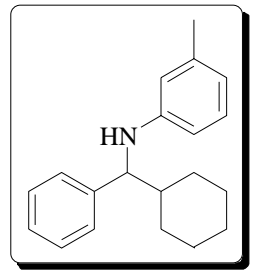

$\mathbf{N}$-(m-Methylphenyl)-(1-phenyl-1-cyclohexyl-methyl)amine (Table 2, entry 6): Light yellow oil; Yield: 86\%; $\mathrm{R}_{f}=0.61$ (Ethyl acetate/Hexane 1:8); FTIR (NaCl, neat): $v 3381(\mathrm{NH}) \mathrm{cm}^{-1} ;{ }^{1} \mathrm{H}$ NMR (300 MHz, $\left.\mathrm{CDCl}_{3}\right): \delta 1.01-1.23(\mathrm{~m}, 5 \mathrm{H}), 1.49-1.53(\mathrm{~m}, 1 \mathrm{H}), 1.62-1.71(\mathrm{~m}, 4 \mathrm{H}), 1.84-1.88$ (m, 1H), 2.17 (s, 3H), 4.08-4.10 (m, 2H), 6.27 (d, $J=7.90 \mathrm{~Hz}, 1 \mathrm{H}), 6.34$ (s, 1H), 6.41 (d, $J=7.30$ $\mathrm{Hz}, 1 \mathrm{H}), 6.92$ (t, $J=7.70 \mathrm{~Hz}, 1 \mathrm{H}), 7.14-7.27(\mathrm{~m}, 5 \mathrm{H}) \mathrm{ppm} ;{ }^{13} \mathrm{C} \mathrm{NMR}\left(75.4 \mathrm{MHz}, \mathrm{CDCl}_{3}\right): \delta 147.8$ (C), 142.8 (C), 138.6 (C), 128.9 (CH), 128.1 (CHx2), 127.1 (CHx2), $126.6(\mathrm{CH}), 117.9(\mathrm{CH})$, $114.0(\mathrm{CH}), 110.1(\mathrm{CH}), 63.3(\mathrm{CH}), 44.9(\mathrm{CH}), 30.2\left(\mathrm{CH}_{2}\right), 29.4\left(\mathrm{CH}_{2}\right), 26.4\left(\mathrm{CH}_{2}\right), 26.3\left(\mathrm{CH}_{2}\right)$, $26.3\left(\mathrm{CH}_{2}\right)$, $21.6\left(\mathrm{CH}_{3}\right)$ ppm; HRMS (EI, m/z): Calcd. for $\mathrm{C}_{20} \mathrm{H}_{25} \mathrm{~N}$ : 279.1987, found $[\mathrm{M}-\mathrm{H}]^{+}$: 278.1901. 


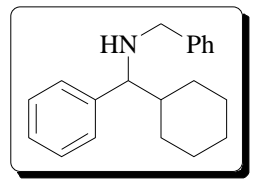

$N$-Benzyl-(1-phenyl-1-cyclohexyl-methyl)amine (Table 2, entry 7): Light yellow oil; Yield: 77\%; $\mathrm{R}_{f}=0.41$ (Ethyl acetate/Hexane 1:8); FTIR ( $\mathrm{NaCl}$, neat): $v 3431(\mathrm{NH}) \mathrm{cm}^{-1} ;{ }^{1} \mathrm{H}$ NMR (300 MHz, $\left.\mathrm{CDCl}_{3}\right): \delta 0.80-1.26(\mathrm{~m}, 5 \mathrm{H}), 1.37-1.41(\mathrm{~m}, 1 \mathrm{H}), 1.48-1.73(\mathrm{~m}, 5 \mathrm{H}), 1.94-1.98(\mathrm{~m}, 1 \mathrm{H}), 3.35$ (d, $J=7.18 \mathrm{~Hz}, 1 \mathrm{H}), 3.43$ (d, $J=13.24 \mathrm{~Hz}, 1 \mathrm{H}), 3.62(\mathrm{~d}, J=13.25 \mathrm{~Hz}, 1 \mathrm{H}), 7.18-7.34(\mathrm{~m}, 10 \mathrm{H})$ ppm; ${ }^{13} \mathrm{C}$ NMR (75.4 MHz, $\mathrm{CDCl}_{3}$ ): $\delta 143.0$ (C), 140.9 (C), 128.2 (CHx2), 128.1 (CHx4), 128.0 (CHx2), 126.7 (CHx2), $68.0(\mathrm{CH}), 51.6\left(\mathrm{CH}_{2}\right), 44.3(\mathrm{CH}), 30.2\left(\mathrm{CH}_{2}\right), 29.9\left(\mathrm{CH}_{2}\right), 26.5\left(\mathrm{CH}_{2}\right)$, $26.3\left(\mathrm{CH}_{2}\right), 26.3\left(\mathrm{CH}_{2}\right)$ ppm; HRMS (EI, m/z): Calcd. for $\mathrm{C}_{20} \mathrm{H}_{25} \mathrm{~N}$ : 279.1987, found $[\mathrm{M}-\mathrm{H}]^{+}$: 278.1903.

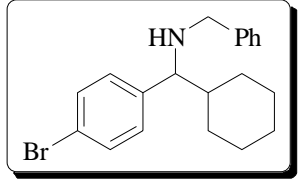

$N$-Benzyl-[1-(p-bromophenyl)-1-cyclohexyl-methyl]amine (Table 2, entry 8): Light yellow oil; Yield: 70\%; $\mathrm{R}_{f}=0.47$ (Ethyl acetate/Hexane 1:8); FTIR (NaCl, neat): $v 3350(\mathrm{NH}) \mathrm{cm}^{-1} ;{ }^{1} \mathrm{H}$ NMR (300 MHz, CDCl $): \delta 0.78-1.20$ (m, 5H), 1.37-1.73 (m, 6H), 1.88-1.92 (m, 1H), 3.33 (d, $J=$ $6.88 \mathrm{~Hz}, 1 \mathrm{H}$ ), 3.41 (d, $J=13.27 \mathrm{~Hz}, 1 \mathrm{H}$ ), 3.60 (d, $J=13.23 \mathrm{~Hz}, 1 \mathrm{H}), 7.15$ (d, $J=8.24 \mathrm{~Hz}, 2 \mathrm{H}$ ), 7.22-7.31 (m, 5H), 7.44 (d, $J=8.28 \mathrm{~Hz}, 2 \mathrm{H}) \mathrm{ppm} ;{ }^{13} \mathrm{C}$ NMR (75.4 MHz, $\mathrm{CDCl}_{3}$ ): $\delta 142.1(\mathrm{C})$, 140.7 (C), 131.1 (CHx2), 129.9 (CHx2), 128.3 (CHx2), 128.1 (CHx2), 126.8 (CH), 120.4 (C), $67.4(\mathrm{CH}), 51.6\left(\mathrm{CH}_{2}\right), 44.3(\mathrm{CH}), 30.1\left(\mathrm{CH}_{2}\right), 29.7\left(\mathrm{CH}_{2}\right), 26.5\left(\mathrm{CH}_{2}\right), 26.3\left(\mathrm{CH}_{2}\right), 26.2\left(\mathrm{CH}_{2}\right)$ ppm; HRMS (EI, m/z): Calcd. for $\mathrm{C}_{20} \mathrm{H}_{24} \mathrm{BrN}$ : 357.1092, found [M-H] ${ }^{+}: 356.1000$.

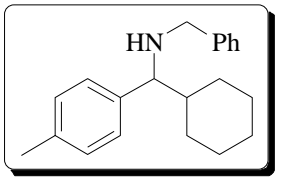

$N$-Benzyl-[1-(p-methylphenyl)-1-cyclohexyl-methyl]amine (Table 2, entry 9): Light yellow oil; Yield: 81\%; $\mathrm{R}_{f}=0.37$ (Ethyl acetate/Hexane 1:8); FTIR (NaCl, neat): $v 3402(\mathrm{NH}) \mathrm{cm}^{-1} ;{ }^{1} \mathrm{H}$ NMR (300 MHz, $\left.\mathrm{CDCl}_{3}\right): \delta 0.75-1.26(\mathrm{~m}, 5 \mathrm{H}), 1.38-1.72(\mathrm{~m}, 6 \mathrm{H}), 1.94-1.98(\mathrm{~m}, 1 \mathrm{H}), 2.34(\mathrm{~s}, 3 \mathrm{H})$, 3.32 (d, $J=7.12 \mathrm{~Hz}, 1 \mathrm{H}), 3.43$ (d, $J=13.23 \mathrm{~Hz}, 1 \mathrm{H}), 3.62$ (d, $J=13.24 \mathrm{~Hz}, 1 \mathrm{H}), 7.10-7.31$ (m, 9H) ppm; ${ }^{13} \mathrm{C}$ NMR (75.4 MHz, $\mathrm{CDCl}_{3}$ ): $\delta 141.0$ (C), 139.9 (C), 136.1 (C), 128.7 (CHx2), 128.2 (CHx2), 128.1 (CHx2), $128.0(\mathrm{CHx} 2), 126.7(\mathrm{CH}), 67.7(\mathrm{CH}), 51.6\left(\mathrm{CH}_{2}\right), 44.3(\mathrm{CH}), 30.3\left(\mathrm{CH}_{2}\right)$, $29.9\left(\mathrm{CH}_{2}\right), 26.6\left(\mathrm{CH}_{2}\right), 26.4\left(\mathrm{CH}_{2}\right), 26.3\left(\mathrm{CH}_{2}\right), 21.1\left(\mathrm{CH}_{3}\right)$ ppm; HRMS (EI, m/z): Calcd. for $\mathrm{C}_{21} \mathrm{H}_{27} \mathrm{~N}: 293.2143$, found [M-H] $]^{+}: 292.2065$.

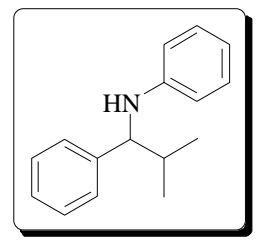


$N$-Phenyl-(2-methyl-1-phenyl-propyl)amine (Table 2, entry 10): Light yellow oil; Yield: 88\%; $\mathrm{R}_{f}=0.63$ (Ethyl acetate/Hexane 1:8); FTIR ( $\mathrm{NaCl}$, neat): $v 3426(\mathrm{NH}) \mathrm{cm}^{-1} ;{ }^{1} \mathrm{H}$ NMR $(400 \mathrm{MHz}$, $\left.\mathrm{CDCl}_{3}\right): \delta 0.91$ (d, $\left.J=6.79 \mathrm{~Hz}, 3 \mathrm{H}\right), 0.97(\mathrm{~d}, J=6.77 \mathrm{~Hz}, 3 \mathrm{H}), 1.98-2.06(\mathrm{~m}, 1 \mathrm{H}), 4.10(\mathrm{~s}, 1 \mathrm{H})$, 4,12 (s, 1H), 6.49 (d, $J=7.88 \mathrm{~Hz}, 2 \mathrm{H}), 6.60$ (t, $J=7.26 \mathrm{~Hz}, 1 \mathrm{H}), 7.03-7.07$ (m, 2H), 7.17-7.22 (m, 1H), 7.26-7.29 (m, 4H) ppm; ${ }^{13} \mathrm{C}$ NMR (100 MHz, $\left.\mathrm{CDCl}_{3}\right): \delta 147.7$ (C), 142.6 (C), 129.0 (CHx2), 128.2 (CHx2), 127.2 (CHx2), $126.8(\mathrm{CH}), 117.0(\mathrm{CH}), 113.2$ (CHx2), $63.8(\mathrm{CH}), 34.9(\mathrm{CH}), 19.7$ $\left(\mathrm{CH}_{3}\right)$, $18.6\left(\mathrm{CH}_{3}\right)$ ppm; HRMS (EI, m/z): Calcd. for $\mathrm{C}_{16} \mathrm{H}_{19} \mathrm{~N}$ : 225.1517, found $[\mathrm{M}-\mathrm{H}]^{+}$: 224.1434 .

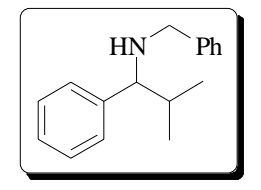

$N$-Benzyl-(2-methyl-1-phenyl-propyl)amine (Table 2, entry 11): Light yellow oil; Yield: 65\%; $\mathrm{R}_{f}=0.30$ (Ethyl acetate/Hexane 1:8); FTIR ( $\mathrm{NaCl}$, neat): $v 3401(\mathrm{NH}) \mathrm{cm}^{-1} ;{ }^{1} \mathrm{H}$ NMR (400 MHz, $\left.\mathrm{CDCl}_{3}\right): \delta 0.74(\mathrm{~d}, J=6.80 \mathrm{~Hz}, 3 \mathrm{H}), 0.97(\mathrm{~d}, J=6.67 \mathrm{~Hz}, 3 \mathrm{H}), 1.81$ (s, br, $\left.1 \mathrm{H}\right), 1.84-1.92(\mathrm{~m}, 1 \mathrm{H})$, 3.34 (d, $J=6.96 \mathrm{~Hz}, 1 \mathrm{H}), 3.46$ (d, $J=13.30 \mathrm{~Hz}, 1 \mathrm{H}$ ), 3.65 (d, $J=13.29 \mathrm{~Hz}, 1 \mathrm{H}), 7.23-7.35$ (m, 10H) ppm; ${ }^{13} \mathrm{C}$ NMR (75.4 MHz, $\mathrm{CDCl}_{3}$ ): $\delta 142.9$ (C), 141.0 (C), 128.3 (CHx2), 128.1 (CHx4), 128.0 (CHx2), $126.8(\mathrm{CH}), 126.8(\mathrm{CH}), 68.7(\mathrm{CH}), 51.7\left(\mathrm{CH}_{2}\right), 34.5(\mathrm{CH}), 19.7\left(\mathrm{CH}_{3}\right), 19.5\left(\mathrm{CH}_{3}\right)$ ppm; HRMS (EI, m/z): Calcd. for $\mathrm{C}_{17} \mathrm{H}_{21} \mathrm{~N}$ : 239.1674, found [M-H] $]^{+}: 238.1589$.

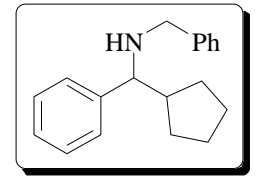

$N$-Benzyl-(1-phenyl-1-cyclopentyl-methyl)amine (Table 2, entry 12): Light yellow oil; Yield: $49 \% ; \mathrm{R}_{f}=0.30$ (Ethyl acetate/Hexane 1:8); FTIR ( $\mathrm{NaCl}$, neat): $v 3418(\mathrm{NH}) \mathrm{cm}^{-1} ;{ }^{1} \mathrm{H}$ NMR (300 MHz, $\left.\mathrm{CDCl}_{3}\right): \delta 1.02-1.10(\mathrm{~m}, 1 \mathrm{H}), 1.23-1.63(\mathrm{~m}, 6 \mathrm{H}), 1.81$ (s, $\left.1 \mathrm{H}\right), 1.90-1.95$ (m, 1H), 2.03-2.11 (m, 1H), 3.32 (d, $J=9.08 \mathrm{~Hz}, 1 \mathrm{H}), 3.43$ (d, $J=13.29 \mathrm{~Hz}, 1 \mathrm{H}), 3.62$ (d, $J=13.29 \mathrm{~Hz}, 1 \mathrm{H}$ ), 7.24-7.32 (m, 10H) ppm; ${ }^{13} \mathrm{C}$ NMR (75.4 MHz, $\mathrm{CDCl}_{3}$ ): $\delta 144.0$ (C), 140.9 (C), 128.3 (CHx2), 128.1 (CHx4), 127.9 (CHx2), $126.8(\mathrm{CH}), 126.7(\mathrm{CH}), 68.1(\mathrm{CH}), 51.5\left(\mathrm{CH}_{2}\right), 47.4(\mathrm{CH}), 30.5$ $\left(\mathrm{CH}_{2}\right), 30.3\left(\mathrm{CH}_{2}\right), 25.3\left(\mathrm{CH}_{2}\right), 25.0\left(\mathrm{CH}_{2}\right)$ ppm; HRMS (EI, m/z): Calcd. for $\mathrm{C}_{19} \mathrm{H}_{23} \mathrm{~N}$ : 265.1830, found $[\mathrm{M}-\mathrm{H}]^{+}: 264.1760$.

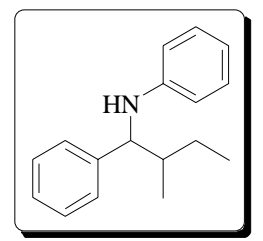

$\mathrm{N}$-Phenyl-(2-methyl-1-phenyl-butyl)amine (Table 2, entry 13): Light yellow oil; Yield: 83\%; $\mathrm{R}_{f}$ $=0.63$ (Ethyl acetate/Hexane 1:8); FTIR ( $\mathrm{NaCl}$, neat): $v 3428(\mathrm{NH}) \mathrm{cm}^{-1} ;{ }^{1} \mathrm{H}$ NMR $(300 \mathrm{MHz}$, $\mathrm{CDCl}_{3}$ ): (major isomer) $\delta$ 0.85-0.94 (m, 6H), 1.10-1.32 (m, 1H), 1.42-1.65 (m, $\left.1 \mathrm{H}\right), 1.76-1.81(\mathrm{~m}$, 1H), 4.07 (s, br, 1H), 4.20 (d, $J=5.82 \mathrm{~Hz}, 1 \mathrm{H}), 6.48$ (d, $J=8.05 \mathrm{~Hz}, 2 \mathrm{H}), 6.57-6.62$ (m, $1 \mathrm{H})$, 7.02-7.08 (m, 2H), 7,17-7.21 (m, 5H) ppm; ${ }^{13} \mathrm{C}$ NMR (75.4 MHz, $\mathrm{CDCl}_{3}$ ): (two isomers) $\delta 147.7$ 
(Cx2), 142.9 (C), 142.3 (C), 129.0 (CHx2), 129.0 (CHx2), 128.2 (CHx2), 128.1 (CHx2), 127.3 (CHx2), 127.0 (CHx2), 126.7 (CH), 126.6 (CH), 116.9 (CHx2), 113.1 (CHx2), 113.1 (CHx2), 62.5 $(\mathrm{CH}), 61.4(\mathrm{CH}), 41.8(\mathrm{CH}), 41.5(\mathrm{CH}), 26.8\left(\mathrm{CH}_{2}\right), 25.3\left(\mathrm{CH}_{2}\right), 16.1\left(\mathrm{CH}_{3}\right), 14.4\left(\mathrm{CH}_{3}\right), 12.0$ $\left(\mathrm{CH}_{3}\right), 11.8\left(\mathrm{CH}_{3}\right)$ ppm; HRMS (EI, m/z): Calcd. for $\mathrm{C}_{17} \mathrm{H}_{21} \mathrm{~N}$ : 239.1674, found $[\mathrm{M}-\mathrm{H}]^{+}$: 238.1592 .

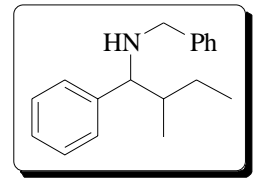

$N$-Benzyl-(1-phenyl-2-methyl-butyl)amine (Table 2, entry 14): Light yellow oil; Yield: 61\%; $\mathrm{R}_{f}$ $=0.38$ (Ethyl acetate/Hexane 1:8); FTIR ( $\mathrm{NaCl}$, neat): $v 3345(\mathrm{NH}) \mathrm{cm}^{-1} ;{ }^{1} \mathrm{H}$ NMR $(300 \mathrm{MHz}$, $\mathrm{CDCl}_{3}$ ): (two isomers) $\delta 0.70-0.93(\mathrm{~m}, 6 \mathrm{H}), 0.95-1.26(\mathrm{~m}, 1 \mathrm{H}), 1.32-1.71(\mathrm{~m}, 2 \mathrm{H}), 1.79(\mathrm{~s}, \mathrm{br}, 1 \mathrm{H})$, 3.43-3.49 (m, 2H), 3.61-3.68 (m, 1H), 7.19-7.35 (m, 10H) ppm; ${ }^{13} \mathrm{C}$ NMR (75.4 MHz, $\mathrm{CDCl}_{3}$ ): (two isomers) $\delta 143.2$ (C), 142.7 (C), 141.0 (C), 140.9 (C), 128.3, 128.2, 128.2, 128.2, 128.0, 128.0, 128.0, 126.7, 126.7 (overall CHx20), $67.0(\mathrm{CH}), 66.9(\mathrm{CH}), 51.7\left(\mathrm{CH}_{2}\right), 51.7\left(\mathrm{CH}_{2}\right), 41.4$ (CH), $40.9(\mathrm{CH}), 26.1\left(\mathrm{CH}_{2}\right), 26.1\left(\mathrm{CH}_{2}\right), 15.6\left(\mathrm{CH}_{3}\right), 15.3\left(\mathrm{CH}_{3}\right), 11.8\left(\mathrm{CH}_{3}\right), 11.4\left(\mathrm{CH}_{3}\right) \mathrm{ppm}$; HRMS (EI, m/z): Calcd. for $\mathrm{C}_{18} \mathrm{H}_{23} \mathrm{~N}$ : 253.1830, found [M-H] $]^{+}: 252.1740$.

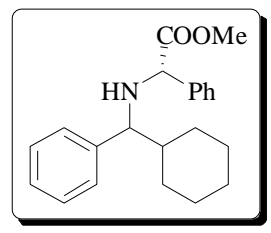

2-[(1-Cyclohexyl-1-phenyl-methyl)-amino]-2-phenyl-acetic acid methyl ester (Table 3, entry 5): Light yellow oil; Yield: 62\%; $\mathrm{R}_{f}=0.55$ and 0.59 (Ethyl acetate/Hexane 1:8); FTIR ( $\mathrm{NaCl}$, neat): $v 3447(\mathrm{NH}), 1738(\mathrm{C}=\mathrm{O}) \mathrm{cm}^{-1} ;{ }^{1} \mathrm{H}$ NMR $\left(400 \mathrm{MHz}, \mathrm{CDCl}_{3}\right)$ : (isomer 1) $\delta$ 0.83-0.96 (m, 1H), 1.05-1.27 (m, 5H), 1.40-1.43 (m, 1H), 1.55-1.62 (m, 3H), 1.75-1.78 (m, 1H), 1.97-2.00 (m, 1H), 3.40 (d, $J=7.08 \mathrm{~Hz}, 1 \mathrm{H}$ ), 3.70 (s, 3H), 4.13 (s, $1 \mathrm{H}$ ), 7.25-7.33 (m, 10H) ppm; (isomer 2) $\delta$ 0.69-0.79 (m, 1H), 0.84-0.94 (m, 1H), 1.02-1.08 (m, 2H), 1.12-1.26 (m, 2H), 1.50-1.56 (m, 3H), 1.69-1.73 (m, 1H), 2.06-2.09 (m, 1H), 2.55 (br, s, 1H), 3.01 (d, $J=7.81 \mathrm{~Hz}, 1 \mathrm{H}), 3.56$ (s, 3H), 4.09 (s, 1H), 7.11-7.13 (m, 2H), 7.22-7.33 (m, 8H) ppm; ${ }^{13} \mathrm{C}$ NMR (100 MHz, $\mathrm{CDCl}_{3}$ ): (isomer 1) $\delta 174.5$ (CO), 142.2 (C), 138.8 (C), 128.6 (CHx2), 128.4 (CHx2), 128.1 (CHx2), 127.8 (CH), 127.1 (CHx3), $67.4(\mathrm{CH}), 62.8(\mathrm{CH}), 51.9\left(\mathrm{CH}_{3}\right), 44.3(\mathrm{CH}), 30.0\left(\mathrm{CH}_{2}\right), 29.7\left(\mathrm{CH}_{2}\right), 26.5\left(\mathrm{CH}_{2}\right)$, $26.2\left(\mathrm{CH}_{2} \mathrm{x} 2\right) \mathrm{ppm}$; (isomer 2) $\delta 173.3(\mathrm{CO}), 142.1(\mathrm{C}), 138.3$ (C), 128.5 (CHx2), 128.1 (CHx4), 128.0 (CHx2), $127.9(\mathrm{CH}), 126.9(\mathrm{CH}), 64.7(\mathrm{CH}), 62.4(\mathrm{CH}), 52.2\left(\mathrm{CH}_{3}\right), 43.9(\mathrm{CH}), 30.2\left(\mathrm{CH}_{2}\right)$, $30.0\left(\mathrm{CH}_{2}\right)$, $26.5\left(\mathrm{CH}_{2}\right), 26.2\left(\mathrm{CH}_{2} \mathrm{x} 2\right)$ ppm; HRMS (EI, m/z): Calcd. for $\mathrm{C}_{22} \mathrm{H}_{27} \mathrm{NO}_{2}$ : 337.2042, found $[\mathrm{M}-\mathrm{H}]^{+}: 336.1946$.

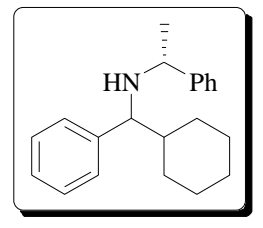


(1-Cyclohexyl-1-phenyl-methyl)-(1-phenyl-ethyl)-amine (Table 3, entry 6): Light yellow oil; Yield: 77\%; $\mathrm{R}_{f}=0.57$ and 0.59 (Ethyl acetate/Hexane 1:8); FTIR ( $\mathrm{NaCl}$, neat): $v 3431(\mathrm{NH}) \mathrm{cm}^{-1}$; ${ }^{1} \mathrm{H}$ NMR (300 MHz, $\mathrm{CDCl}_{3}$ ): (two isomers) $\delta$ 0.71-1.29 (m, 12H, isomer 1 and 2), 1.22 (d, $J=$ $6.66 \mathrm{~Hz}, 3 \mathrm{H}$, isomer 1), 1.28 (d, $J=6.47 \mathrm{~Hz}, 3 \mathrm{H}$, isomer 2), 1.37-1.58 (m, $10 \mathrm{H}$, isomer 1 and 2), 2.01-2.09 (m, 2H, isomer 1 and 2), 3.00 (d, $J=7.87 \mathrm{~Hz}, 1 \mathrm{H}$, isomer 1), 3.41 (q, $\mathrm{J}=6.64 \mathrm{~Hz}, 1 \mathrm{H}$, isomer 1), 3.47 (d, $\mathrm{J}=7.26 \mathrm{~Hz}, 1 \mathrm{H}$, isomer 2) 3.57 (q, $J=6.36 \mathrm{~Hz}, 1 \mathrm{H}$, isomer 2), 7.09-7.32 (m, 20H, isomer 1 and 2) ppm; ${ }^{13} \mathrm{C}$ NMR (75.4 MHz, CDCl $)$ : (two isomers) $\delta 146.7$ (C), 146.0 (C), 143.6 (C), 143.3 (C), 128.2 (CHx2), 128.2 (CHx2), 128.0 (CHx2), 127.9 (CHx2), 127.9 (CHx2), 127.8 (CHx2), 126.8 (CHx2), $126.6(\mathrm{CHx} 4), 126.5$ (CHx2), $65.6(\mathrm{CH}), 65.4(\mathrm{CH}), 54.8(\mathrm{CH})$, $54.5(\mathrm{CH}), 44.3(\mathrm{CH}), 44.2(\mathrm{CH}), 30.6\left(\mathrm{CH}_{2}\right), 30.2\left(\mathrm{CH}_{2}\right), 30.2\left(\mathrm{CH}_{2}\right), 29.9\left(\mathrm{CH}_{2}\right), 26.6\left(\mathrm{CH}_{2}\right)$, $26.6\left(\mathrm{CH}_{2}\right), 26.4\left(\mathrm{CH}_{2}\right), 26.3\left(\mathrm{CH}_{2}\right), 26.3\left(\mathrm{CH}_{2}\right), 26.3\left(\mathrm{CH}_{2}\right), 25.3\left(\mathrm{CH}_{3}\right), 21.9\left(\mathrm{CH}_{3}\right)$ ppm; HRMS (EI, m/z): Calcd. for $\mathrm{C}_{21} \mathrm{H}_{27} \mathrm{~N}$ : 293.2143, found [M-H] ${ }^{+}: 292.2061$.

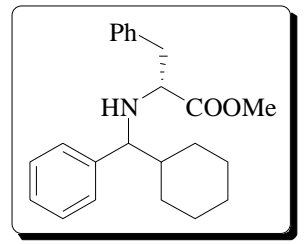

2-[(1-Cyclohexyl-1-phenyl-methyl)-amino]-3-phenyl-propionic acid methyl ester (Table 3, entry 7): Light yellow oil; Yield: $46 \%$; $\mathrm{R}_{f}=0.52$ and 0.55 (Ethyl acetate/Hexane 1:8); FTIR (NaCl, neat): $v 3455(\mathrm{NH}), 1736(\mathrm{C}=\mathrm{O}) \mathrm{cm}^{-1} ;{ }^{1} \mathrm{H}$ NMR (300 $\mathrm{MHz}, \mathrm{CDCl}_{3}$ ): (two isomers) $\delta$ 0.68-1.26 (m, 10H, isomer 1 and 2), 1.34-1.72 (m, 10H, isomer 1 and 2), 1.81-1.96 (m, $4 \mathrm{H}$, isomer1 and 2), 2.75-2.90 (m, 4H, isomer 1 and 2), 3.18-3.40 (m, 4H, isomer 1 and 2), 3.36 (s, 3H, isomer 1), 3.61 (s, 3H, isomer 2), 6.98-7.28 (m, 20H, isomer 1 and 2) ppm; ${ }^{13} \mathrm{C}$ NMR (75.4 MHz, $\mathrm{CDCl}_{3}$ ): (two isomers) $\delta 174.8$ (CO), 175.7 (CO), 142.4 (C), 142.0 (C), 137.6 (C), 137.5 (C), 129.3 (CHx2), 129.3 (CHx2), 128.2 (CHx2), 128.2 (CHx2), 128.1 (CHx2), 128.1 (CHx2), 127.9 (CHx2), 127.7 (CHx2), $126.9(\mathrm{CH}), 126.7(\mathrm{CH}), 126.5(\mathrm{CH}), 126.4(\mathrm{CH}), 67.7(\mathrm{CH}), 66.9(\mathrm{CH})$, $61.3(\mathrm{CH}), 60.2(\mathrm{CH}), 51.4\left(\mathrm{CH}_{3}\right), 51.4\left(\mathrm{CH}_{3}\right), 44.4(\mathrm{CH}), 43.8(\mathrm{CH}), 40.2\left(\mathrm{CH}_{2}\right), 39.2\left(\mathrm{CH}_{2}\right), 30.3$ $\left(\mathrm{CH}_{2}\right), 29.8\left(\mathrm{CH}_{2}\right), 29.6\left(\mathrm{CH}_{2}\right), 29.5\left(\mathrm{CH}_{2}\right), 26.4\left(\mathrm{CH}_{2} \mathrm{x} 2\right), 26.2\left(\mathrm{CH}_{2}\right), 26.2\left(\mathrm{CH}_{2} \mathrm{x} 2\right), 26.1\left(\mathrm{CH}_{2}\right)$ ppm; HRMS (EI, m/z): Calcd. for $\mathrm{C}_{23} \mathrm{H}_{29} \mathrm{NO}_{2}$ : 351.2198, found [M-H] $]^{+}$: 350.2109 .

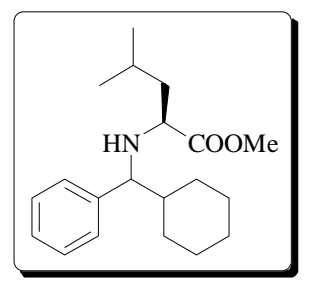

2-[(1-Cyclohexyl-1-phenyl-methyl)-amino]-4-methyl-pentanoic acid methyl ester (Table 3, entry 8): Colorless oil; Yield: 72\%; $\mathrm{R}_{f}=0.60$ and 0.70 (Ethyl acetate/Hexane 1:8); FTIR (NaCl, neat): $v 3455(\mathrm{NH}), 1736(\mathrm{C}=\mathrm{O}) \mathrm{cm}^{-1}$; ${ }^{1} \mathrm{H}$ NMR $\left(300 \mathrm{MHz}, \mathrm{CDCl}_{3}\right)$ : (major isomer) $\delta 0.64$ (d, $J=$ $6.57 \mathrm{~Hz}, 3 \mathrm{H}), 0.83$ (d, $J=6.69 \mathrm{~Hz}, 3 \mathrm{H}), 0.85-0.90(\mathrm{~m}, 1 \mathrm{H}), 0.97-1.59(\mathrm{~m}, 10 \mathrm{H}), 1.73-1.95(\mathrm{~m}, 4 \mathrm{H})$, 2.94-2.99 (m, 1H), 3.20 (d, $J=7.11 \mathrm{~Hz}, 1 \mathrm{H}), 3.68$ (s, 3H), 7.11-7.30 (m, 5H) ppm; ${ }^{13} \mathrm{C}$ NMR $(75.4$ $\mathrm{MHz}, \mathrm{CDCl}_{3}$ ): (major isomer) $\delta 177.2$ (CO), 142.5 (C), 128.4 (CHx2), 127.8 (CHx2), $126.9(\mathrm{CH})$, $67.2(\mathrm{CH}), 57.2(\mathrm{CH}), 51.4\left(\mathrm{CH}_{3}\right), 44.3(\mathrm{CH}), 43.4\left(\mathrm{CH}_{2}\right), 29.9\left(\mathrm{CH}_{2}\right), 29.7\left(\mathrm{CH}_{2}\right), 26.5\left(\mathrm{CH}_{2}\right)$, 
$26.2\left(\mathrm{CH}_{2}\right), 26.2\left(\mathrm{CH}_{2}\right), 24.6\left(\mathrm{CH}_{3}\right), 23.1(\mathrm{CH}), 21.6\left(\mathrm{CH}_{3}\right)$ ppm; HRMS (EI, m/z): Calcd. for $\mathrm{C}_{20} \mathrm{H}_{31} \mathrm{NO}_{2}$ : 317.2355, found [M-H] $]^{+}$316.2280.

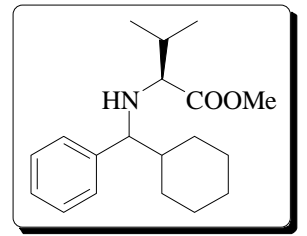

2-[(1-Cyclohexyl-1-phenyl-methyl)-amino]-3-methyl-butyric acid methyl ester (Table 4, entry 1): Colorless oil; Yield: $77 \% ; \mathrm{R}_{f}=0.65$ (Ethyl acetate/Hexane 1:8); FTIR (NaCl, neat): $v$ $3445(\mathrm{NH}), 1732(\mathrm{C}=\mathrm{O}) \mathrm{cm}^{-1}$; ${ }^{1} \mathrm{H}$ NMR (400 MHz, $\mathrm{CDCl}_{3}$ ): (major isomer) $\delta 0.83(\mathrm{~d}, J=6.73 \mathrm{~Hz}$, $3 \mathrm{H}), 0.90$ (d, $J=6.81 \mathrm{~Hz}, 3 \mathrm{H}), 0.83-1.01(\mathrm{~m}, 2 \mathrm{H}), 1.04-1.12(\mathrm{~m}, 2 \mathrm{H}), 1.17-1.29(\mathrm{~m}, 1 \mathrm{H})$, 1.36-1.39 (m, 1H), 1.45-1.52 (m, 1H), 1.60-1.61 (m, 2H), 1.72-1.83 (m, 2H), 1.89-1.94 (m, 2H), 2.71 (d, $J=6.32 \mathrm{~Hz}, 1 \mathrm{H}), 3.17$ (d, $J=6.92 \mathrm{~Hz}, 1 \mathrm{H}), 3.69$ (s, 3H), 7.13-7.29 (m, 5H) ppm; ${ }^{13} \mathrm{C}$ NMR (100 MHz, $\mathrm{CDCl}_{3}$ ): (major isomer) $\delta 176.4$ (CO), 142.5 (C), 128.6 (CHx2), 127.7 (CHx2), $126.8(\mathrm{CH}), 67.3(\mathrm{CH}), 64.5(\mathrm{CH}), 51.2\left(\mathrm{CH}_{3}\right), 44.4(\mathrm{CH}), 31.8(\mathrm{CH}), 29.9\left(\mathrm{CH}_{2}\right), 29.7\left(\mathrm{CH}_{2}\right)$, $26.5\left(\mathrm{CH}_{2}\right), 26.2\left(\mathrm{CH}_{2}\right), 26.2\left(\mathrm{CH}_{2}\right), 19.5\left(\mathrm{CH}_{3}\right), 18.6\left(\mathrm{CH}_{3}\right) \mathrm{ppm}$; HRMS (EI, m/z): Calcd. for $\mathrm{C}_{19} \mathrm{H}_{29} \mathrm{NO}_{2}$ : 303.2198, found $[\mathrm{M}+\mathrm{H}]^{+}:$304.2245.

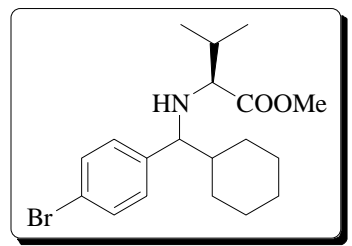

2-\{[1-Cyclohexyl-1-(p-bromophenyl)-methyl]-amino\}-3-methyl-butyric acid methyl ester (Table 4, entry 2): Colorless oil; Yield: 63\%; $\mathrm{R}_{f}=0.61$ (Ethyl acetate/Hexane 1:8); FTIR ( $\mathrm{NaCl}$, neat): $v 3447(\mathrm{NH}), 1734(\mathrm{C}=\mathrm{O}) \mathrm{cm}^{-1}$; ${ }^{1} \mathrm{H} \mathrm{NMR}$ (300 MHz, $\left.\mathrm{CDCl}_{3}\right)$ : (major isomer) $\delta 0.84(\mathrm{~d}, J=$ $6.76 \mathrm{~Hz}, 3 \mathrm{H}), 0.90$ (d, $J=6.78 \mathrm{~Hz}, 3 \mathrm{H}), 0.98-1.92$ (m, 12H), 2.69 (d, $J=6.28 \mathrm{~Hz}, 1 \mathrm{H}), 2.87$ (s, br, 1H), 3.17 (d, $J=6.97 \mathrm{~Hz}, 1 \mathrm{H}), 3.70$ (s, 3H), 7.13 (d, $J=8.22 \mathrm{~Hz}, 2 \mathrm{H}), 7.40$ (d, $J=8.22 \mathrm{~Hz}, 2 \mathrm{H})$ ppm; ${ }^{13} \mathrm{C}$ NMR (75.4 MHz, $\mathrm{CDCl}_{3}$ ): (major isomer) $\delta 175.7$ (CO), 141.0 (C), 131.0 (CHx2), 130.3 (CHx2), $120.7(\mathrm{C}), 66.9(\mathrm{CH}), 64.6(\mathrm{CH}), 51.4\left(\mathrm{CH}_{3}\right), 44.1(\mathrm{CH}), 31.6(\mathrm{CH}), 29.9\left(\mathrm{CH}_{2}\right), 29.5$ $\left(\mathrm{CH}_{2}\right)$, $26.4\left(\mathrm{CH}_{2}\right), 26.1\left(\mathrm{CH}_{2} \mathrm{x} 2\right)$, $19.4\left(\mathrm{CH}_{3}\right)$, $18.6\left(\mathrm{CH}_{3}\right) \mathrm{ppm}$; HRMS (EI, m/z): Calcd. for $\mathrm{C}_{19} \mathrm{H}_{28} \mathrm{BrNO}_{2}$ : 381.1303, found $[\mathrm{M}-\mathrm{H}]^{+}: 380.1224$.

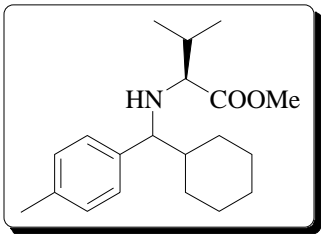

2-\{[1-Cyclohexyl-1-(p-methylphenyl)-methyl]-amino\}-3-methyl-butyric acid methyl ester (Table 4, entry 3): Colorless oil; Yield: 80\%; $\mathrm{R}_{f}=0.61$ (Ethyl acetate/Hexane 1:8); FTIR ( $\mathrm{NaCl}$, neat): $v 3447(\mathrm{NH}), 1734(\mathrm{C}=\mathrm{O}) \mathrm{cm}^{-1}$; ${ }^{1} \mathrm{H} \mathrm{NMR}$ (400 MHz, $\left.\mathrm{CDCl}_{3}\right)$ : (major isomer) $\delta 0.83(\mathrm{~d}, J=$ $6.68 \mathrm{~Hz}, 3 \mathrm{H}), 0.90$ (d, $J=6.76 \mathrm{~Hz}, 3 \mathrm{H}), 0.94-1.93(\mathrm{~m}, 13 \mathrm{H}), 2.32$ (s, 3H), 2.72 (d, $J=6.40 \mathrm{~Hz}$, 1H), 3.14 (d, $J=6.88 \mathrm{~Hz}, 1 \mathrm{H}), 3.68$ (s, 3H), 7.06-7.14 (m, 4H) ppm; $\left.{ }^{13} \mathrm{C} \mathrm{NMR} \mathrm{(100} \mathrm{MHz,} \mathrm{CDCl}\right)_{3}$ : 
(major isomer) $\delta 176.4(\mathrm{CO}), 139.4(\mathrm{C}), 136.2(\mathrm{C}), 128.4(\mathrm{CHx} 4), 66.9(\mathrm{CH}), 64.5(\mathrm{CH}), 51.1$ $\left(\mathrm{CH}_{3}\right), 44.5(\mathrm{CH}), 31.8(\mathrm{CH}), 29.9\left(\mathrm{CH}_{2}\right), 29.7\left(\mathrm{CH}_{2}\right), 26.5\left(\mathrm{CH}_{2}\right), 26.2\left(\mathrm{CH}_{2}\right), 26.2\left(\mathrm{CH}_{2}\right), 21.1$ $\left(\mathrm{CH}_{3}\right), 19.4\left(\mathrm{CH}_{3}\right), 18.6\left(\mathrm{CH}_{3}\right)$ ppm; HRMS (EI, m/z): Calcd. for $\mathrm{C}_{20} \mathrm{H}_{31} \mathrm{NO}_{2}$ : 317.2355, found $[\mathrm{M}-\mathrm{H}]^{+}: 316.2272$.

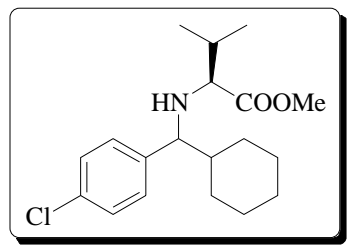

2-\{[1-Cyclohexyl-1-(p-chlorophenyl)-methyl]-amino\}-3-methyl-butyric acid methyl ester (Table 4, entry 4): Colorless oil; Yield: 58\%; $\mathrm{R}_{f}=0.63$ (Ethyl acetate/Hexane 1:8); FTIR (NaCl, neat): $v 3447(\mathrm{NH}), 1732(\mathrm{C}=\mathrm{O}) \mathrm{cm}^{-1}$; ${ }^{1} \mathrm{H} \mathrm{NMR}$ (300 MHz, $\mathrm{CDCl}_{3}$ ): (major isomer) $\delta 0.83(\mathrm{~d}, J=$ $6.75 \mathrm{~Hz}, 3 \mathrm{H}), 0.89$ (d, $J=6.81 \mathrm{~Hz}, 3 \mathrm{H}), 0.95-1.90$ (m, 13H), 2.66 (d, $J=6.40 \mathrm{~Hz}, 1 \mathrm{H}), 3.16$ (d, $J$ $=6.75 \mathrm{~Hz}, 1 \mathrm{H}), 3.70(\mathrm{~s}, 3 \mathrm{H}), 7.17-7.26(\mathrm{~m}, 4 \mathrm{H}) \mathrm{ppm} ;{ }^{13} \mathrm{C} \mathrm{NMR}\left(75.4 \mathrm{MHz}, \mathrm{CDCl}_{3}\right)$ : (major isomer) $\delta 176.2$ (CO), $141.1(\mathrm{C}), 132.4(\mathrm{C}), 129.9$ (CHx2), 127.9 (CHx2), $66.7(\mathrm{CH}), 64.6(\mathrm{CH})$, $51.3\left(\mathrm{CH}_{3}\right), 44.4(\mathrm{CH}), 31.8(\mathrm{CH}), 29.9\left(\mathrm{CH}_{2}\right), 29.5\left(\mathrm{CH}_{2}\right), 26.4\left(\mathrm{CH}_{2}\right), 26.2\left(\mathrm{CH}_{2} \mathrm{x}\right), 19.5\left(\mathrm{CH}_{3}\right)$, $18.5\left(\mathrm{CH}_{3}\right)$ ppm; HRMS (EI, m/z): Calcd. for $\mathrm{C}_{19} \mathrm{H}_{28} \mathrm{ClNO}_{2}$ : 337.1809, found $[\mathrm{M}-\mathrm{H}]^{+}: 336.1722$

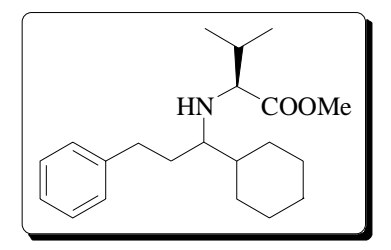

2-[(1-Cyclohexyl-3-phenyl-propyl)-amino]-3-methyl-butyric acid methyl ester (Table 4, entry 5): Colorless oil; Yield: $81 \% ; \mathrm{R}_{f}=0.30$ and 0.39 (Ethyl acetate/Hexane 1:8); FTIR ( $\mathrm{NaCl}$, neat): $v 3449(\mathrm{NH}), 1736(\mathrm{C}=\mathrm{O}) \mathrm{cm}^{-1}$; ${ }^{1} \mathrm{H}$ NMR $\left(300 \mathrm{MHz}, \mathrm{CDCl}_{3}\right.$ ): (major isomer) $\delta 0.86$ (d, $J=6.66$ $\mathrm{Hz}, 3 \mathrm{H}$ ), 0.90 (d, $J=6.79 \mathrm{~Hz}, 3 \mathrm{H}), 0.94-1.35$ (m, $5 \mathrm{H}), 1.44-1.87$ (m, 10H), 2.13 (q, $J=5.12 \mathrm{~Hz}$, $1 \mathrm{H}$ ), 2.58 (t, $J=8.25 \mathrm{~Hz}, 2 \mathrm{H}), 2.92$ (d, $J=6.54 \mathrm{~Hz}, 1 \mathrm{H}), 3.59$ (s, 3H), 7.04-7.20 (m, 5H) ppm; ${ }^{13} \mathrm{C}$ NMR (75.4 MHz, $\mathrm{CDCl}_{3}$ ): (major isomer) $\delta 176.4$ (CO), 143.1 (C), 128.3 (CHx2), 128.2 (CHx2), $125.5(\mathrm{CH}), 65.5(\mathrm{CH}), 61.3(\mathrm{CH}), 51.2\left(\mathrm{CH}_{3}\right), 40.5(\mathrm{CH}), 32.6\left(\mathrm{CH}_{2}\right), 32.2(\mathrm{CH}), 31.7\left(\mathrm{CH}_{2}\right)$, $29.3\left(\mathrm{CH}_{2}\right)$, $28.6\left(\mathrm{CH}_{2}\right), 26.6\left(\mathrm{CH}_{2}\right), 26.6\left(\mathrm{CH}_{2}\right), 26.5\left(\mathrm{CH}_{2}\right), 19.4\left(\mathrm{CH}_{3}\right), 18.9\left(\mathrm{CH}_{3}\right)$ ppm; HRMS (EI, m/z): Calcd. for $\mathrm{C}_{21} \mathrm{H}_{33} \mathrm{NO}_{2}$ : 331.2511, found [M-H] $]^{+}$: 330.2411 .

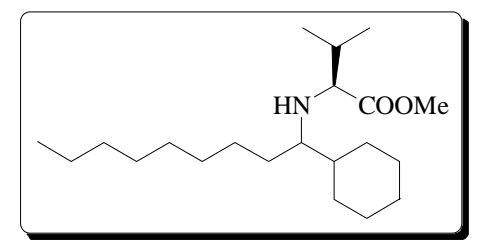

2-[(1-Cyclohexyl-nonyl)-amino]-3-methyl-butyric acid methyl ester (Table 4, entry 6): Colorless oil; Yield: 66\%; $\mathrm{R}_{f}=0.53$ and 0.62 (Ethyl acetate/Hexane 1:8); FTIR (NaCl, neat): $v$ $3455(\mathrm{NH}), 1736(\mathrm{C}=\mathrm{O}) \mathrm{cm}^{-1}$; ${ }^{1} \mathrm{H}$ NMR (300 $\left.\mathrm{MHz}, \mathrm{CDCl}_{3}\right)$ : (major isomer) $\delta$ 0.86-0.96 (m, 9H), 1.16-1.29 (m, 21H), 1.58-1.85 (m, 6H), 2.11-2.12 (m, 1H), 2.99 (d, $J=6.60 \mathrm{~Hz}, 1 \mathrm{H}), 3.69$ (s, 3H) ppm; ${ }^{13} \mathrm{C}$ NMR (75.4 MHz, $\mathrm{CDCl}_{3}$ ): (major isomer) $\delta 176.5(\mathrm{CO}), 65.6(\mathrm{CH}), 61.6(\mathrm{CH}), 51.2$ 
$\left(\mathrm{CH}_{3}\right), 40.7(\mathrm{CH}), 32.1(\mathrm{CH}), 31.9\left(\mathrm{CH}_{2}\right), 30.6\left(\mathrm{CH}_{2}\right), 30.1\left(\mathrm{CH}_{2}\right), 29.6\left(\mathrm{CH}_{2}\right), 29.3\left(\mathrm{CH}_{2}\right), 29.0$

$\left(\mathrm{CH}_{2}\right)$, $28.9\left(\mathrm{CH}_{2}\right), 26.7\left(\mathrm{CH}_{2}\right), 26.7\left(\mathrm{CH}_{2}\right), 26.6\left(\mathrm{CH}_{2}\right), 25.6\left(\mathrm{CH}_{2}\right), 22.7\left(\mathrm{CH}_{2}\right), 19.3\left(\mathrm{CH}_{3}\right), 18.9$

$\left(\mathrm{CH}_{3}\right)$, $14.1\left(\mathrm{CH}_{3}\right)$ ppm; HRMS (EI, m/z): Calcd. for $\mathrm{C}_{21} \mathrm{H}_{41} \mathrm{NO}_{2}$ : 339.3137, found [M-H] $]^{+}$: 338.3055 .

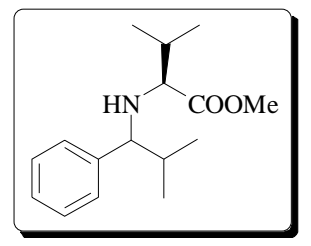

2-[(2-Methyl-1-phenyl-propyl)-amino]-3-methyl-butyric acid methyl ester (Table 4, entry 7): Colorless oil; Yield: 52\%; $\mathrm{R}_{f}=0.61$ (Ethyl acetate/Hexane 1:8); FTIR ( $\mathrm{NaCl}$, neat): $v 3443(\mathrm{NH})$, $1732(\mathrm{C}=\mathrm{O}) \mathrm{cm}^{-1}$; ${ }^{1} \mathrm{H}$ NMR (300 MHz, $\mathrm{CDCl}_{3}$ ): (major isomer) $\delta 0.73(\mathrm{~d}, J=6.81 \mathrm{~Hz}, 3 \mathrm{H}), 0.84$ (d, $J=6.78 \mathrm{~Hz}, 3 \mathrm{H}), 0.91$ (d, $J=6.81 \mathrm{~Hz}, 3 \mathrm{H}), 0.96$ (d, $J=6.69 \mathrm{~Hz}, 3 \mathrm{H}), 1.75-1.87$ (m, 2H), 1.90 (br, s, 1H), 2.72 (d, $J=6.39 \mathrm{~Hz}, 1 \mathrm{H}$ ), 3.17 (d, $J=6.73 \mathrm{~Hz}, 1 \mathrm{H}$ ), 3.69 (s, 3H), 7.13-7.30 (m, 5H) ppm; ${ }^{13} \mathrm{C}$ NMR (75.4 MHz, $\mathrm{CDCl}_{3}$ ): (major isomer) $\delta 175.8$ (C), 141.7 (C), 128.6 (CHx2), 127.9 (CHx2), $127.1(\mathrm{CH}), 68.2(\mathrm{CH}), 64.6(\mathrm{CH}), 51.3\left(\mathrm{CH}_{3}\right), 34.5(\mathrm{CH}), 31.6(\mathrm{CH}), 19.4\left(\mathrm{CH}_{3}\right), 19.3$ $\left(\mathrm{CH}_{3} \mathrm{x} 2\right), 18.7\left(\mathrm{CH}_{3}\right) \mathrm{ppm}$; HRMS (EI, m/z): Calcd. for $\mathrm{C}_{16} \mathrm{H}_{25} \mathrm{NO}_{2}$ : 263.1885, found $[\mathrm{M}-\mathrm{H}]^{+}$: 262.1805 .

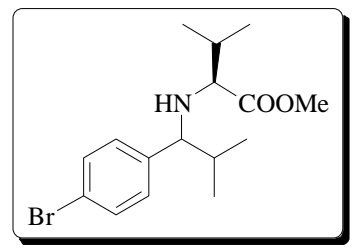

2-\{[2-Methyl-1-(p-bromophenyl)-propyl]-amino\}-3-methyl-butyric acid methyl ester (Table 4, entry 8): Colorless oil; Yield: $50 \% ; \mathrm{R}_{f}=0.46$ (Ethyl acetate/Hexane 1:8); FTIR ( $\mathrm{NaCl}$, neat): $v$ $3449(\mathrm{NH}), 1736(\mathrm{C}=\mathrm{O}) \mathrm{cm}^{-1}$; ${ }^{1} \mathrm{H}$ NMR (300 MHz, $\mathrm{CDCl}_{3}$ ): (major isomer) $\delta 0.72(\mathrm{~d}, J=6.84 \mathrm{~Hz}$, $3 \mathrm{H}), 0.84$ (d, $J=6.76 \mathrm{~Hz}, 3 \mathrm{H}), 0.90$ (d, $J=6.84 \mathrm{~Hz}, 3 \mathrm{H}), 0.94$ (d, $J=6.70 \mathrm{~Hz}, 3 \mathrm{H}), 1.75-1.87$ (m, 3H), 2.66 (d, $J=6.45 \mathrm{~Hz}, 1 \mathrm{H}$ ), 3.14 (d, $J=6.60 \mathrm{~Hz}, 1 \mathrm{H}$ ), 3.70 (s, 3H), 7.15 (d, $J=8.38 \mathrm{~Hz}, 2 \mathrm{H}$ ), 7.41 (d, $J=8.38 \mathrm{~Hz}, 2 \mathrm{H}) \mathrm{ppm} ;{ }^{13} \mathrm{C} \mathrm{NMR}\left(75.4 \mathrm{MHz}, \mathrm{CDCl}_{3}\right.$ ): (major isomer) $\delta 176.1$ (CO), 141.4 (C), 130.9 (CHx2), 130.3 (CHx2), $120.6(\mathrm{C}), 67.3(\mathrm{CH}), 64.6(\mathrm{CH}), 51.3\left(\mathrm{CH}_{3}\right), 34.7(\mathrm{CH}), 31.8$ (CH), $19.5\left(\mathrm{CH}_{3}\right), 19.3\left(\mathrm{CH}_{3}\right), 19.1\left(\mathrm{CH}_{3}\right), 18.5\left(\mathrm{CH}_{3}\right)$ ppm; HRMS (EI, m/z): Calcd. for $\mathrm{C}_{16} \mathrm{H}_{24} \mathrm{BrNO}_{2}$ : 341.0990, found [M-H] $]^{+}$340.0906.

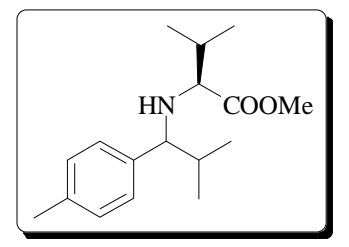

2-\{[2-Methyl-1-(p-methylphenyl)-propyl]-amino\}-3-methyl-butyric acid methyl ester (Table 4, entry 9): Colorless oil; Yield: 66\%; $\mathrm{R}_{f}=0.59$ (Ethyl acetate/Hexane 1:8); FTIR ( $\mathrm{NaCl}$, neat): $v$ $3447(\mathrm{NH}), 1734(\mathrm{C}=\mathrm{O}) \mathrm{cm}^{-1} ;{ }^{1} \mathrm{H} \mathrm{NMR}\left(300 \mathrm{MHz}, \mathrm{CDCl}_{3}\right.$ ): (major isomer) $\delta 0.73$ (d, $J=6.84 \mathrm{~Hz}$, 3H), 0.84 (d, $J=6.79 \mathrm{~Hz}, 3 \mathrm{H}), 0.91$ (d, $J=6.82 \mathrm{~Hz}, 3 \mathrm{H}), 0.95$ (d, $J=6.66 \mathrm{~Hz}, 3 \mathrm{H}), 1.74-1.92$ (m, 
3H), 2.32 (s, 3H), 2.73 (d, $J=6.42 \mathrm{~Hz}, 1 \mathrm{H}), 3.13$ (d, $J=6.72 \mathrm{~Hz}, 1 \mathrm{H}), 3.69$ (s, 3H), 7.06-7.6 (m, 4H) ppm; ${ }^{13} \mathrm{C}$ NMR (75.4 MHz, $\mathrm{CDCl}_{3}$ ): (major isomer) $\delta 176.4$ (CO), 139.2 (C), 136.3 (C), 128.5 (CHx4), $67.6(\mathrm{CH}), 64.5(\mathrm{CH}), 51.1\left(\mathrm{CH}_{3}\right), 34.8(\mathrm{CH}), 31.8(\mathrm{CH}), 21.1\left(\mathrm{CH}_{3}\right), 19.5\left(\mathrm{CH}_{3}\right), 19.4$ $\left(\mathrm{CH}_{3}\right), 19.2\left(\mathrm{CH}_{3}\right), 18.6\left(\mathrm{CH}_{3}\right)$ ppm; HRMS (EI, m/z): Calcd. for $\mathrm{C}_{17} \mathrm{H}_{27} \mathrm{NO}_{2}$ : 277.2042, found $[\mathrm{M}-\mathrm{H}]^{+}: 276.1952$.

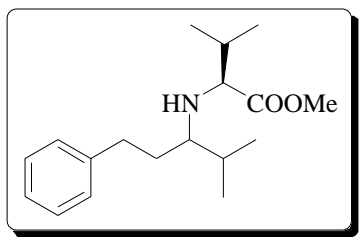

3-Methyl-2-[(2-methyl-1-phenethyl-propyl)amino]-butyric acid methyl ester (Table 4, entry 10): Colorless oil; Yield: $72 \% ; \mathrm{R}_{f}=0.43$ and 0.55 (Ethyl acetate/Hexane 1:8); FTIR ( $\mathrm{NaCl}$, neat): $v 3449(\mathrm{NH}), 1736(\mathrm{C}=\mathrm{O}) \mathrm{cm}^{-1}$; ${ }^{1} \mathrm{H}$ NMR (300 MHz, $\mathrm{CDCl}_{3}$ ): (major isomer) $\delta 0.88$ (d, $J=3.78$ Hz, 3H), 0.90 (d, $J=3.78 \mathrm{~Hz}, 3 \mathrm{H}), 0.94$ (d, $J=6.78 \mathrm{~Hz}, 3 \mathrm{H}), 0.98$ (d, $J=6.76 \mathrm{~Hz}, 3 \mathrm{H}), 1.49-1.96$ (m, 5H), 2.21 (q, $J=5.25 \mathrm{~Hz}, 1 \mathrm{H}), 2.67(\mathrm{t}, J=8.35 \mathrm{~Hz}, 2 \mathrm{H}), 3.01$ (d, $J=6.51 \mathrm{~Hz}, 1 \mathrm{H}$ ), 3.67 (s, 3H), 7.13-7.29 (m, 5H) ppm; ${ }^{13} \mathrm{C}$ NMR (75.4 MHz, $\mathrm{CDCl}_{3}$ ): (major isomer) $\delta 176.4$ (CO), 143.1 (C), 128.3 (CHx2), $128.3(\mathrm{CHx} 2), 125.6(\mathrm{CH}), 65.5(\mathrm{CH}), 62.0(\mathrm{CH}), 51.2\left(\mathrm{CH}_{3}\right), 32.5\left(\mathrm{CH}_{2}\right)$, $32.3(\mathrm{CH}), 31.9\left(\mathrm{CH}_{2}\right), 30.1(\mathrm{CH}), 19.4\left(\mathrm{CH}_{3}\right), 18.9\left(\mathrm{CH}_{3}\right), 18.7\left(\mathrm{CH}_{3}\right), 18.1\left(\mathrm{CH}_{3}\right) \mathrm{ppm}$; HRMS (EI, m/z): Calcd. for $\mathrm{C}_{18} \mathrm{H}_{29} \mathrm{NO}_{2}$ : 291.2198, found [M-H] $]^{+}: 290.2117$.

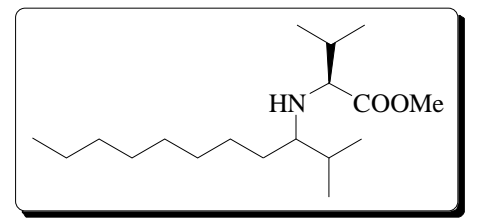

2-[(1-Isopropyl-nonyl)-amino]-3-methyl-butyric acid methyl ester (Table 4, entry 11): Colorless oil; Yield: $72 \% ; \mathrm{R}_{f}=0.5$ and 0.58 (Ethyl acetate/Hexane 1:8); FTIR ( $\mathrm{NaCl}$, neat): $v$ $3455(\mathrm{NH}), 1738(\mathrm{C}=\mathrm{O}) \mathrm{cm}^{-1}$; ${ }^{1} \mathrm{H}$ NMR (400 MHz, $\left.\mathrm{CDCl}_{3}\right)$ : (major isomer) $\delta$ 0.85-0.97 (m, 15H), 1.27-1.42 (m, 15H), 1.64-1.71 (m, 1H), 1.79-1.87 (m, 1H), 2.13-2.14 (m, $1 \mathrm{H}), 3.00$ (d, $J=6.60 \mathrm{~Hz}$, $1 \mathrm{H}), 3.70(\mathrm{~s}, 3 \mathrm{H}) \mathrm{ppm} ;{ }^{13} \mathrm{C} \mathrm{NMR}\left(100 \mathrm{MHz}, \mathrm{CDCl}_{3}\right)$ : (major isomer) $\delta 176.4(\mathrm{CO}), 65.6(\mathrm{CH})$, $62.1(\mathrm{CH}), 51.2\left(\mathrm{CH}_{3}\right), 32.1(\mathrm{CH}), 31.9\left(\mathrm{CH}_{2}\right), 30.5\left(\mathrm{CH}_{2}\right), 30.2(\mathrm{CH}), 30.1\left(\mathrm{CH}_{2}\right), 29.6\left(\mathrm{CH}_{2}\right)$, $29.3\left(\mathrm{CH}_{2}\right), 25.7\left(\mathrm{CH}_{2}\right), 22.7\left(\mathrm{CH}_{2}\right), 19.3\left(\mathrm{CH}_{3}\right), 18.9\left(\mathrm{CH}_{3}\right), 18.3\left(\mathrm{CH}_{3} \mathrm{x}\right), 14.1\left(\mathrm{CH}_{3}\right) \mathrm{ppm}$; HRMS (EI, m/z): Calcd. for $\mathrm{C}_{18} \mathrm{H}_{37} \mathrm{NO}_{2}$ : 299.2824, found [M-H] $]^{+}: 298.2738$.

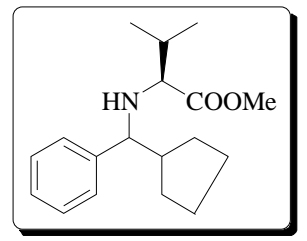

2-[(1-Cyclopentyl-1-phenyl-methyl)-amino]-3-methyl-butyric acid methyl ester (Table 4, entry 12): Colorless oil; Yield: $54 \% ; \mathrm{R}_{f}=0.61$ (Ethyl acetate/Hexane 1:8); FTIR ( $\mathrm{NaCl}$, neat): $v$ $3447(\mathrm{NH}), 1734(\mathrm{C}=\mathrm{O}) \mathrm{cm}^{-1}$; ${ }^{1} \mathrm{H}$ NMR (300 MHz, $\left.\mathrm{CDCl}_{3}\right)$ : (major isomer) $\delta 0.81(\mathrm{~d}, J=6.74 \mathrm{~Hz}$, $3 \mathrm{H}), 0.88(\mathrm{~d}, J=6.81 \mathrm{~Hz}, 3 \mathrm{H}), 1.02-1.12(\mathrm{~m}, 1 \mathrm{H}), 1.18-1.24(\mathrm{~m}, 1 \mathrm{H}), 1.29-1.46(\mathrm{~m}, 2 \mathrm{H})$, 
1.50-1.64 (m, 3H), 1.72-1.83 (m, 1H), 1.88-2.07 (m, 3H), 2.70 (d, $J=6.30 \mathrm{~Hz}, 1 \mathrm{H}), 3.14$ (d, $J=$ $8.74 \mathrm{~Hz}, 1 \mathrm{H}), 3.70$ (s, 3H), 7.20-7.28 (m, 5H) ppm; ${ }^{13} \mathrm{C} \mathrm{NMR}$ (75.4 MHz, $\mathrm{CDCl}_{3}$ ): (major isomer) $\delta 176.3$ (CO), 143.6 (C), 128.2 (CHx2), 127.9 (CHx2), $126.9(\mathrm{CH}), 67.6(\mathrm{CH}), 64.4(\mathrm{CH}), 51.2$ $\left(\mathrm{CH}_{3}\right)$, $47.6(\mathrm{CH}), 31.7(\mathrm{CH}), 30.2\left(\mathrm{CH}_{2}\right), 30.0\left(\mathrm{CH}_{2}\right), 25.4\left(\mathrm{CH}_{2}\right), 25.1\left(\mathrm{CH}_{2}\right), 19.4\left(\mathrm{CH}_{3}\right), 18.5$ $\left(\mathrm{CH}_{3}\right)$ ppm; HRMS (EI, m/z): Calcd. for $\mathrm{C}_{18} \mathrm{H}_{27} \mathrm{NO}_{2}$ : 289.2042, found [M-H] $]^{+}: 288.1964$.

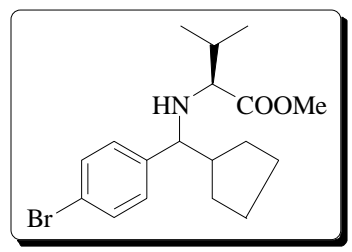

2-\{[1-Cyclopentyl-1-(p-bromophenyl)-methyl]-amino\}-3-methyl-butyric acid methyl ester (Table 4, entry 13): Colorless oil; Yield: 57\%; $\mathrm{R}_{f}=0.5$ (Ethyl acetate/Hexane 1:8); FTIR ( $\mathrm{NaCl}$, neat): $v 3451(\mathrm{NH}), 1732(\mathrm{C}=\mathrm{O}) \mathrm{cm}^{-1}$; ${ }^{1} \mathrm{H} \mathrm{NMR}\left(400 \mathrm{MHz}, \mathrm{CDCl}_{3}\right)$ : (major isomer) $\delta 0.82$ (d, $J=$ $6.77 \mathrm{~Hz}, 3 \mathrm{H}), 0.87$ (d, $J=6.80 \mathrm{~Hz}, 3 \mathrm{H}), 0.98-1.07$ (m, $1 \mathrm{H}), 1.19-1.26(\mathrm{~m}, 1 \mathrm{H}), 1.33-1.46(\mathrm{~m}, 2 \mathrm{H})$, 1.51-1.67 (m, 4H), 1.74-1.85 (m, 1H), 1.88-2.03 (m, 2H), 2.64 (d, $J=6.32 \mathrm{~Hz}, 1 \mathrm{H}), 3.10$ (d, $J=$ $8.60 \mathrm{~Hz}, 1 \mathrm{H}), 3.71$ (s, 3H), 7.17 (d, $J=8.36 \mathrm{~Hz}, 2 \mathrm{H}), 7.40$ (d, $J=8.36 \mathrm{~Hz}, 2 \mathrm{H}) \mathrm{ppm} ;{ }^{13} \mathrm{C}$ NMR (100 MHz, $\mathrm{CDCl}_{3}$ ): (major isomer) $\delta 176.1$ (CO), 142.8 (C), 131.1 (CHx2), 129.9 (CHx2), 120.5 (C), $67.0(\mathrm{CH}), 64.4(\mathrm{CH}), 51.3\left(\mathrm{CH}_{3}\right), 47.5(\mathrm{CH}), 31.7(\mathrm{CH}), 30.1\left(\mathrm{CH}_{2}\right), 29.9\left(\mathrm{CH}_{2}\right), 25.3\left(\mathrm{CH}_{2}\right)$, $25.0\left(\mathrm{CH}_{2}\right), 19.4\left(\mathrm{CH}_{3}\right), 18.5\left(\mathrm{CH}_{3}\right)$ ppm; HRMS (EI, m/z): Calcd. for $\mathrm{C}_{18} \mathrm{H}_{26} \mathrm{BrNO}_{2}$ : 367.1147, found $[\mathrm{M}-\mathrm{H}]^{+}: 366.1060$.

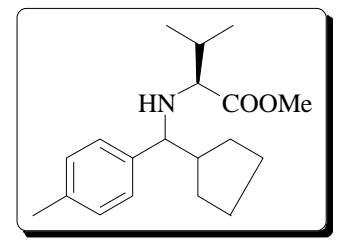

2-\{[1-Cyclopentyl-1-(p-methylphenyl)-methyl]-amino\}-3-methyl-butyric acid methyl ester (Table 4, entry 14): Colorless oil; Yield: 83\%; $\mathrm{R}_{f}=0.59$ (Ethyl acetate/Hexane 1:8); FTIR ( $\mathrm{NaCl}$, neat): $v 3447(\mathrm{NH}), 1734(\mathrm{C}=\mathrm{O}) \mathrm{cm}^{-1}$; ${ }^{1} \mathrm{H} \mathrm{NMR}\left(300 \mathrm{MHz}, \mathrm{CDCl}_{3}\right)$ : (major isomer) $\delta 0.82$ (d, $J=$ $6.75 \mathrm{~Hz}, 3 \mathrm{H}), 0.88$ (d, $J=6.78 \mathrm{~Hz}, 3 \mathrm{H}), 1.01-1.12(\mathrm{~m}, 1 \mathrm{H}), 1.17-1.27$ (m, $1 \mathrm{H}), 1.30-1.45$ (m, $2 \mathrm{H})$, 1.46-1.69 (m, 3H), 1.72-1.83 (m, 1H), 1.85-2.08 (m, 3H), 2.32 (s, 3H), 2.71 (d, $J=6.36 \mathrm{~Hz}, 1 \mathrm{H})$, 3.10 (d, $J=8.73 \mathrm{~Hz}, 1 \mathrm{H}), 3.70$ (s, 3H), 7.07-7.17 (m, 4H) ppm; ${ }^{13} \mathrm{C}$ NMR (75.4 MHz, $\mathrm{CDCl}_{3}$ ): (major isomer) $\delta 176.4$ (CO), 140.5 (C), 136.3 (C), 128.6 (CHx2), 128.0 (CHx2), 67.3 (CH), 64.3 (CH), $51.1\left(\mathrm{CH}_{3}\right), 47.6(\mathrm{CH}), 31.7(\mathrm{CH}), 30.2\left(\mathrm{CH}_{2}\right), 30.0\left(\mathrm{CH}_{2}\right), 25.4\left(\mathrm{CH}_{2}\right), 25.0\left(\mathrm{CH}_{2}\right), 21.1$ $\left(\mathrm{CH}_{3}\right), 19.4\left(\mathrm{CH}_{3}\right), 18.6\left(\mathrm{CH}_{3}\right)$ ppm; HRMS (EI, m/z): Calcd. for $\mathrm{C}_{19} \mathrm{H}_{29} \mathrm{NO}_{2}$ : 303.2198, found $[\mathrm{M}-\mathrm{H}]^{+}: 302.2123$.

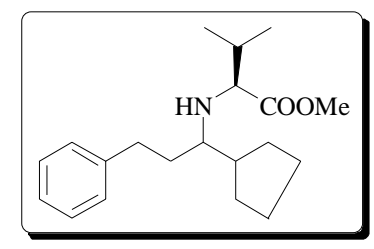

2-[(1-Cyclopentyl-3-phenyl-propyl)-amino]-3-methyl-butyric acid methyl ester (Table 4, 
entry 15): Colorless oil; Yield: 71\%; $\mathrm{R}_{f}=0.43$ and 0.55 (Ethyl acetate/Hexane 1:8); FTIR ( $\mathrm{NaCl}$, neat): $v 3449(\mathrm{NH}), 1736(\mathrm{C}=\mathrm{O}) \mathrm{cm}^{-1}$; ${ }^{1} \mathrm{H} \mathrm{NMR}\left(300 \mathrm{MHz}, \mathrm{CDCl}_{3}\right.$ ): (major isomer) $\delta 0.96$ (d, $J=$ $6.72 \mathrm{~Hz}, 3 \mathrm{H}), 1.00$ (d, $J=6.75 \mathrm{~Hz}, 3 \mathrm{H}), 1.20-1.26(\mathrm{~m}, 2 \mathrm{H}), 1.50-2.00$ (m, 11H), 2.24-2.30 (m, $1 \mathrm{H})$, 2.52-2.82 (m, 2H), 3.07 (d, $J=6.63 \mathrm{~Hz}, 1 \mathrm{H}), 3.68$ (s, 3H), 7.15-7.29 (m, 5H) ppm; ${ }^{13} \mathrm{C}$ NMR (75.4 MHz, $\mathrm{CDCl}_{3}$ ): (major isomer) $\delta 176.3$ (CO), 143.4 (C), 128.3 (CHx2), 128.3 (CHx2), 125.5 $(\mathrm{CH}), 64.9(\mathrm{CH}), 60.7(\mathrm{CH}), 51.2\left(\mathrm{CH}_{3}\right), 43.0(\mathrm{CH}), 33.5\left(\mathrm{CH}_{2}\right), 32.0(\mathrm{CH}), 30.0\left(\mathrm{CH}_{2}\right), 29.7$ $\left(\mathrm{CH}_{2}\right)$, $29.6\left(\mathrm{CH}_{2}\right), 25.5\left(\mathrm{CH}_{2}\right), 25.4\left(\mathrm{CH}_{2}\right), 19.6\left(\mathrm{CH}_{3}\right), 19.0\left(\mathrm{CH}_{3}\right)$ ppm; HRMS (EI, m/z): Calcd. for $\mathrm{C}_{20} \mathrm{H}_{31} \mathrm{NO}_{2}$ : 317.2355, found [M-H] : 316.2285 .

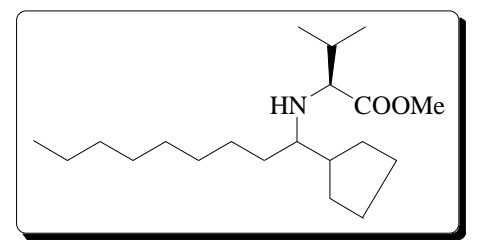

2-[(1-Cyclopentyl-nonyl)-amino]-3-methyl-butyric acid methyl ester (Table 4, entry 16): Colorless oil; Yield: 69\%; $\mathrm{R}_{f}=0.52$ and 0.61 (Ethyl acetate/Hexane 1:8); FTIR ( $\mathrm{NaCl}$, neat): $v$ $3455(\mathrm{NH}), 1736(\mathrm{C}=\mathrm{O}) \mathrm{cm}^{-1}$; ${ }^{1} \mathrm{H}$ NMR (400 MHz, $\left.\mathrm{CDCl}_{3}\right)$ : (major isomer) $\delta$ 0.86-0.96 (m, 9H), 1.26-1.90 (m, 25H), 2.17-2.19 (m, 1H), 3.03 (d, $J=6.40 \mathrm{~Hz}, 1 \mathrm{H}), 3.69$ (s, 3H) ppm; ${ }^{13} \mathrm{C}$ NMR (100 MHz, $\mathrm{CDCl}_{3}$ ): (major isomer) $\delta 176.3(\mathrm{CO}), 64.9(\mathrm{CH}), 60.9(\mathrm{CH}), 51.1\left(\mathrm{CH}_{3}\right), 43.3(\mathrm{CH})$, $32.0(\mathrm{CH}), 31.9\left(\mathrm{CH}_{2}\right), 31.5\left(\mathrm{CH}_{2}\right), 30.3\left(\mathrm{CH}_{2}\right), 29.6\left(\mathrm{CH}_{2}\right), 29.6\left(\mathrm{CH}_{2}\right), 29.4\left(\mathrm{CH}_{2}\right), 29.3\left(\mathrm{CH}_{2}\right)$, $25.5\left(\mathrm{CH}_{2}\right), 25.4\left(\mathrm{CH}_{2}\right), 23.7\left(\mathrm{CH}_{2}\right), 22.7\left(\mathrm{CH}_{2}\right), 19.4\left(\mathrm{CH}_{3}\right), 19.0\left(\mathrm{CH}_{3}\right), 14.1\left(\mathrm{CH}_{3}\right) \mathrm{ppm}$; HRMS (EI, m/z): Calcd. for $\mathrm{C}_{20} \mathrm{H}_{39} \mathrm{NO}_{2}$ : 325.2981, found [M-H] $]^{+}$: 324.2899. 


\section{$\underline{{ }^{1} \mathrm{H} \text { and }{ }^{13} \mathrm{C} \text { NMR Spectra of Products }}$}
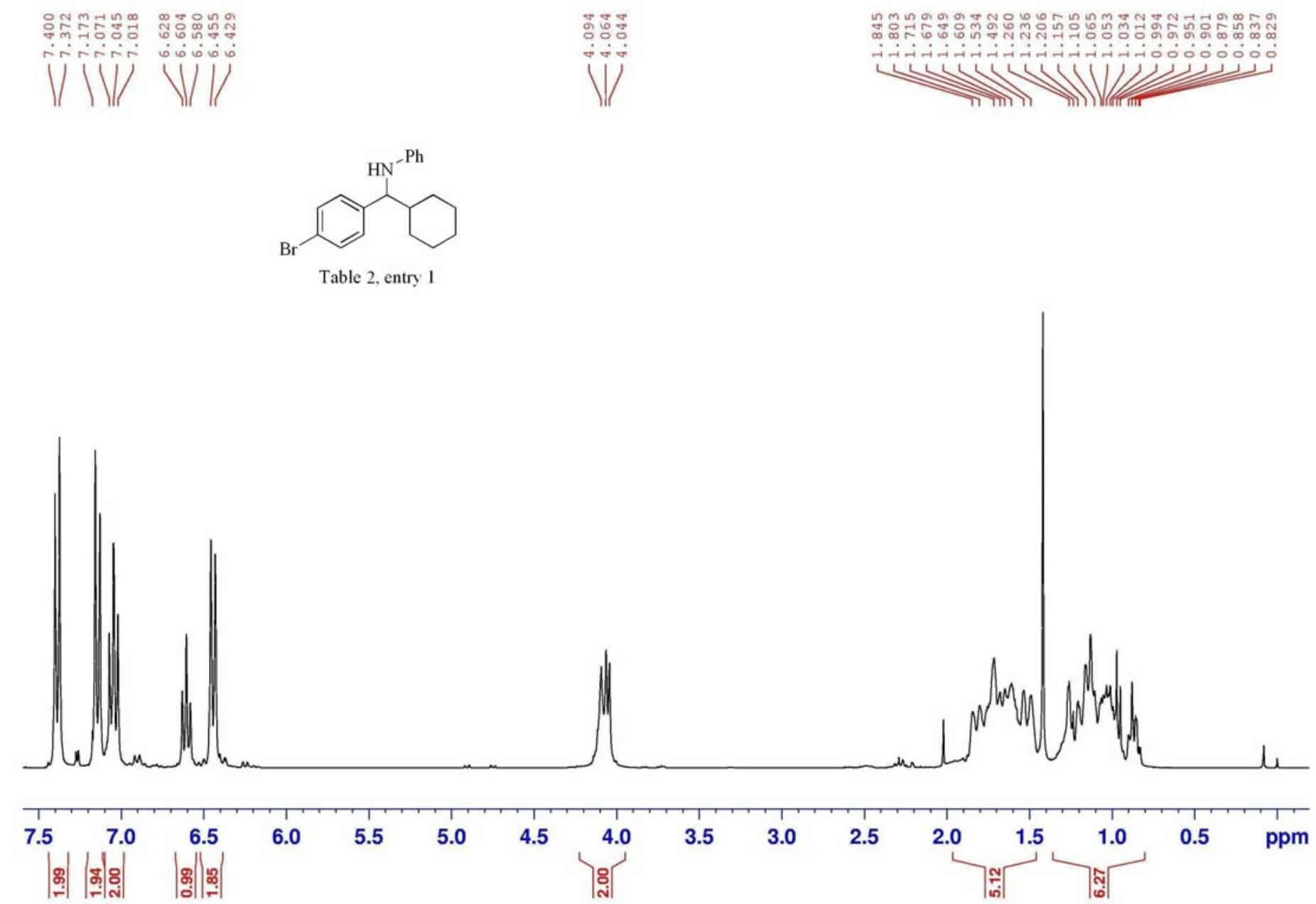


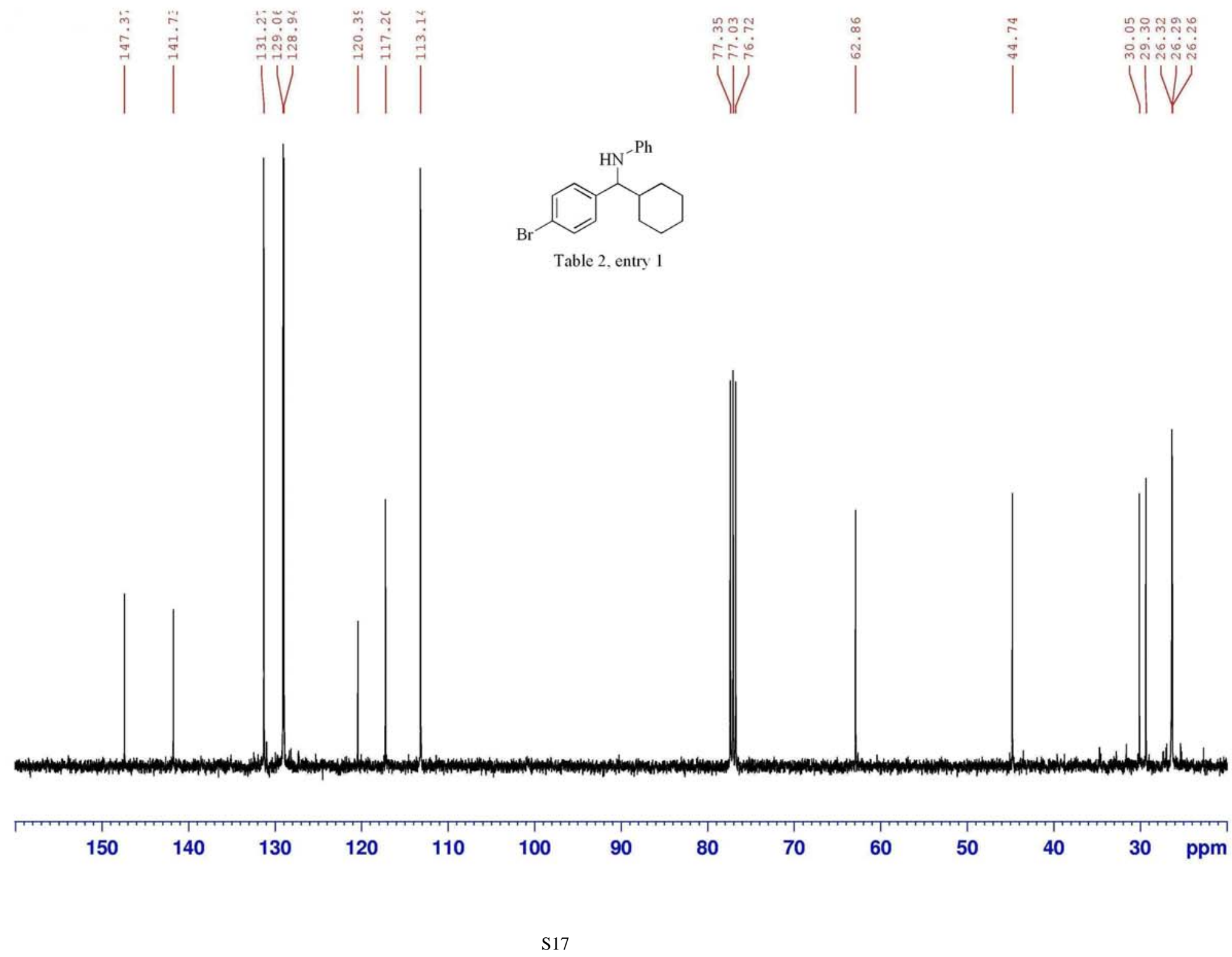


ñ

Nill

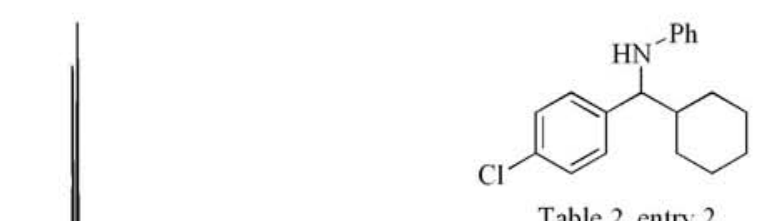

Table 2. entry 2

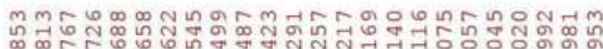
(1) $\ldots 1011111$ 


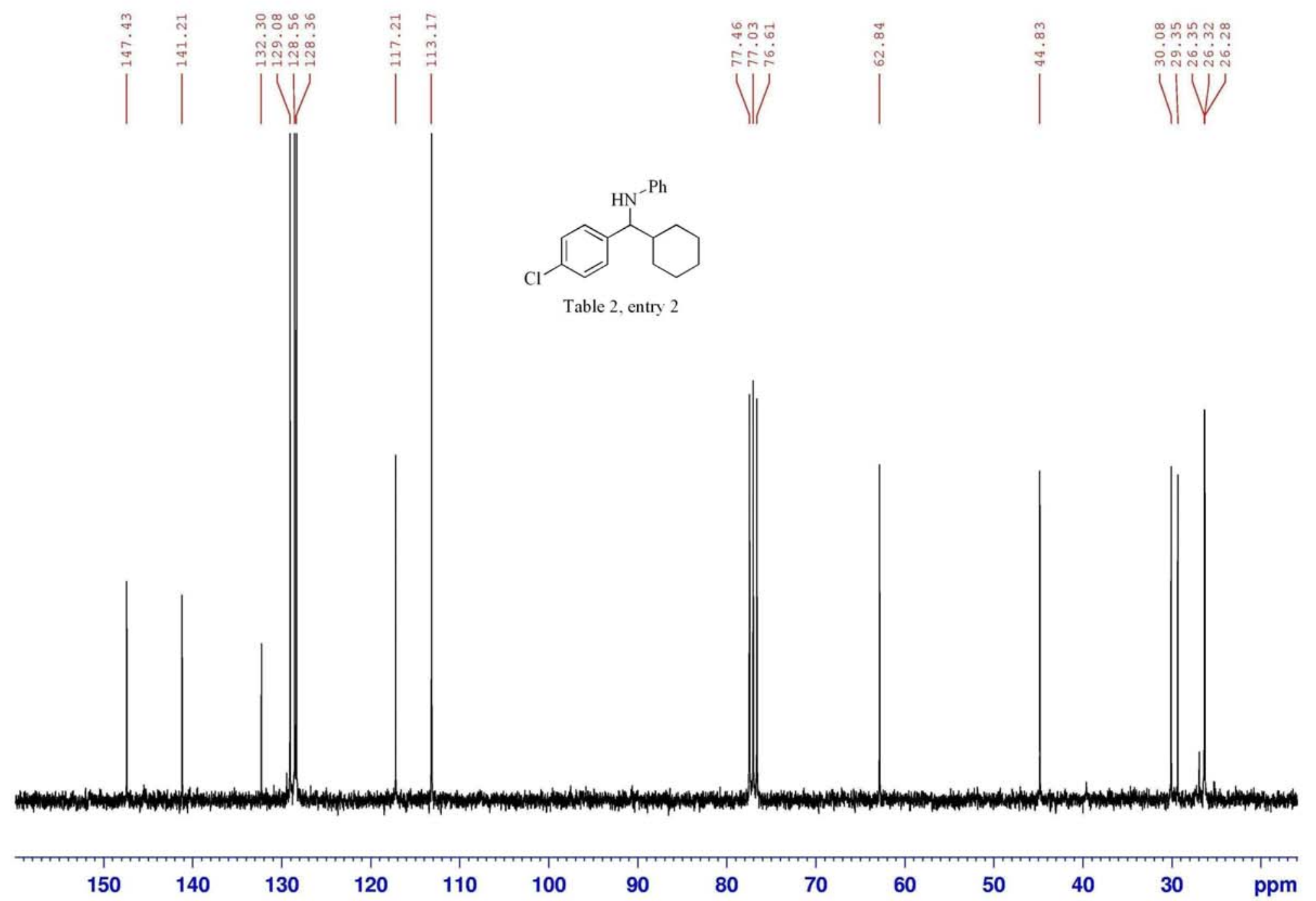




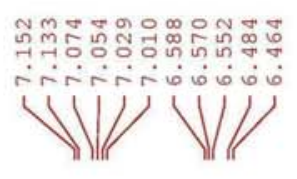<smiles>Cc1ccc(C(Nc2ccccc2)C2CCCCC2)cc1</smiles>

Table 2, entry 3
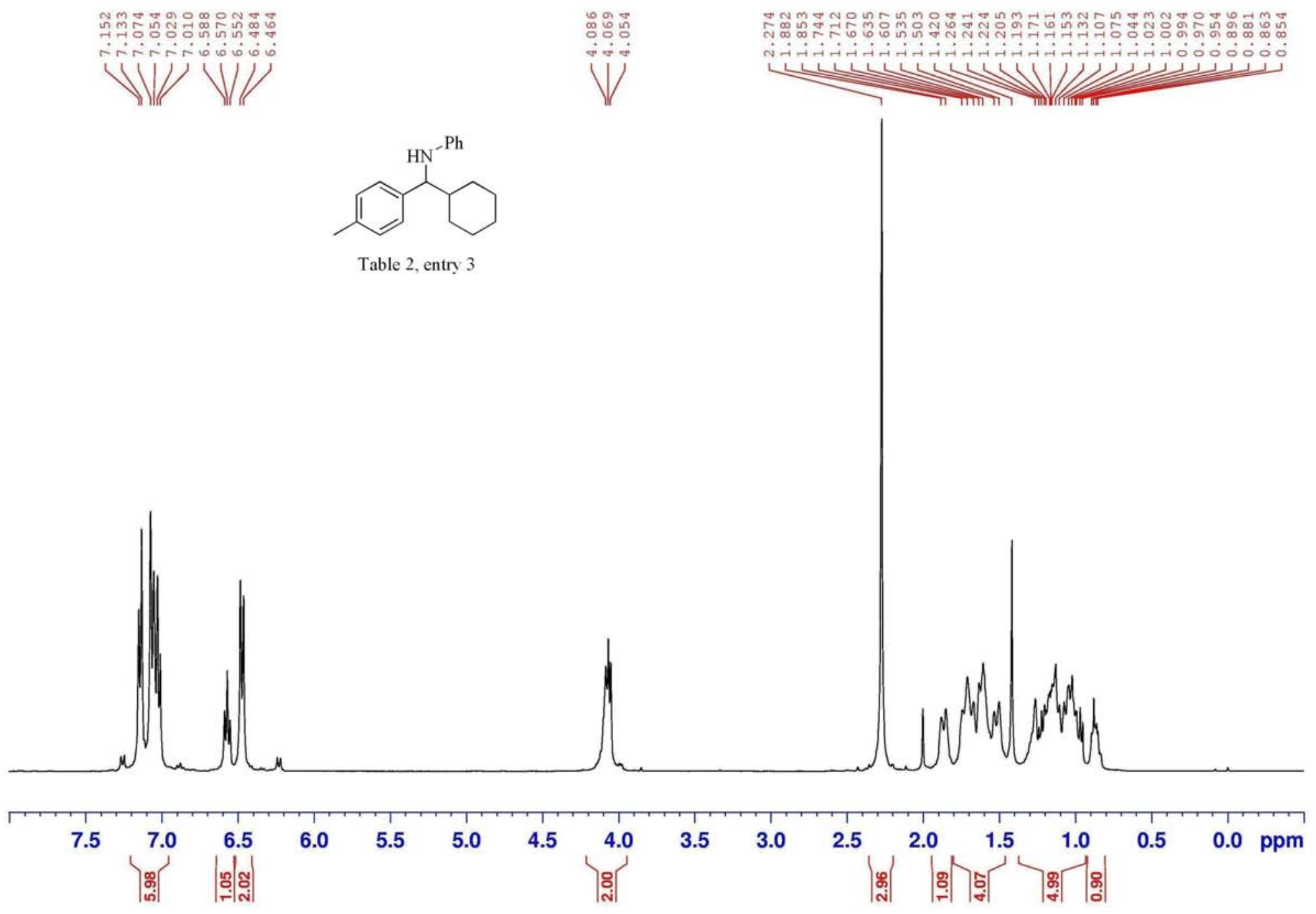

S20 


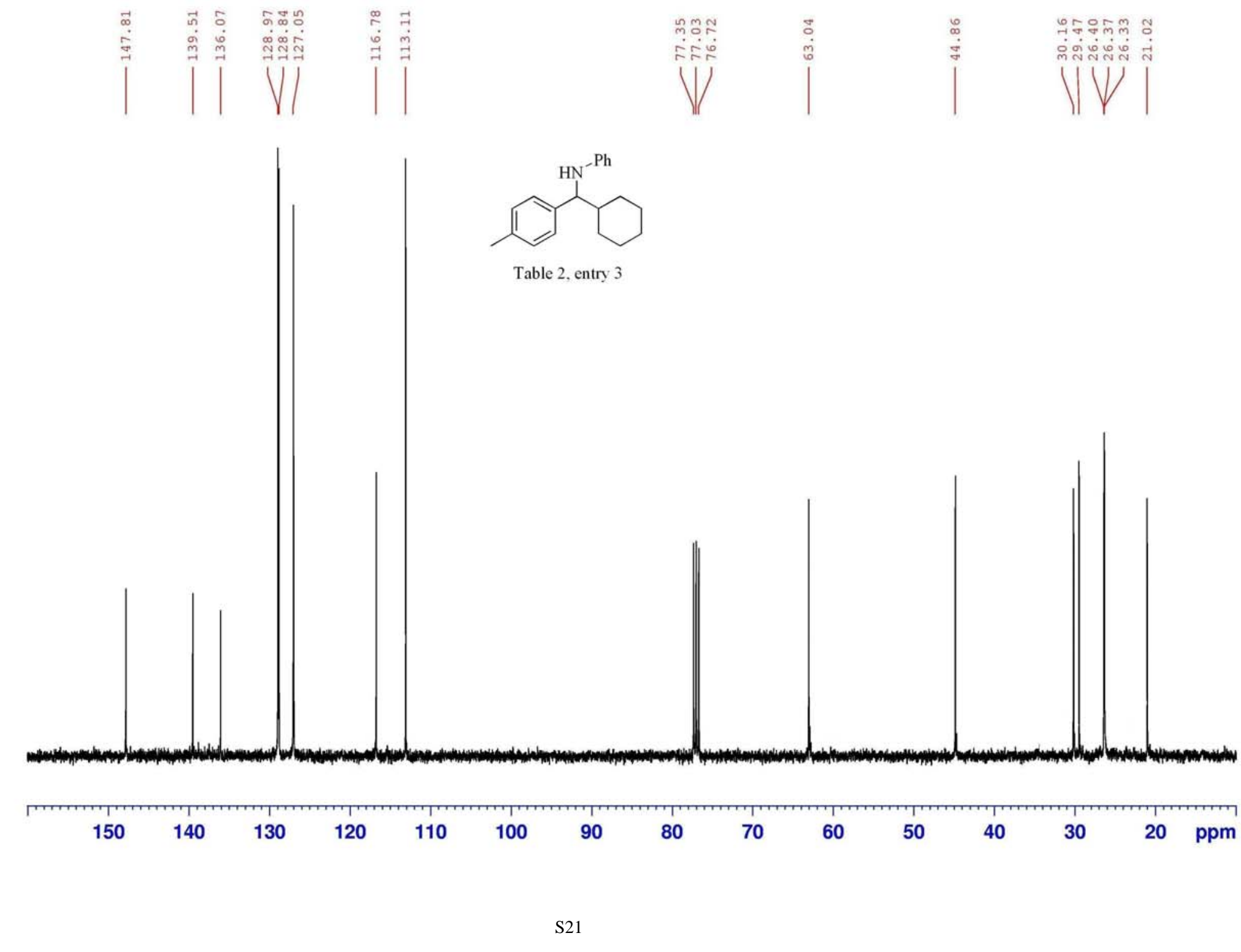




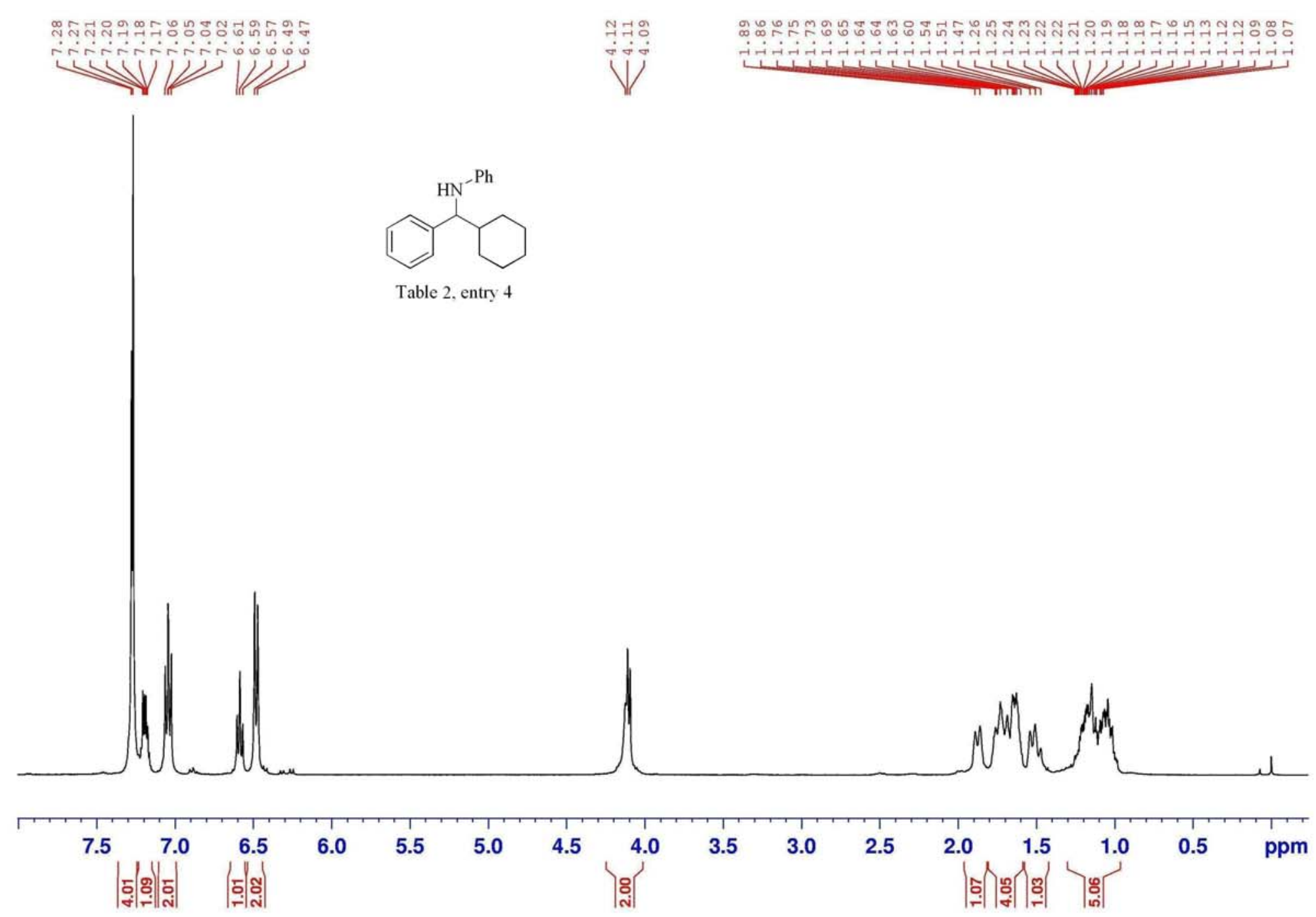



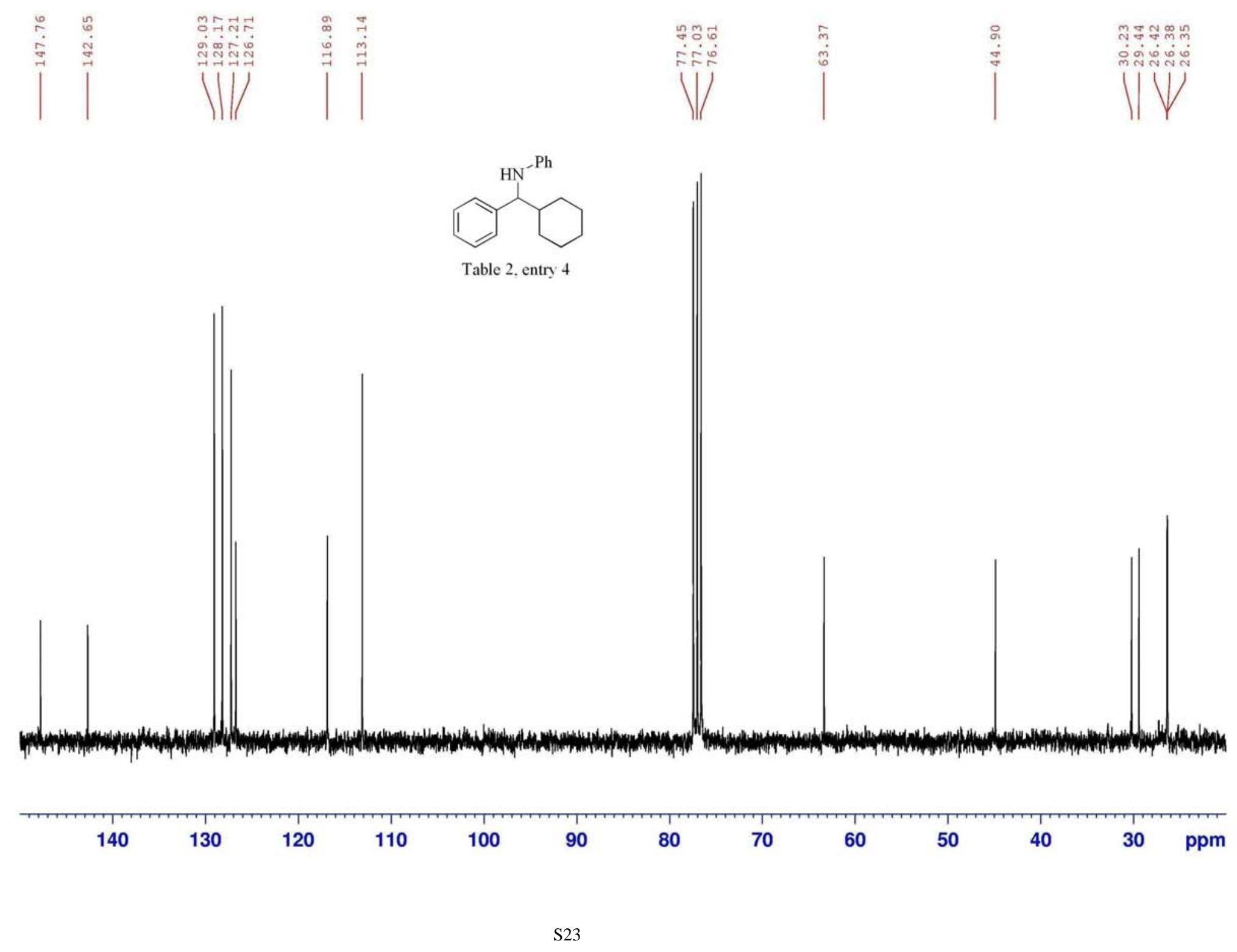


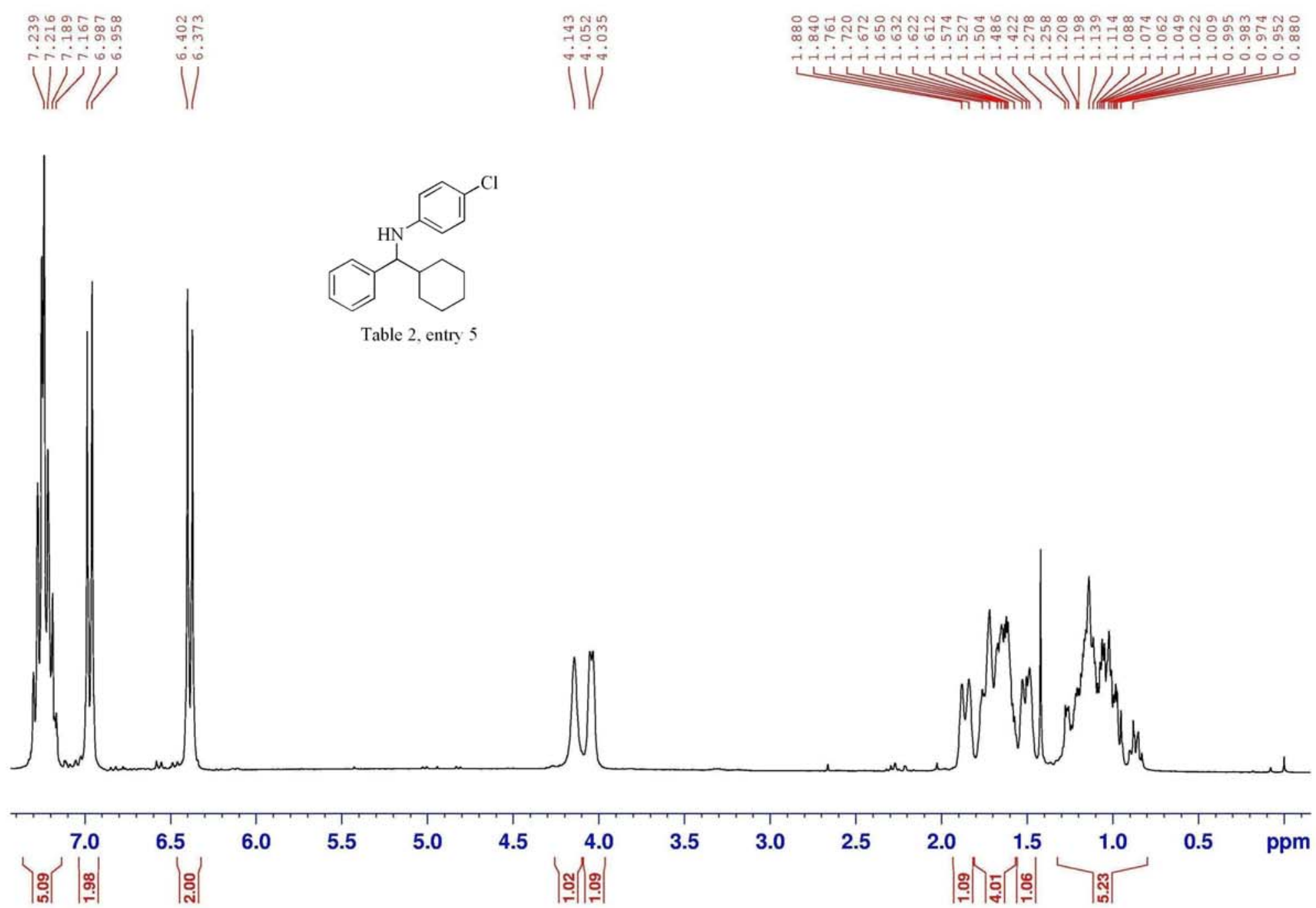



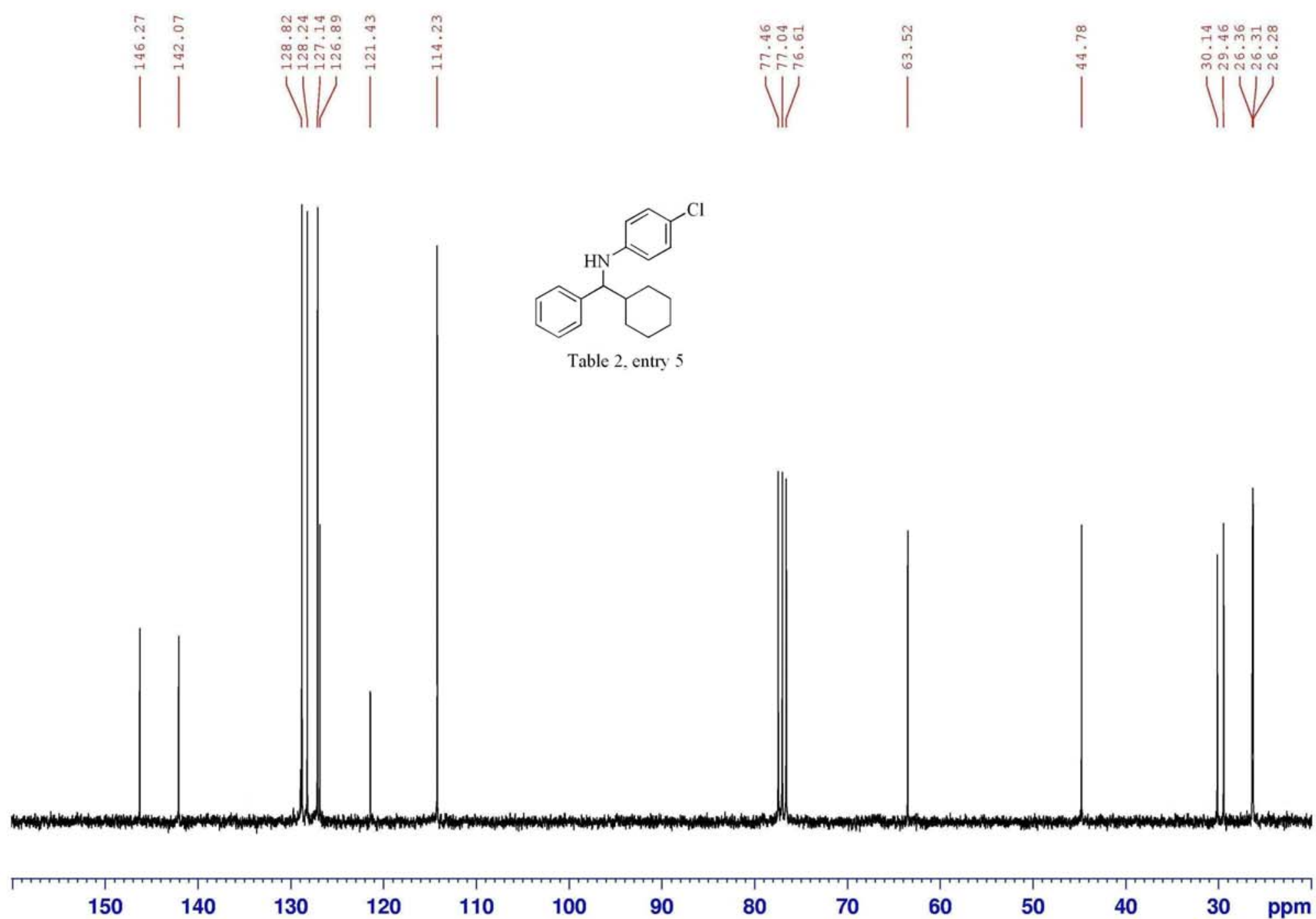


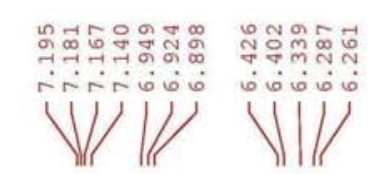

Vு

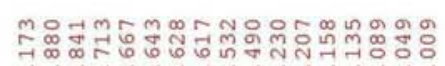

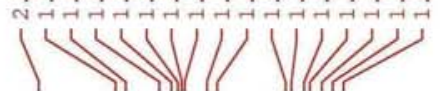

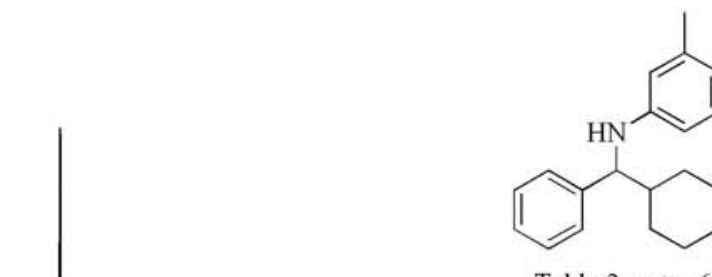

Table 2, entry 6

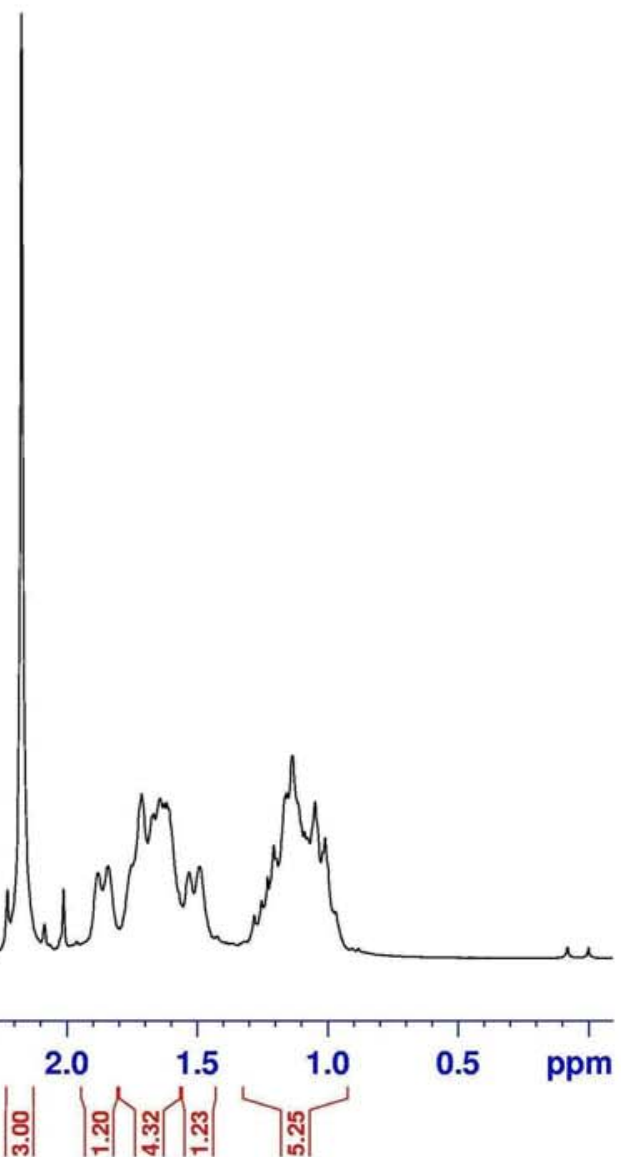



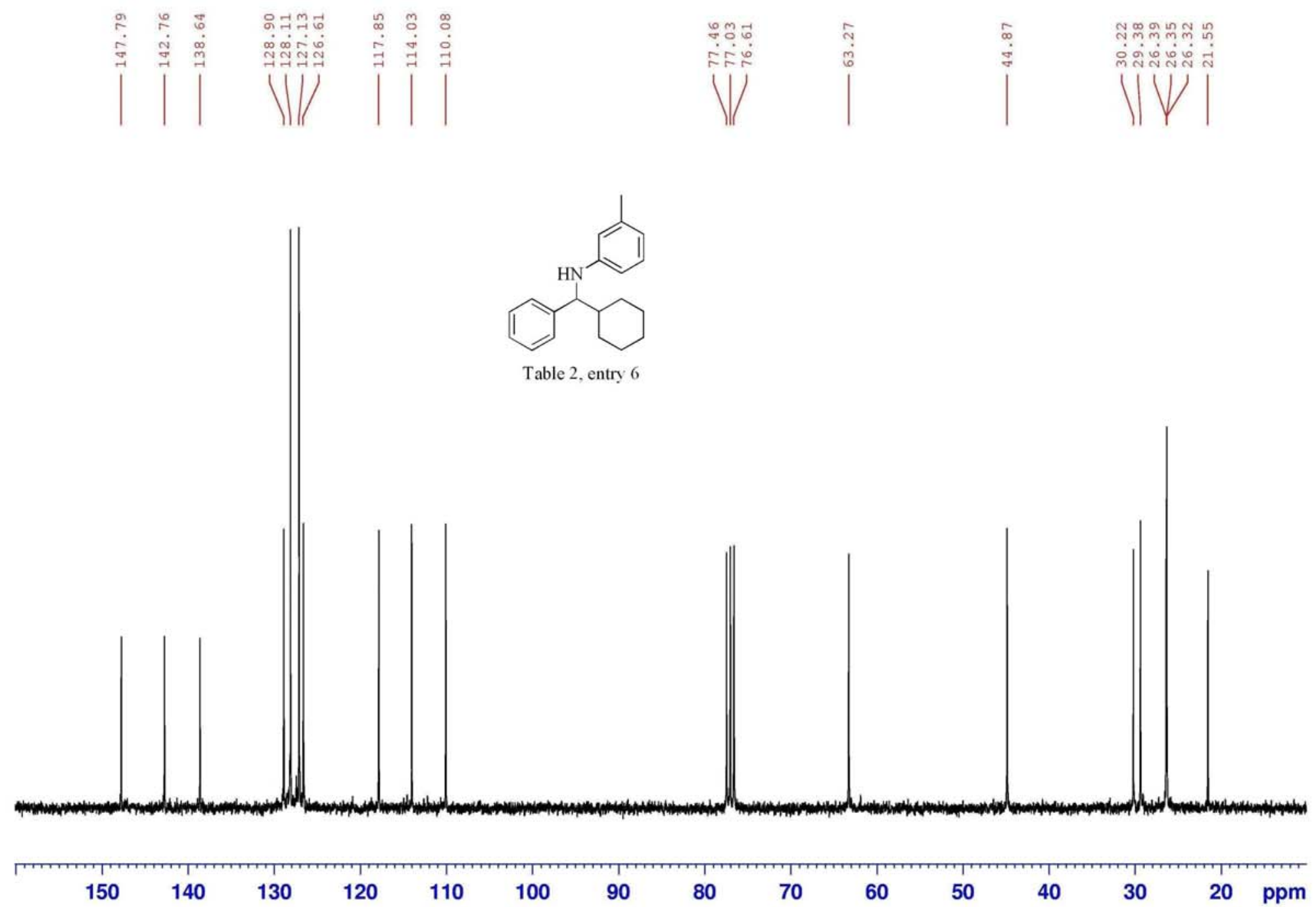


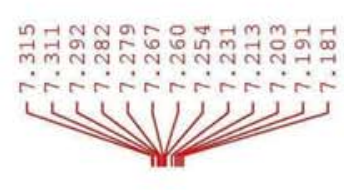

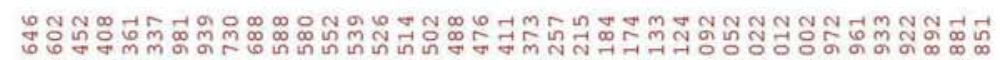

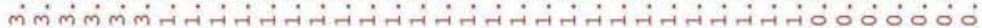

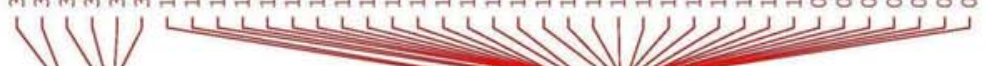

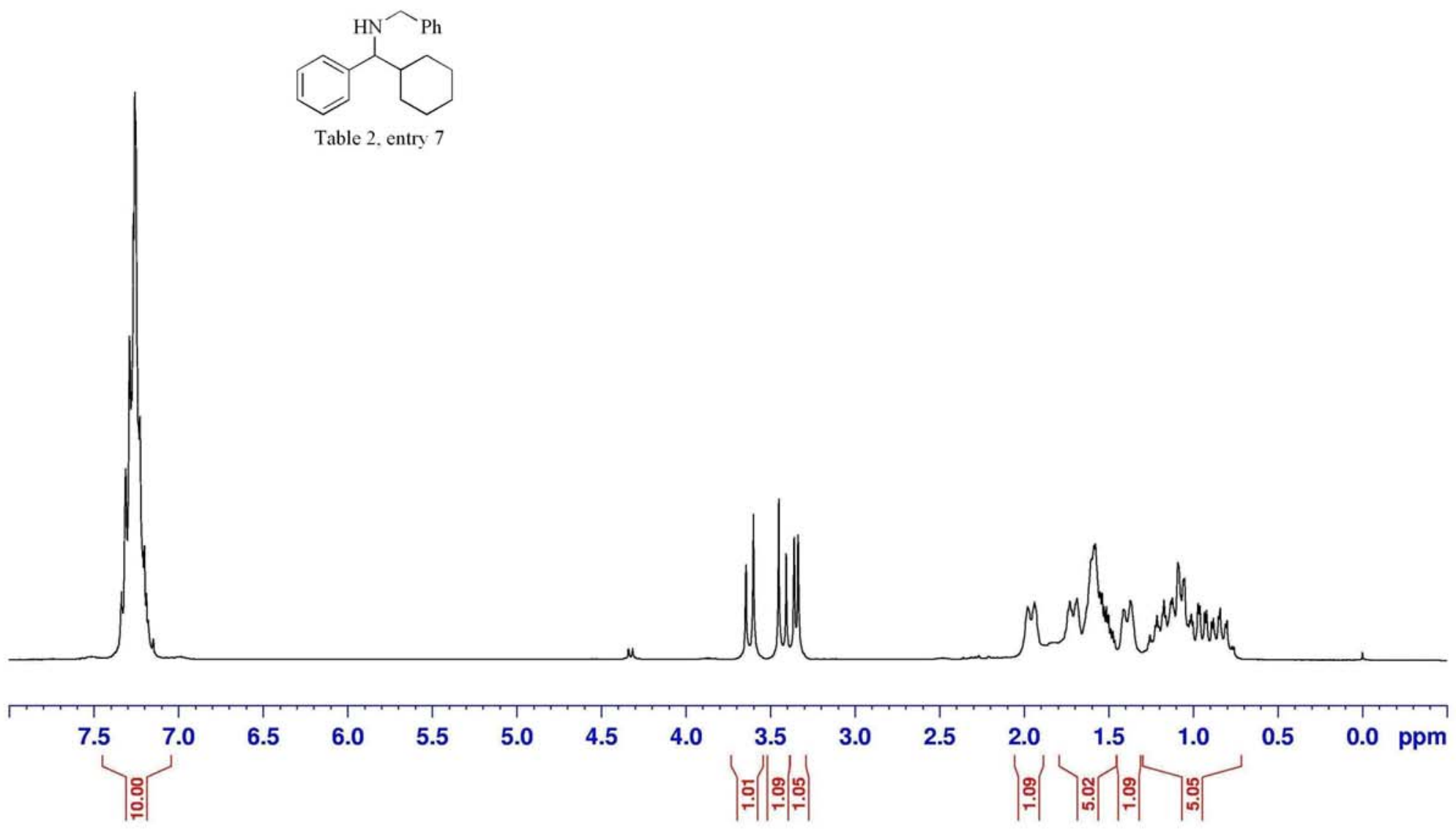




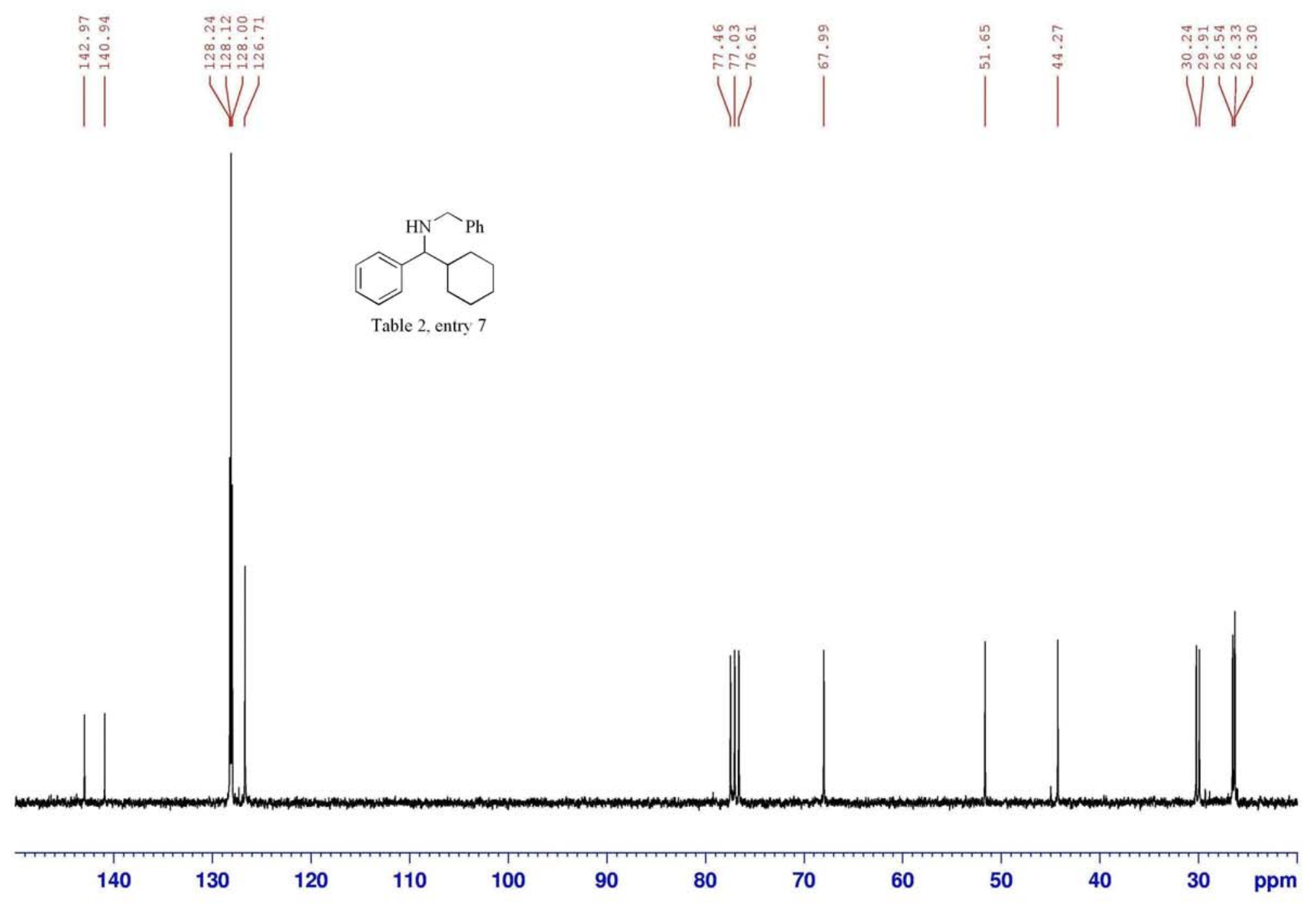




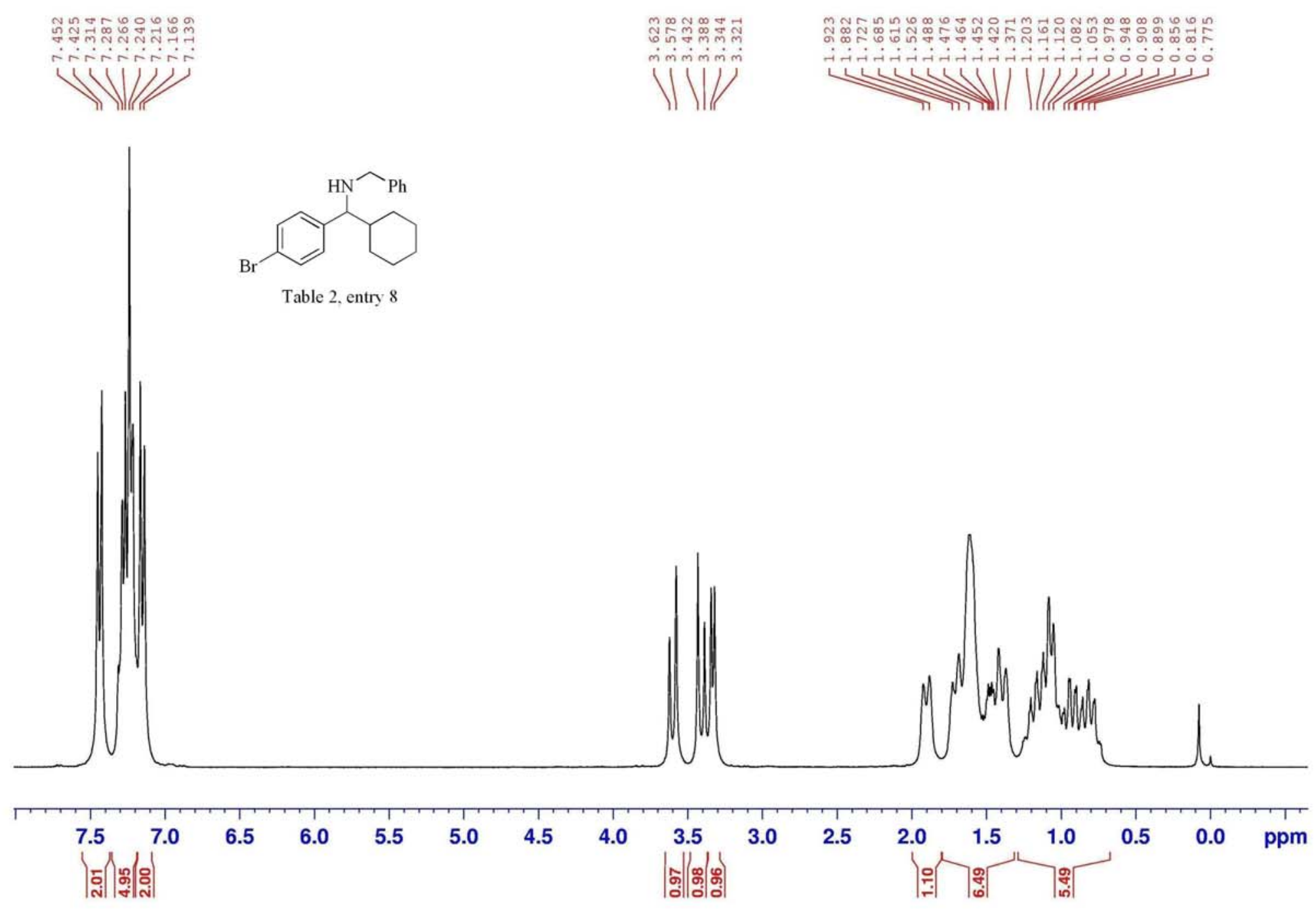



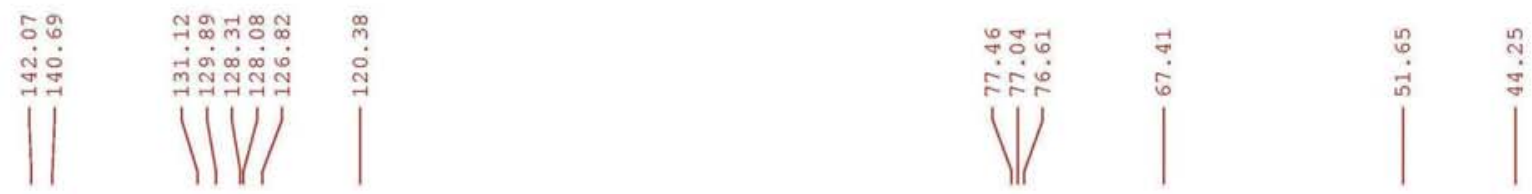

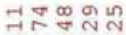

กักสักับ

UV

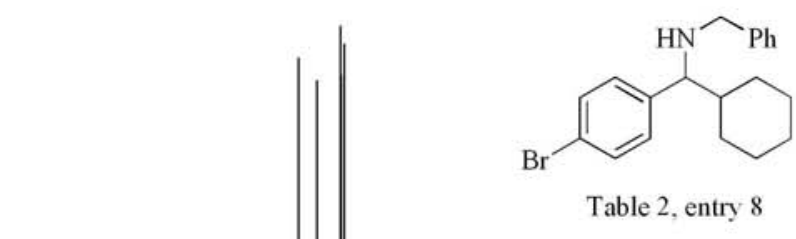




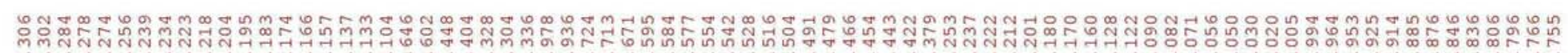

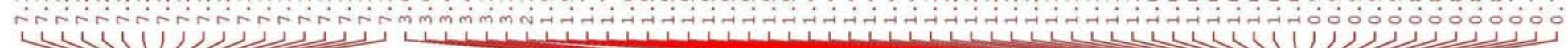

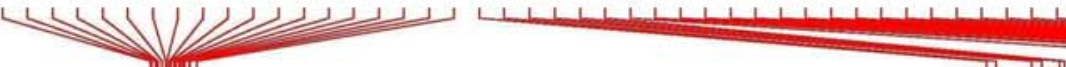

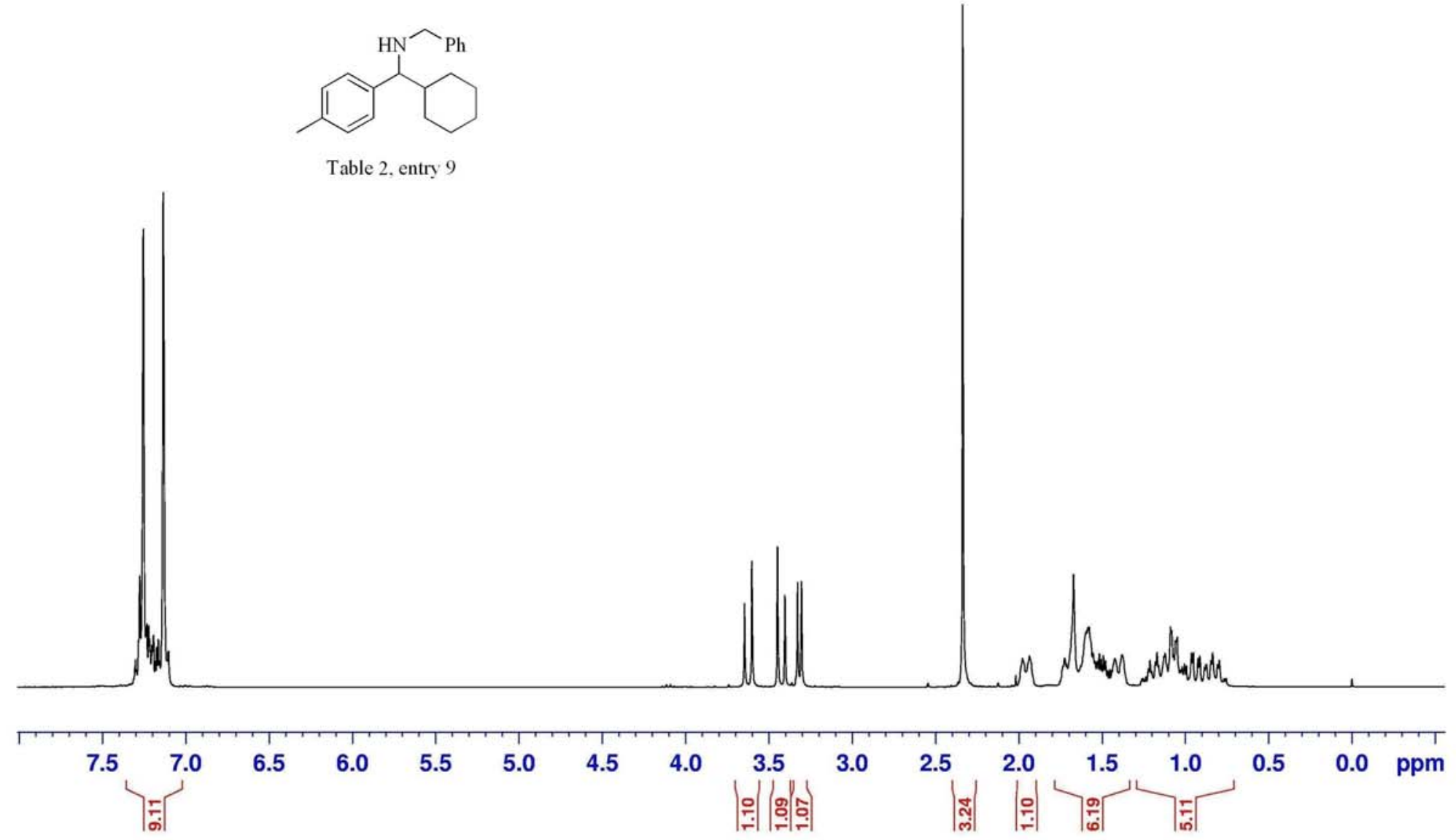




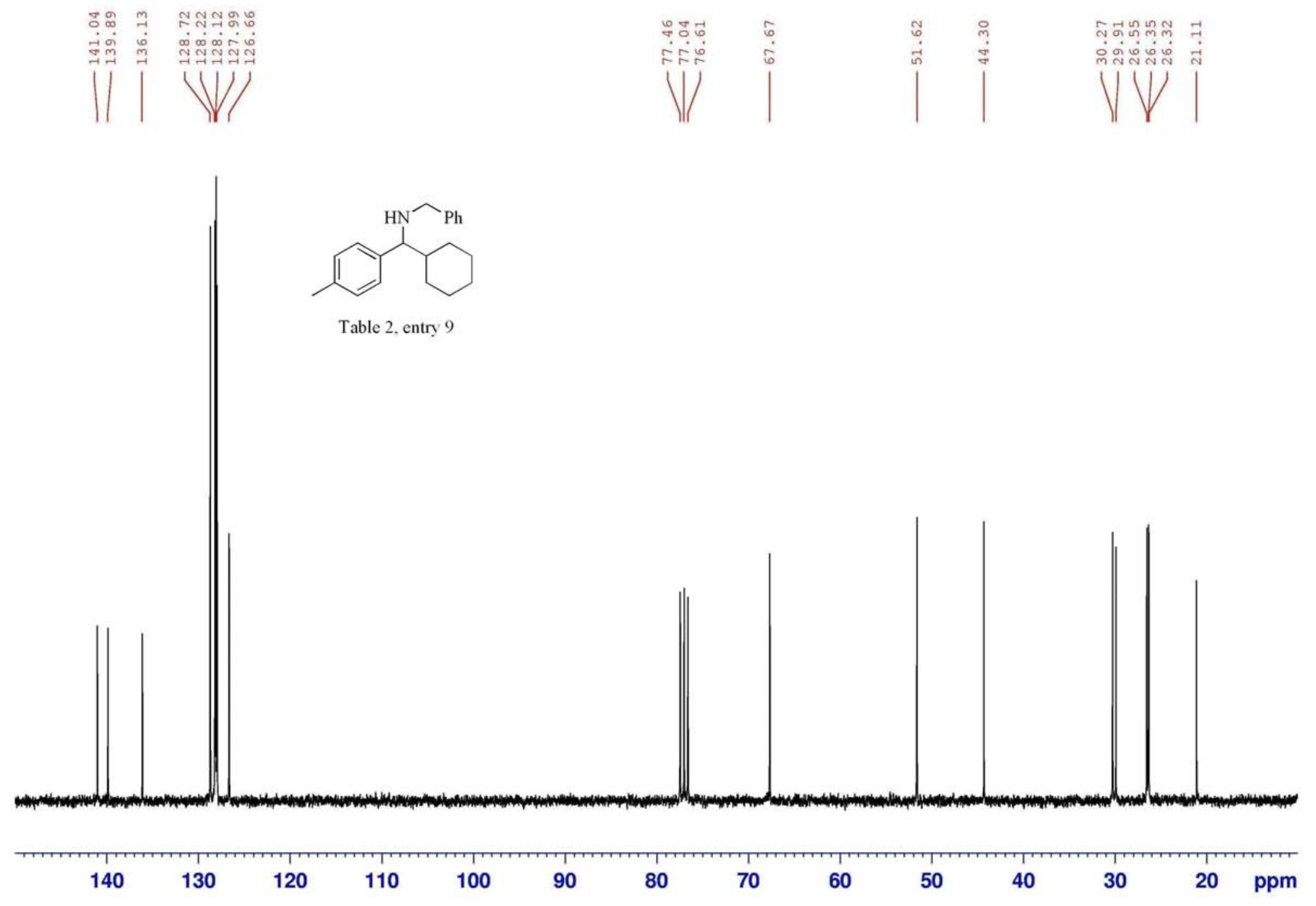



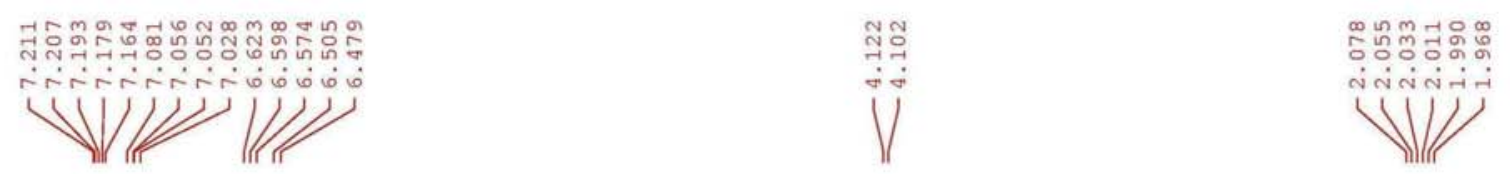

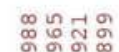

iij

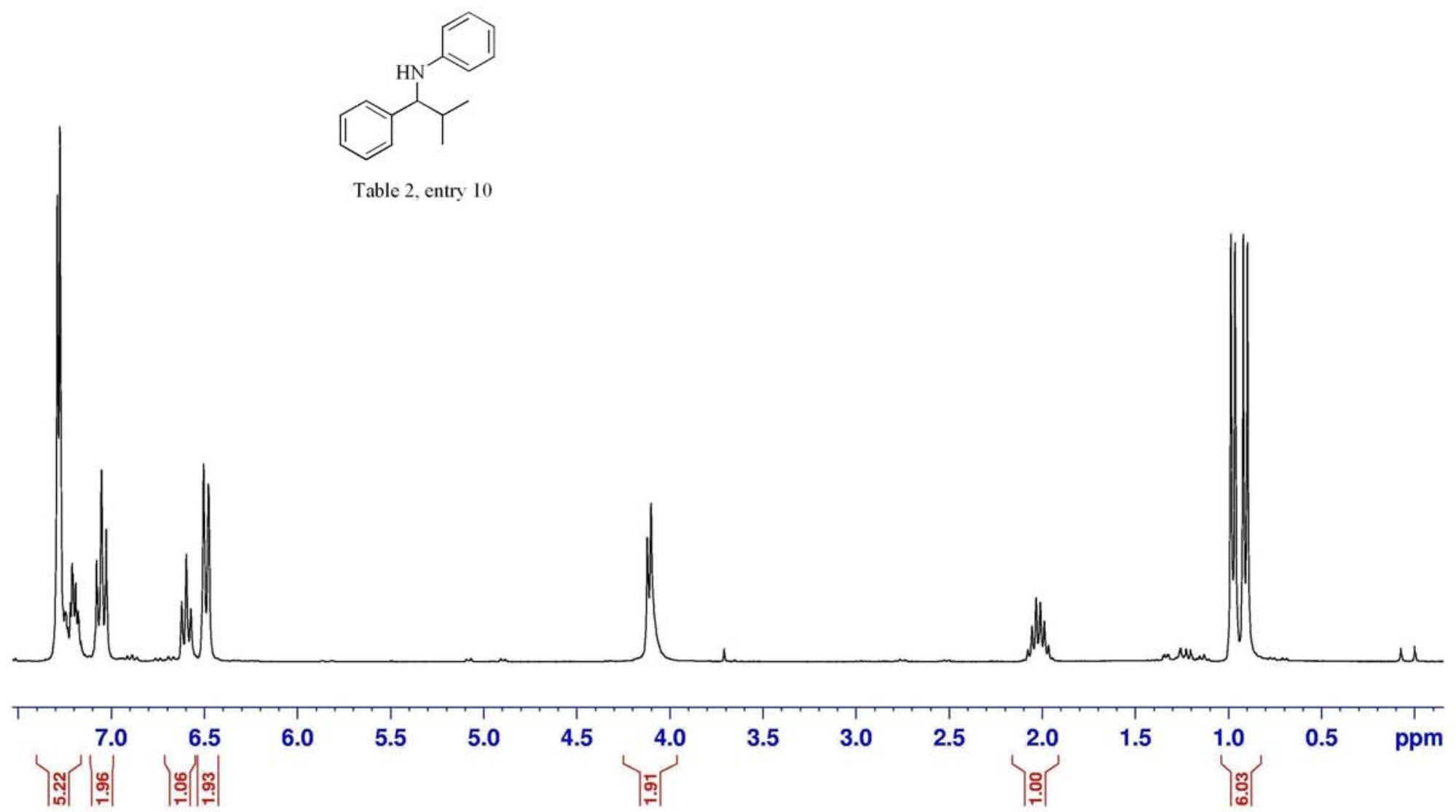




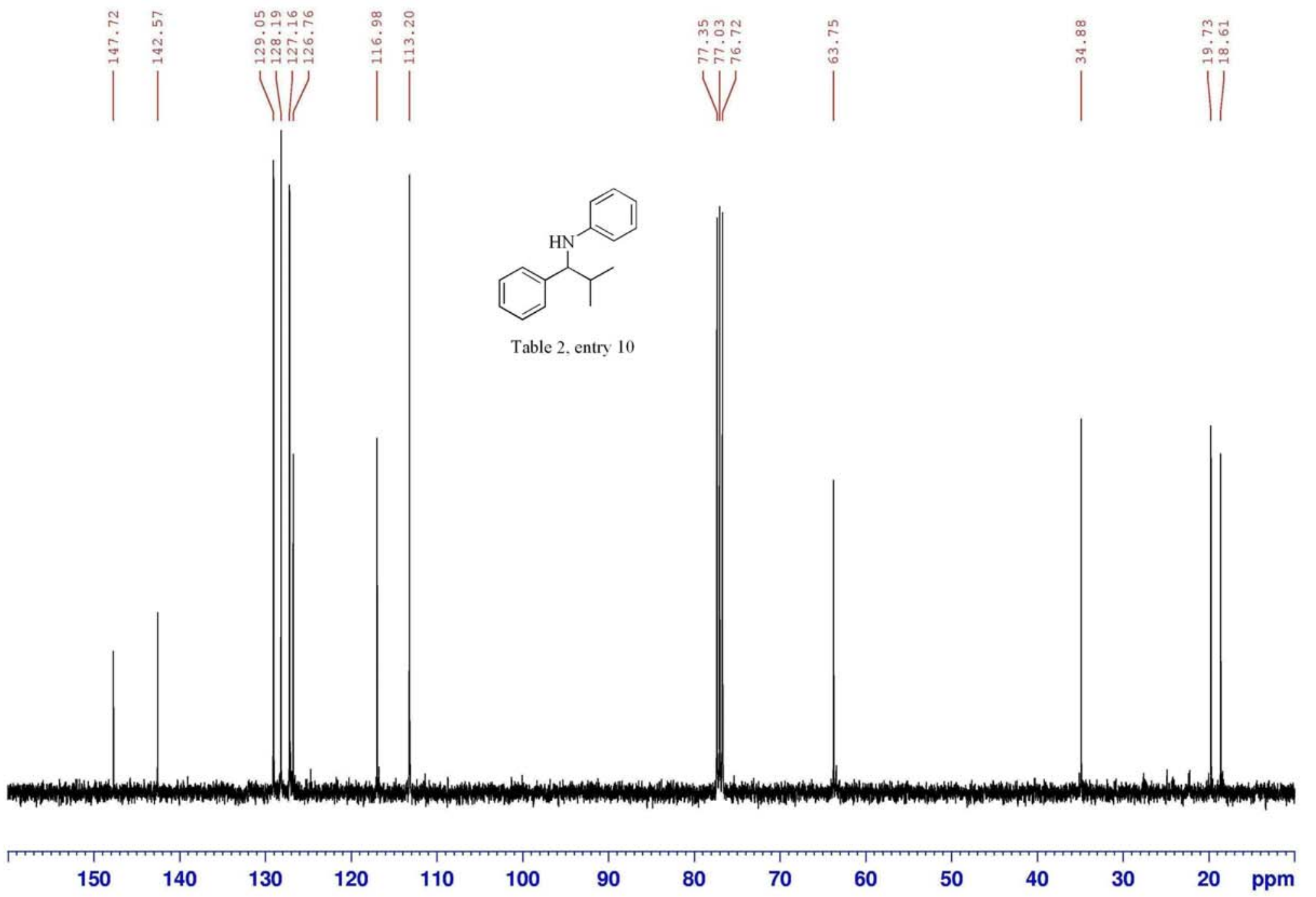




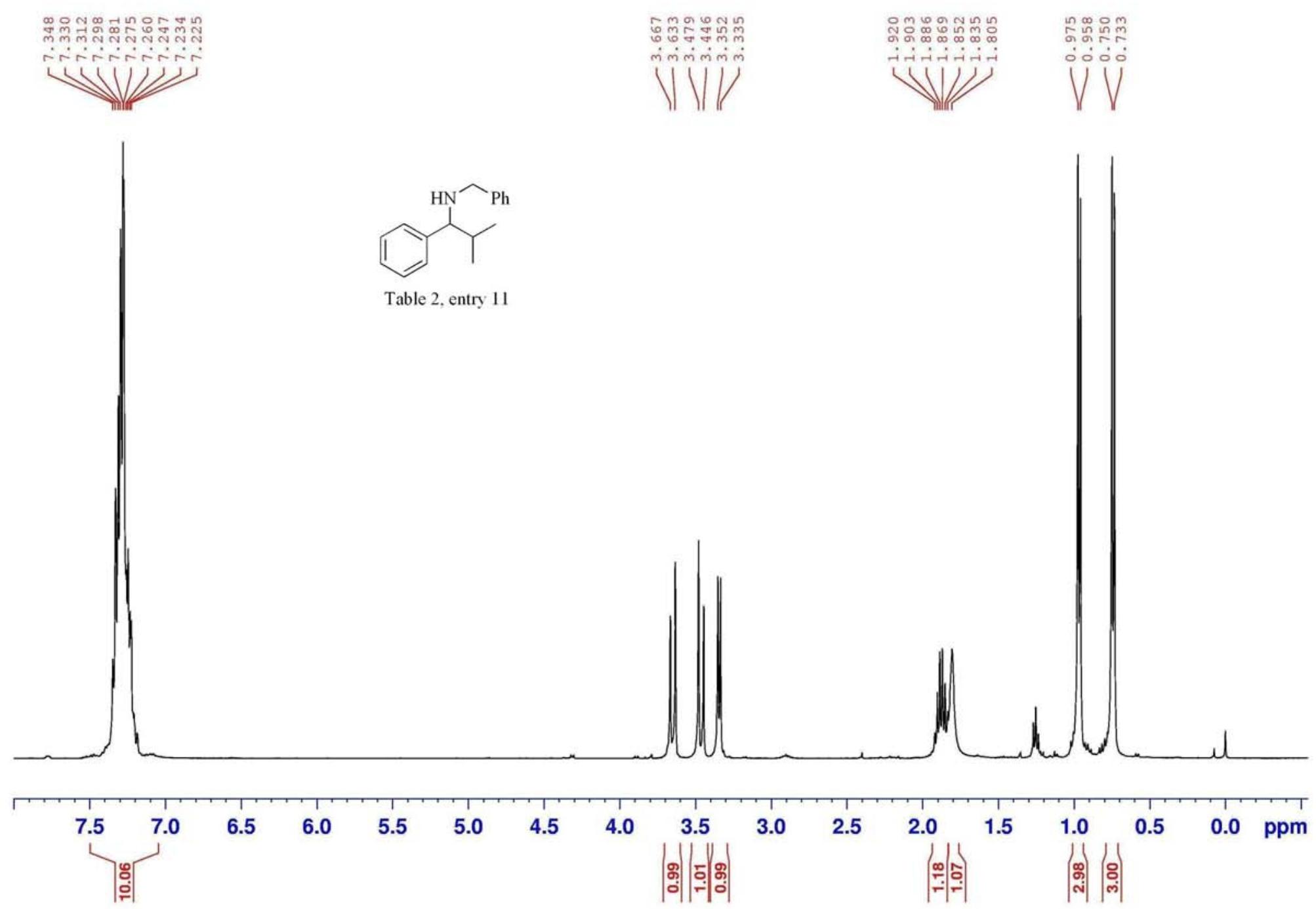




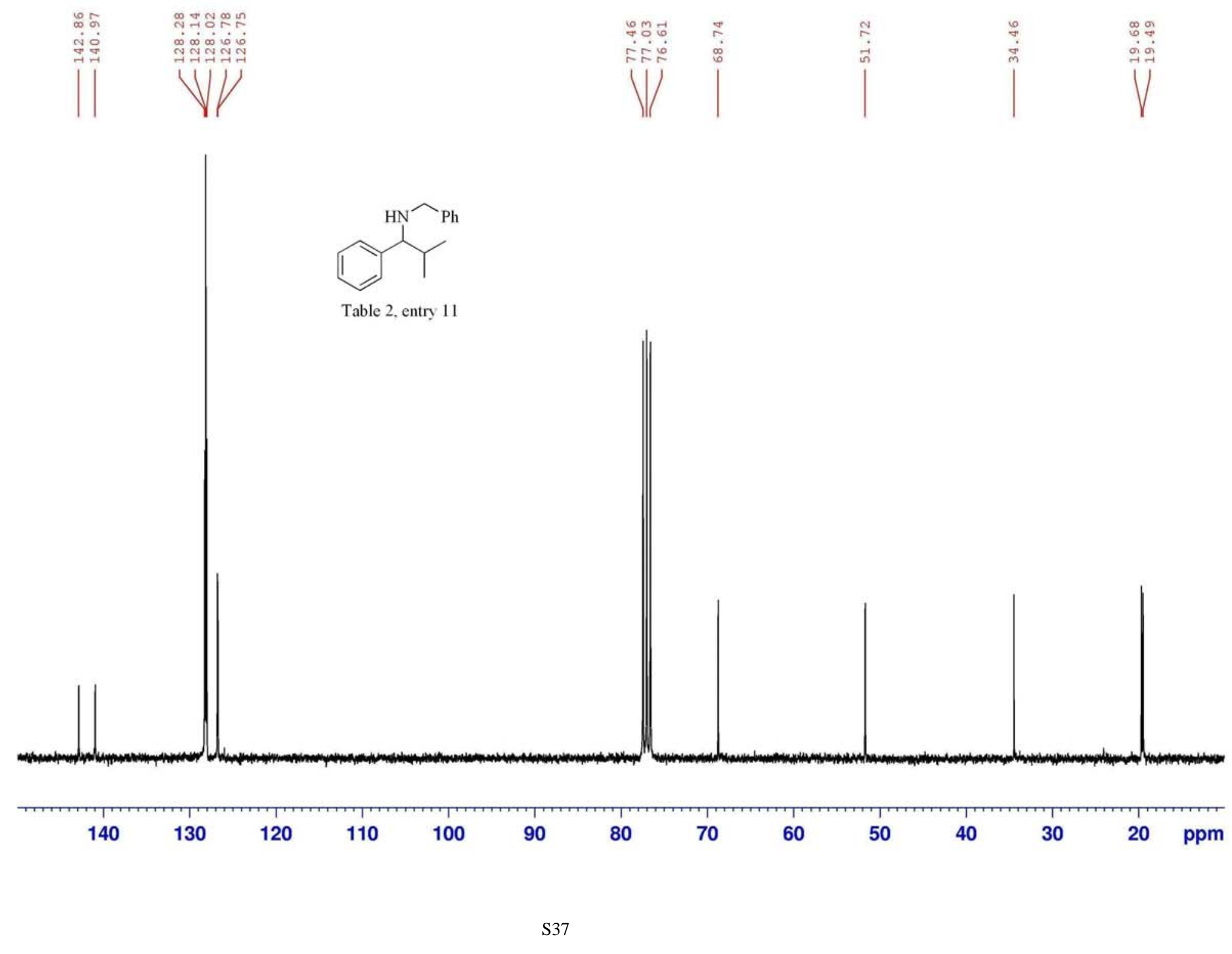




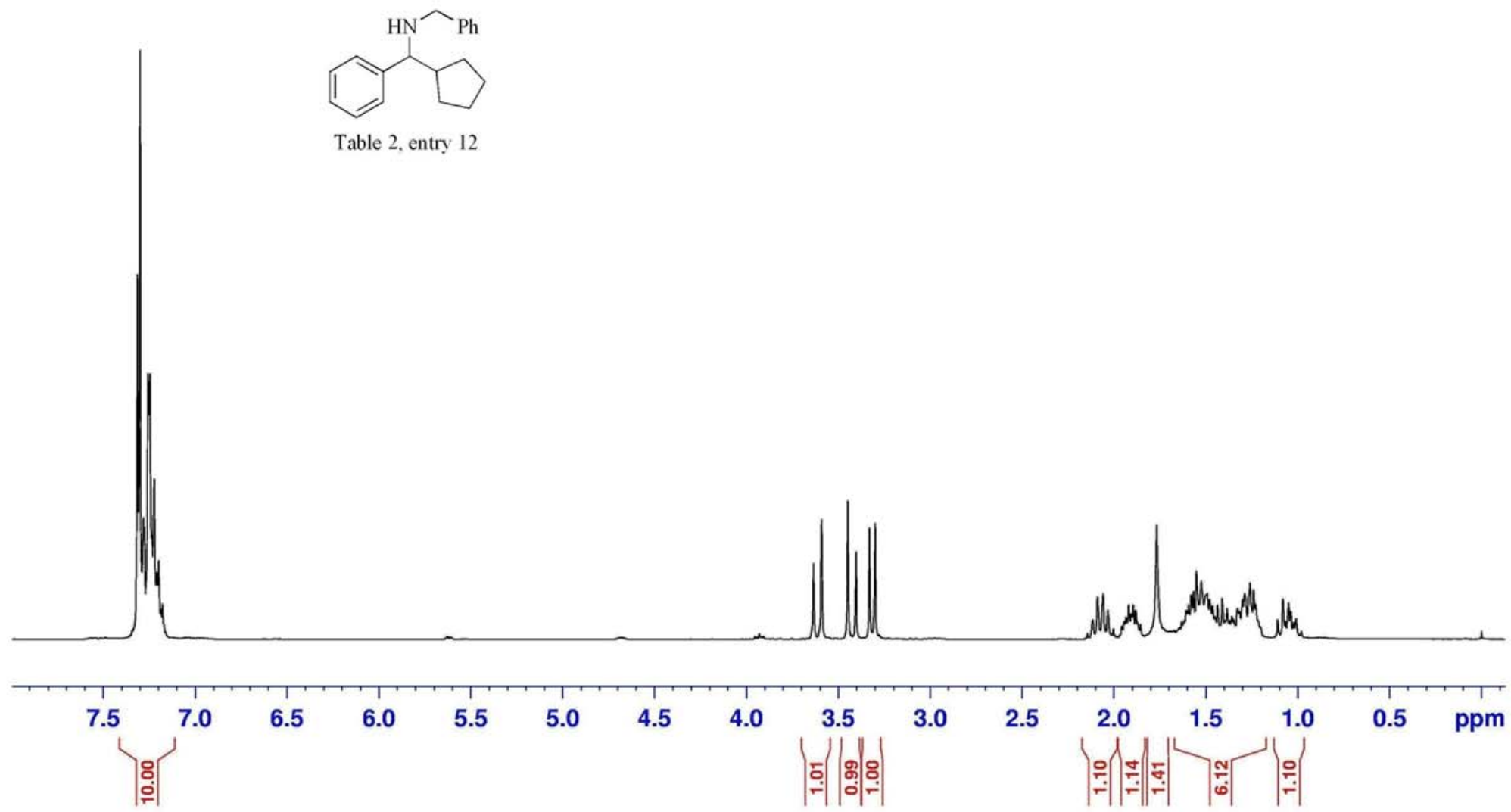




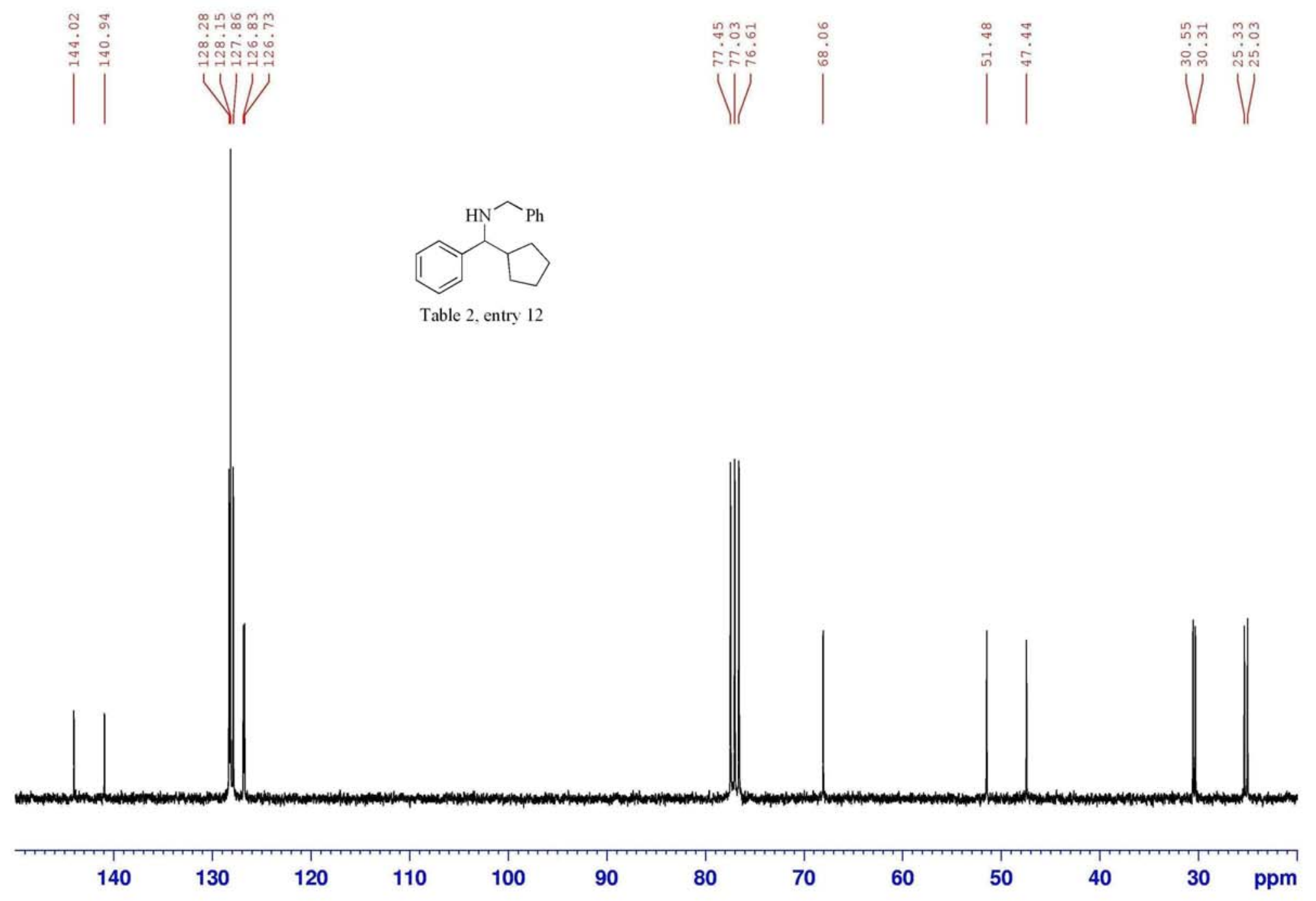



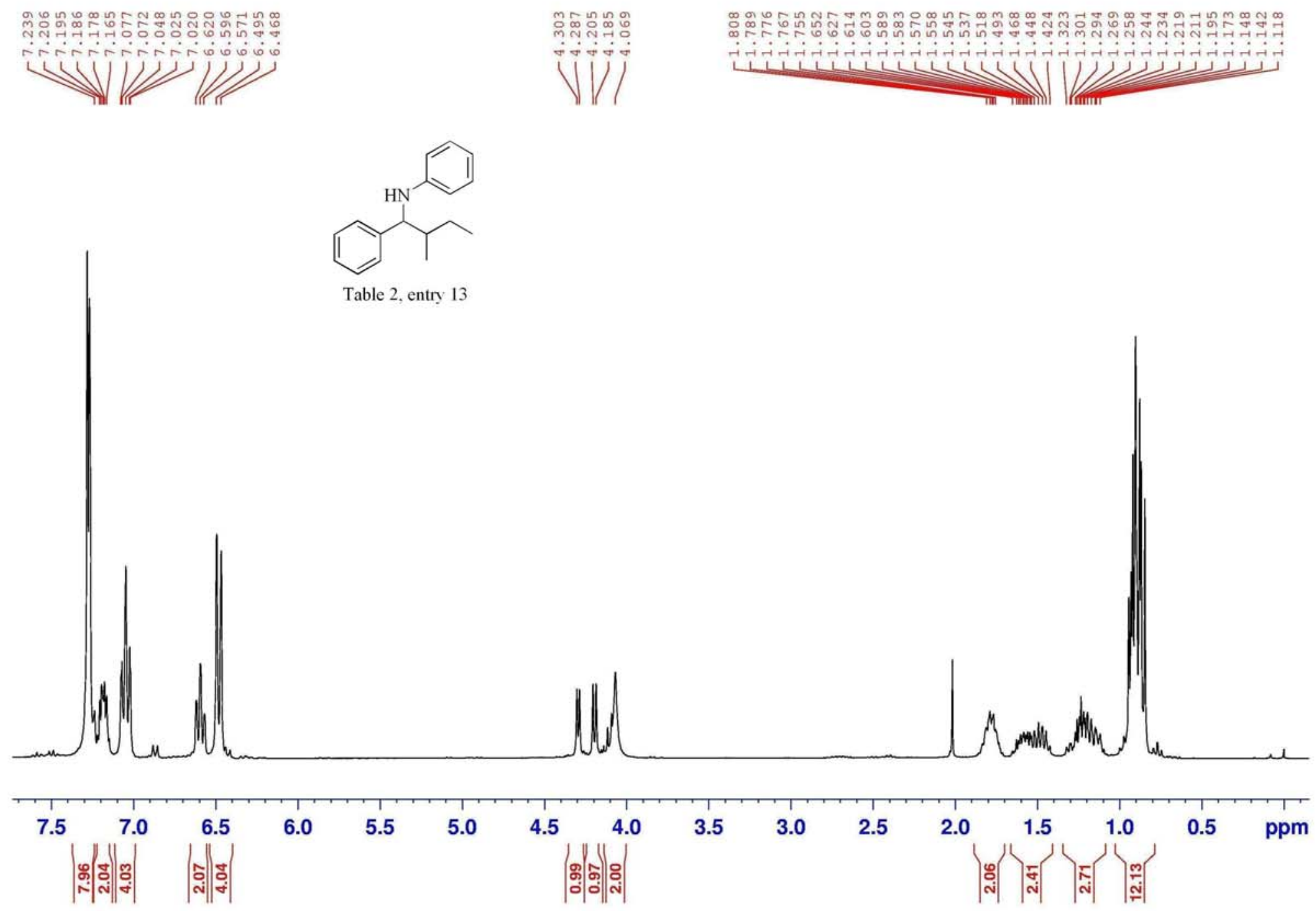

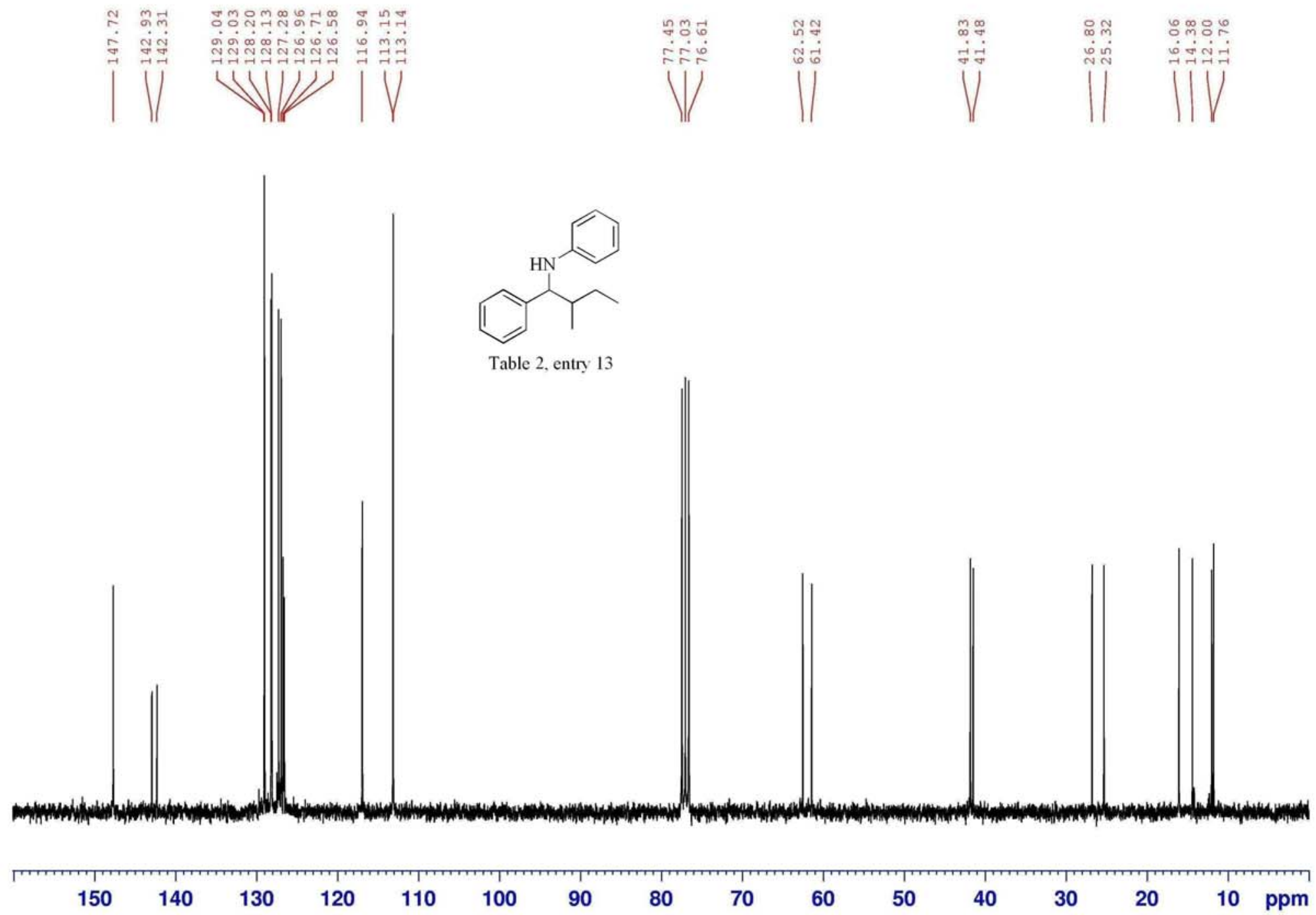


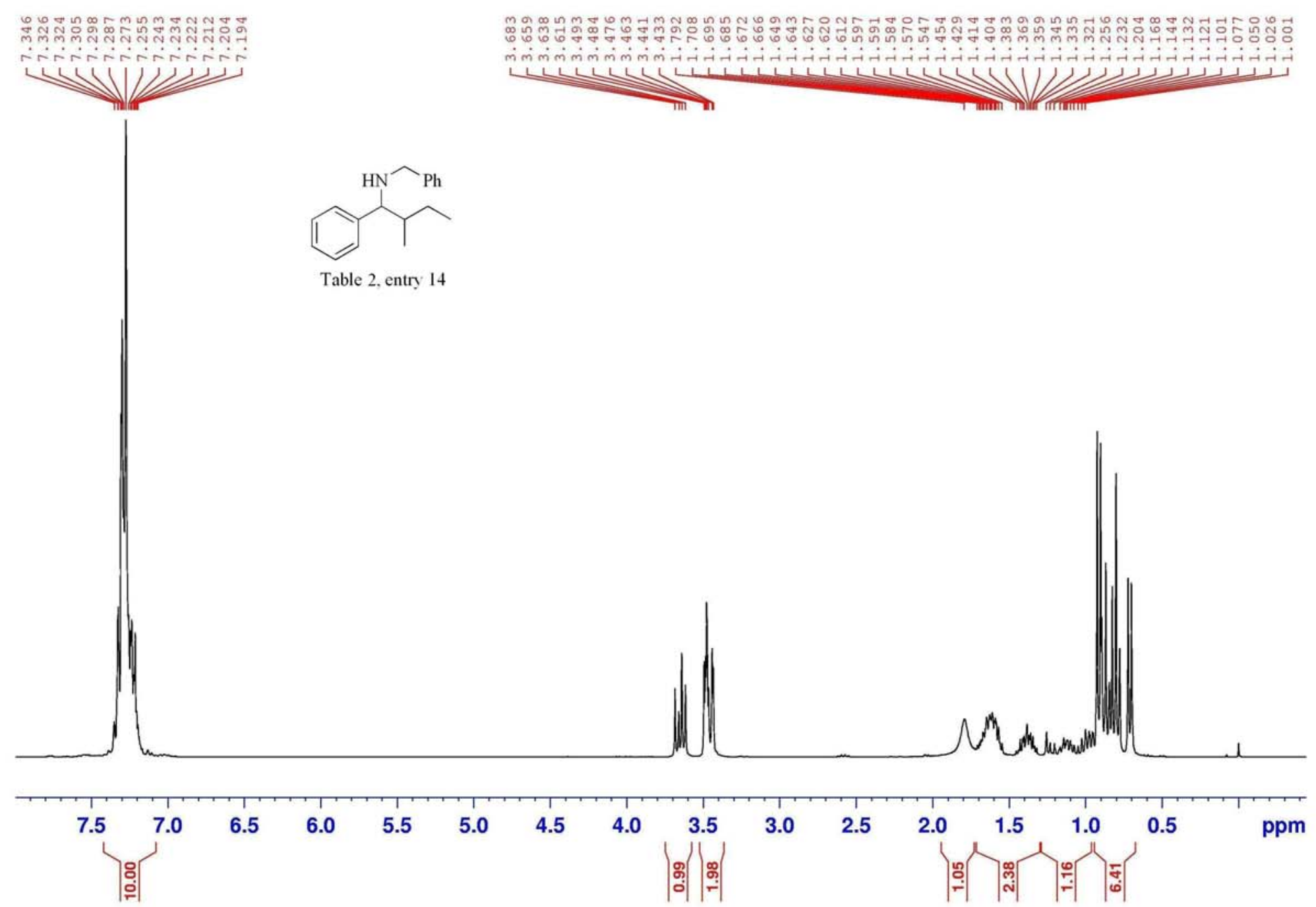




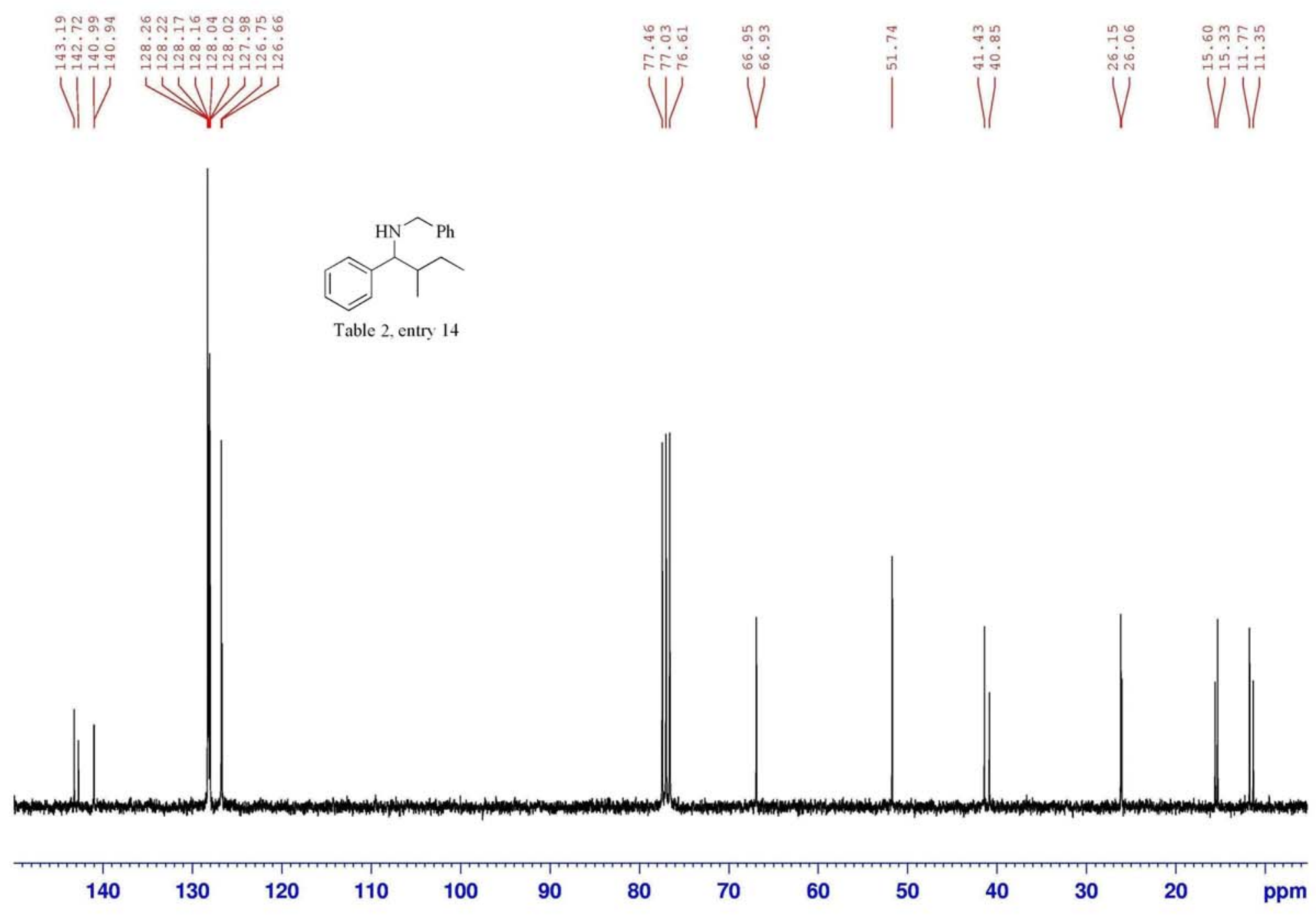




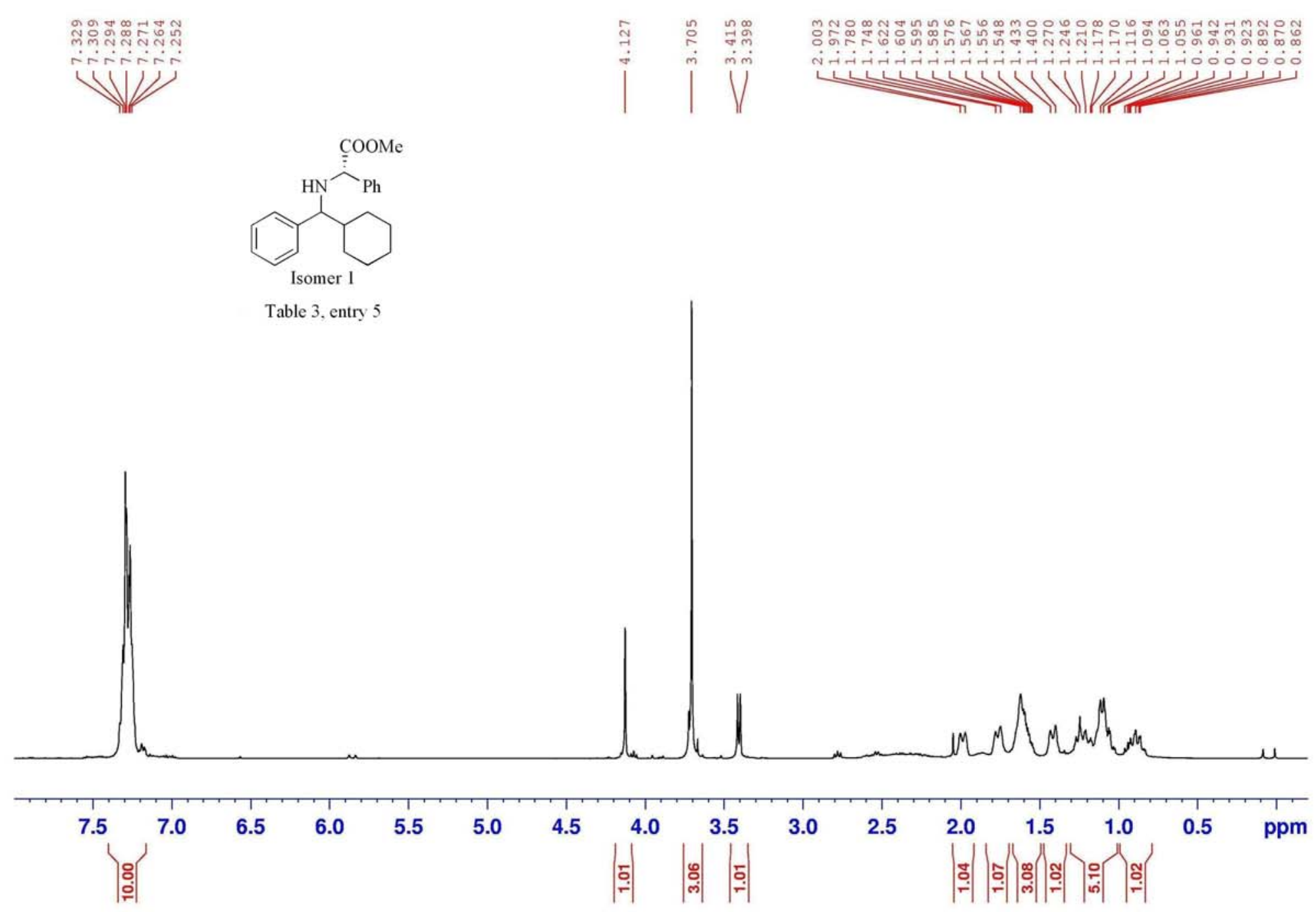




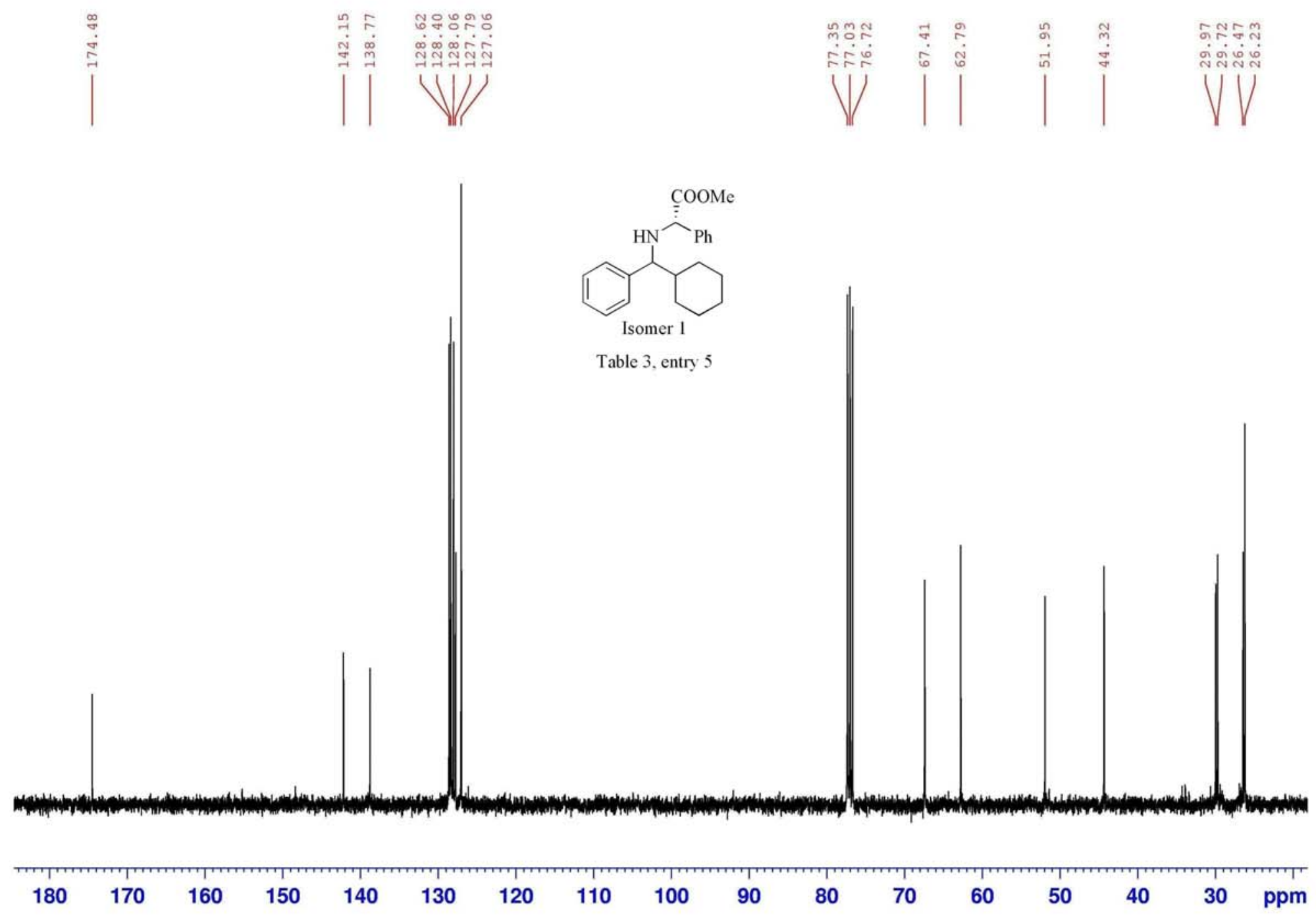



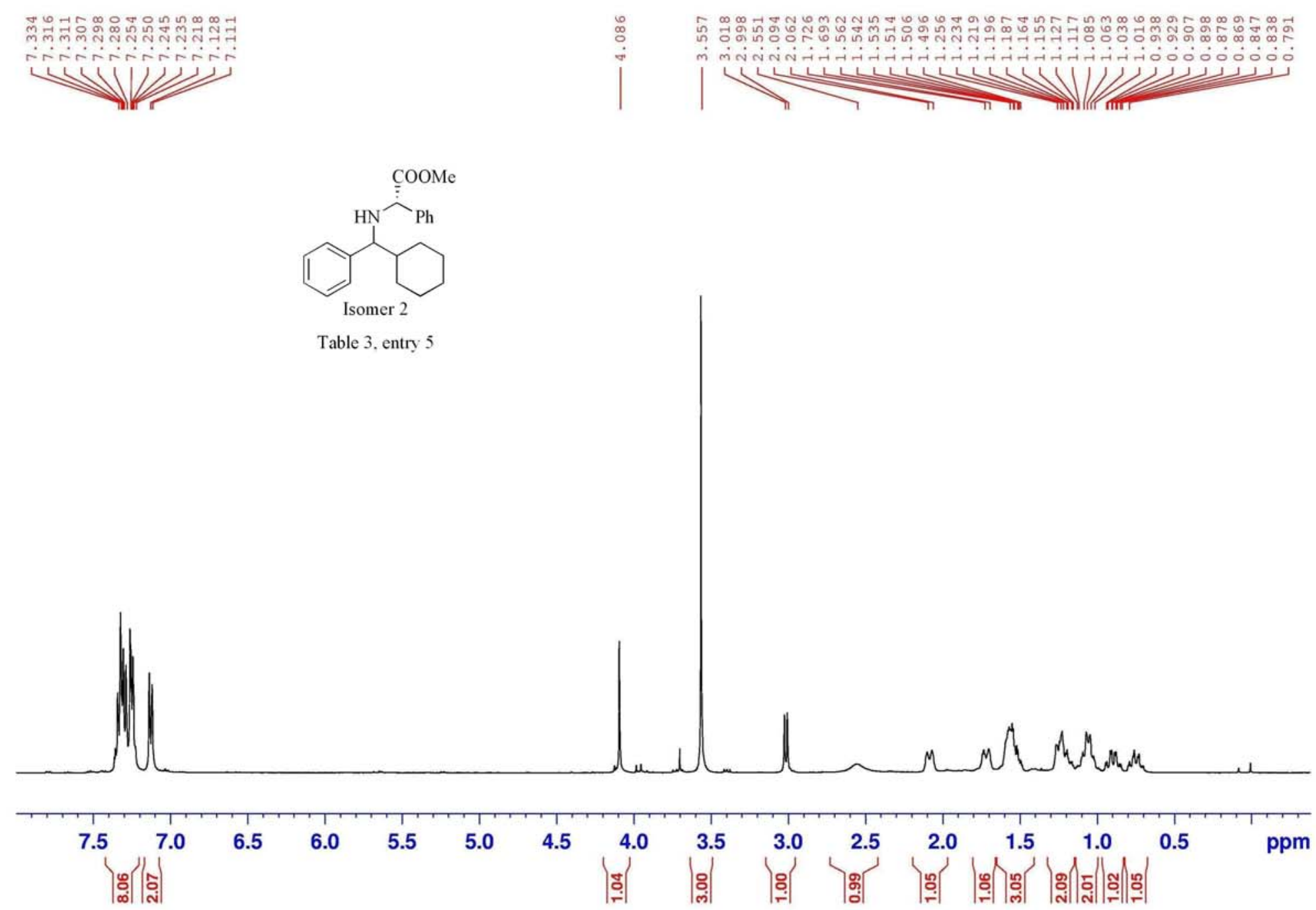


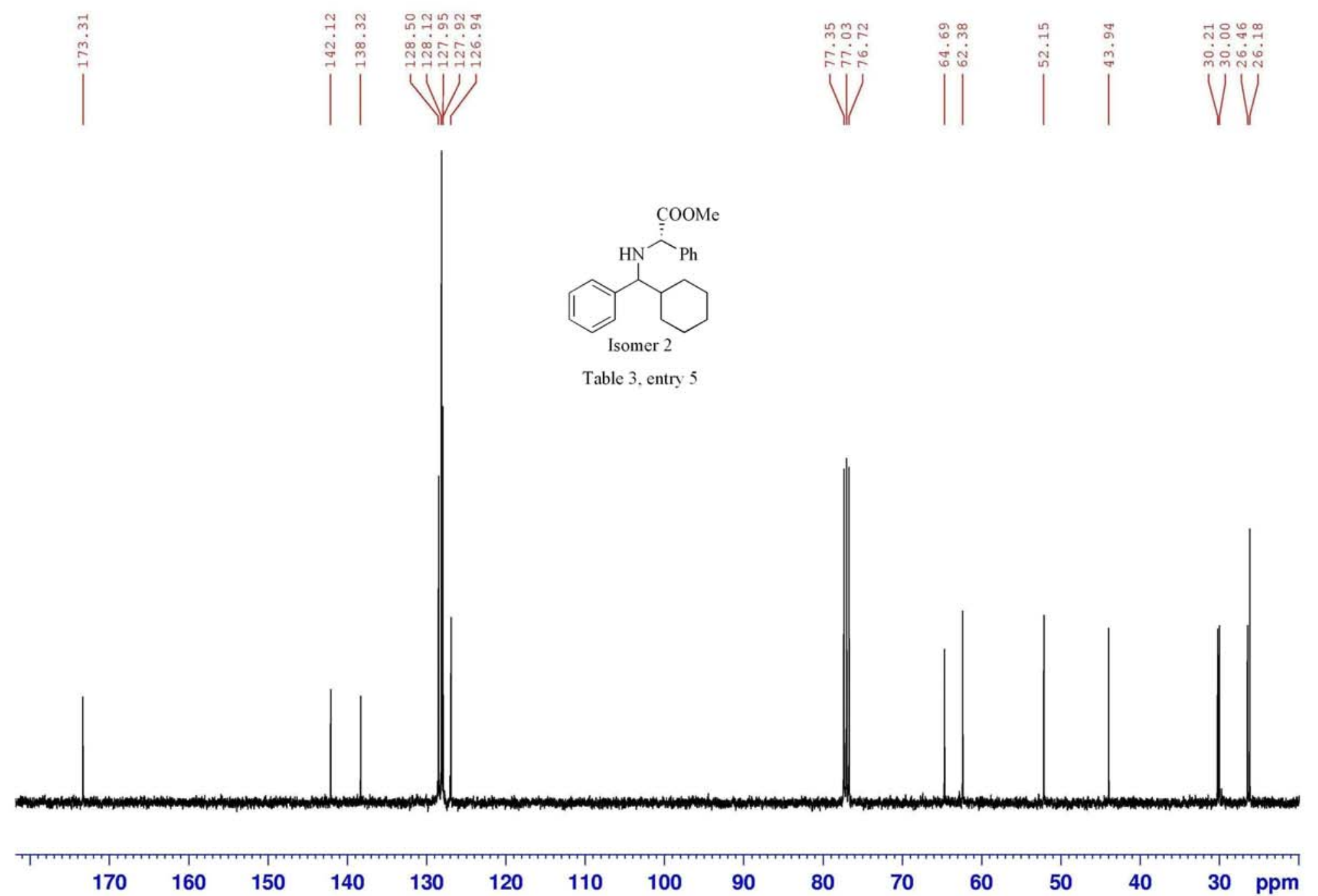




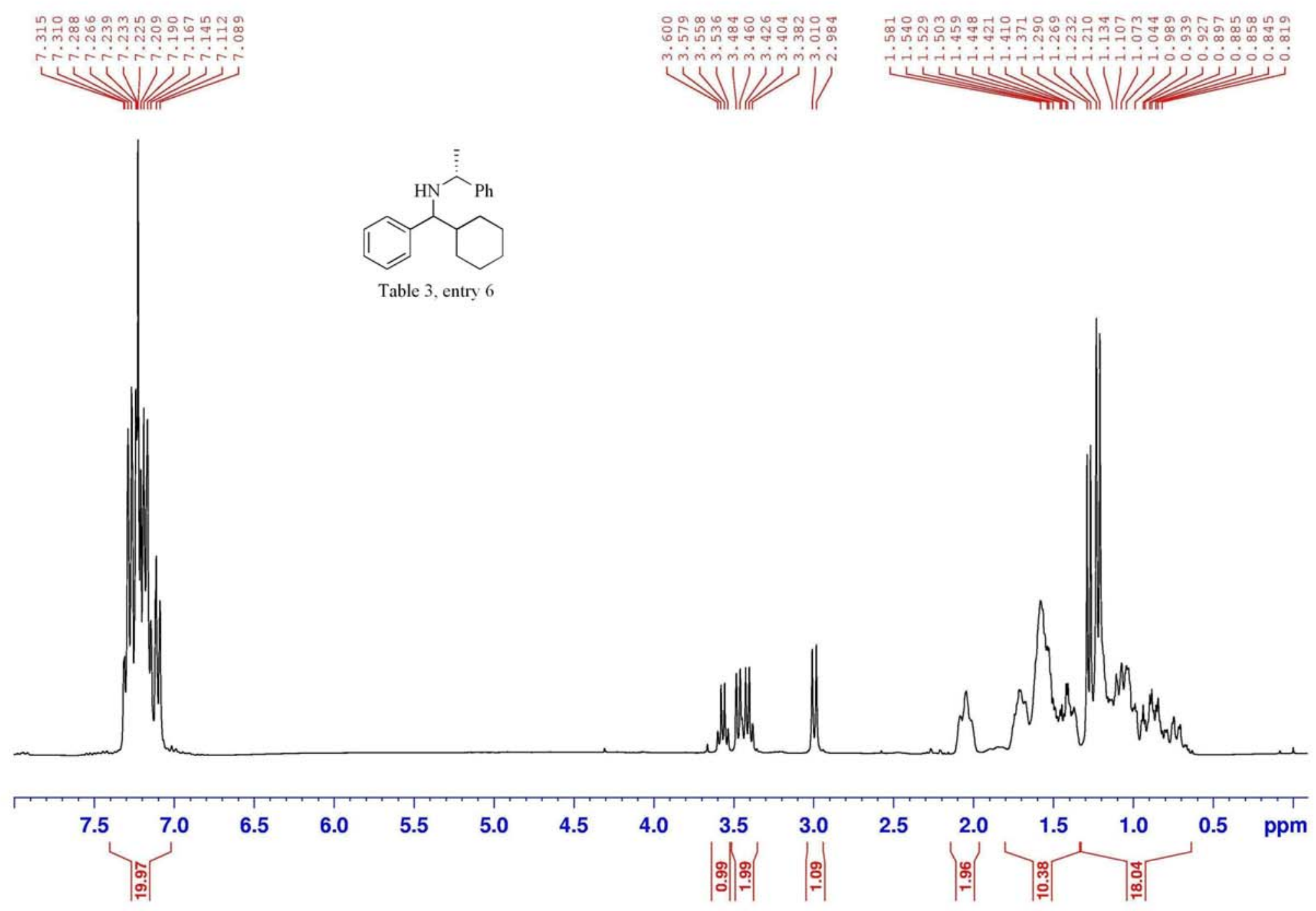




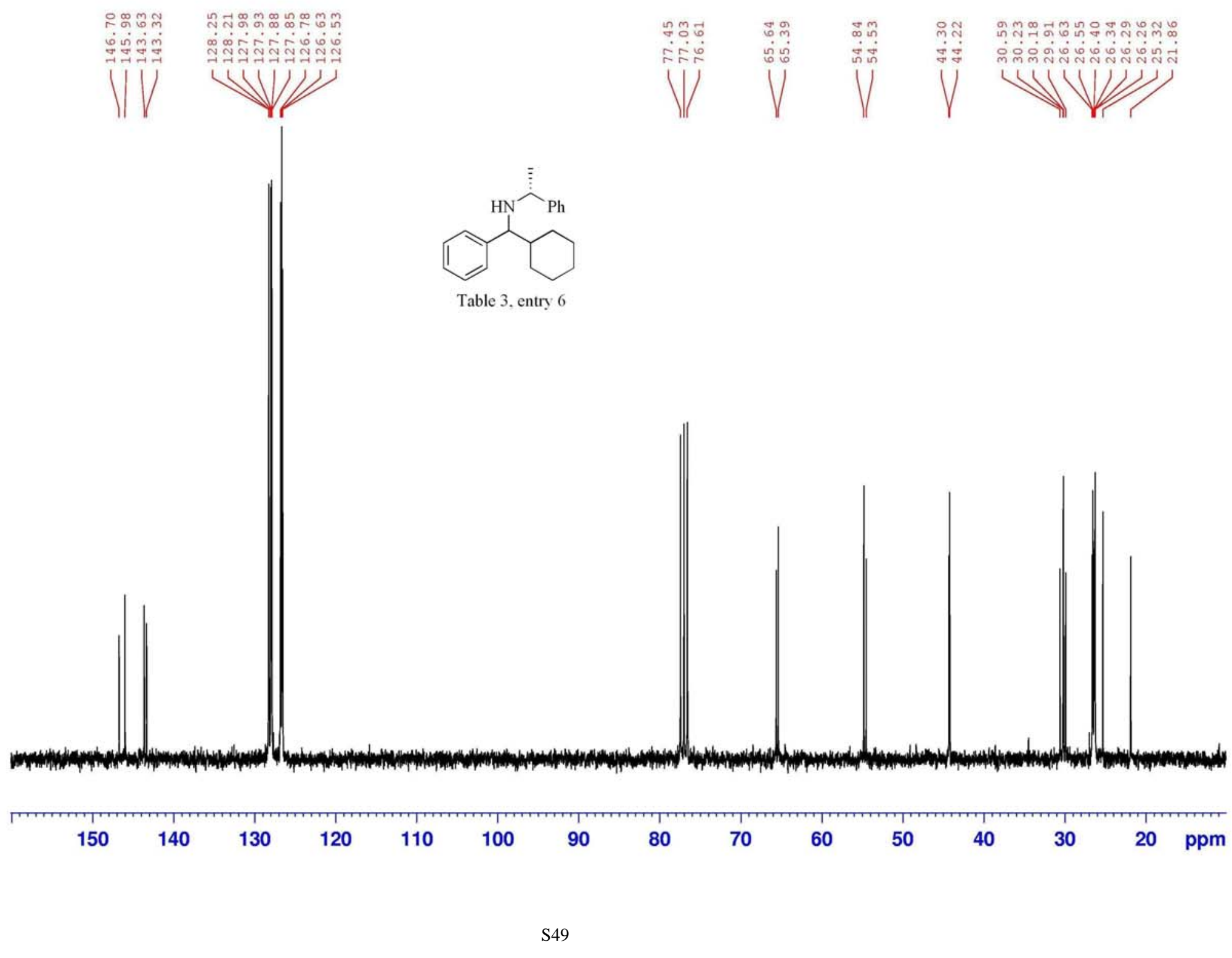




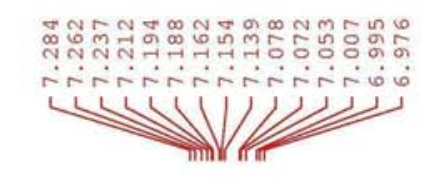

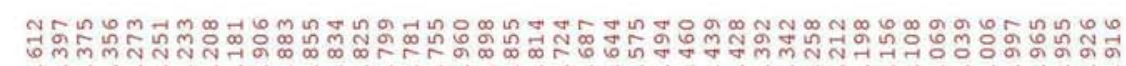
$\longrightarrow+1$
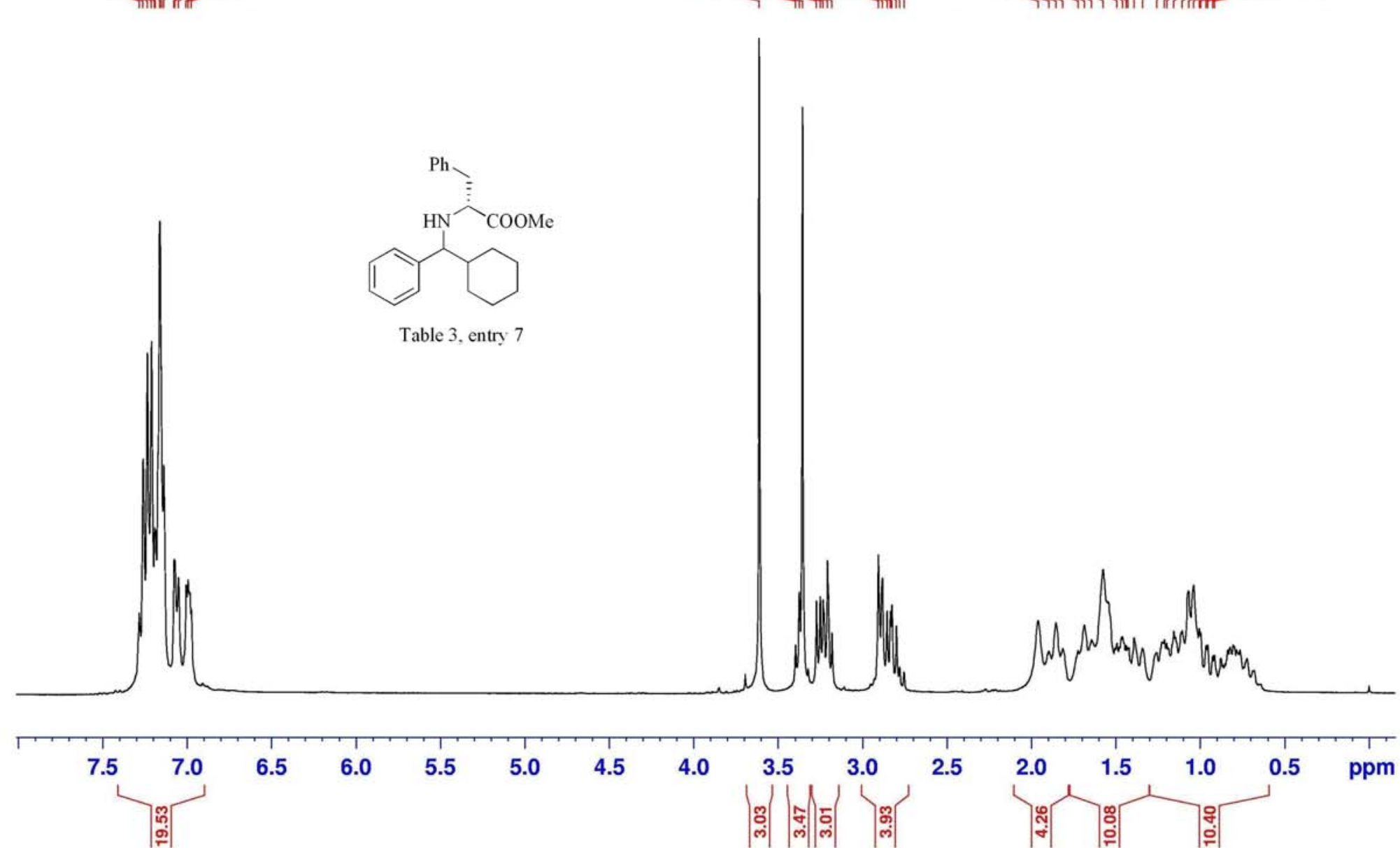


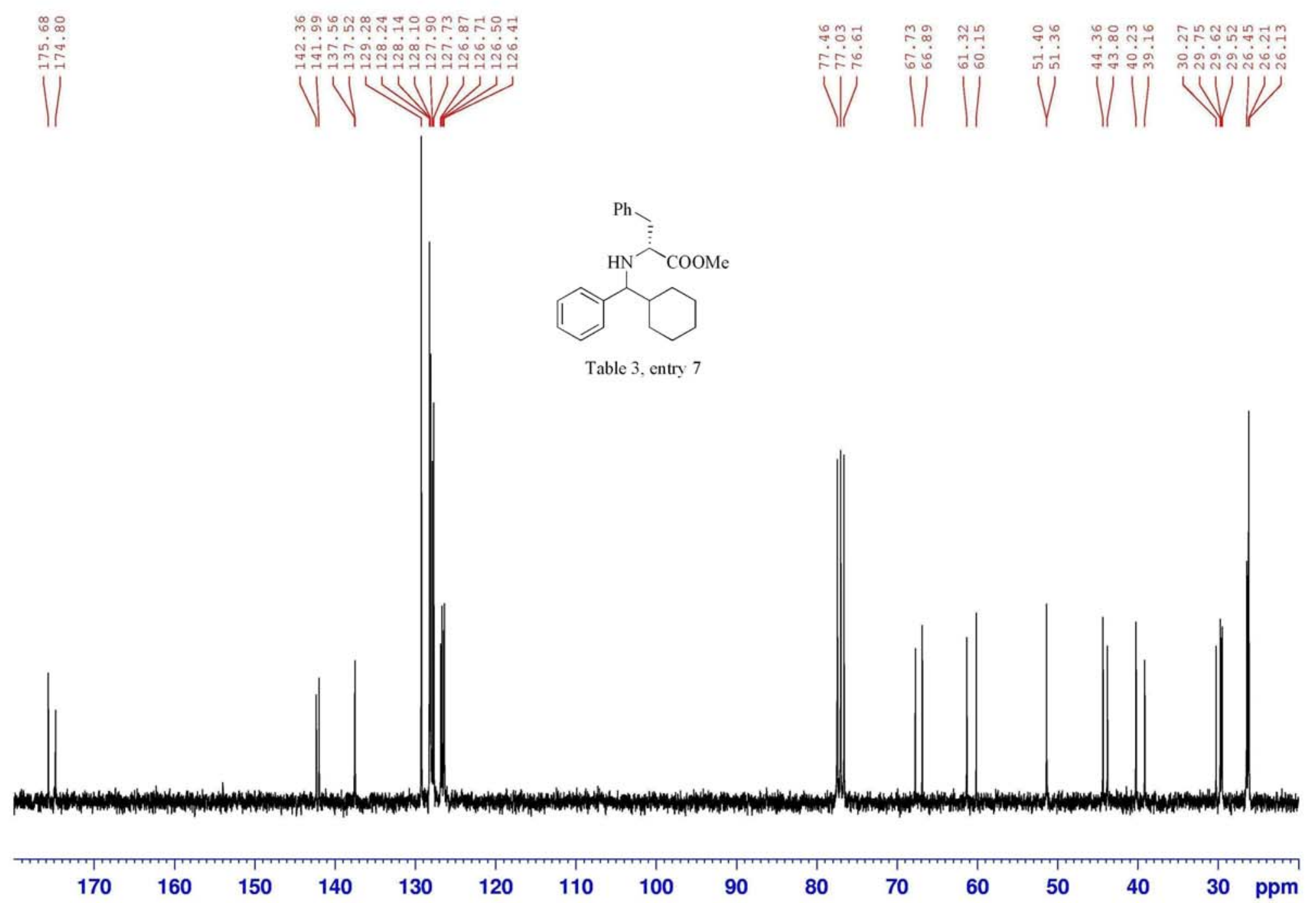




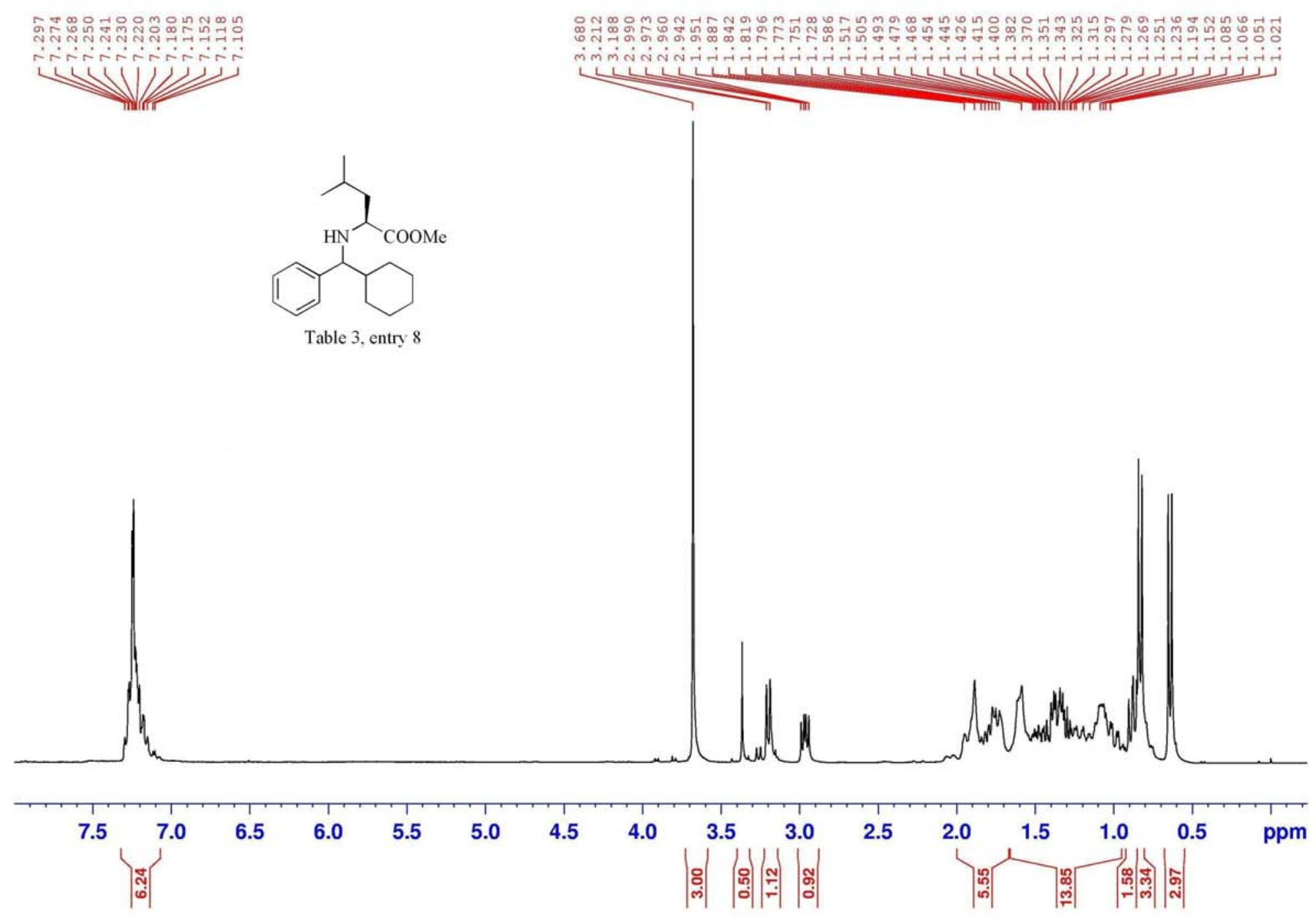




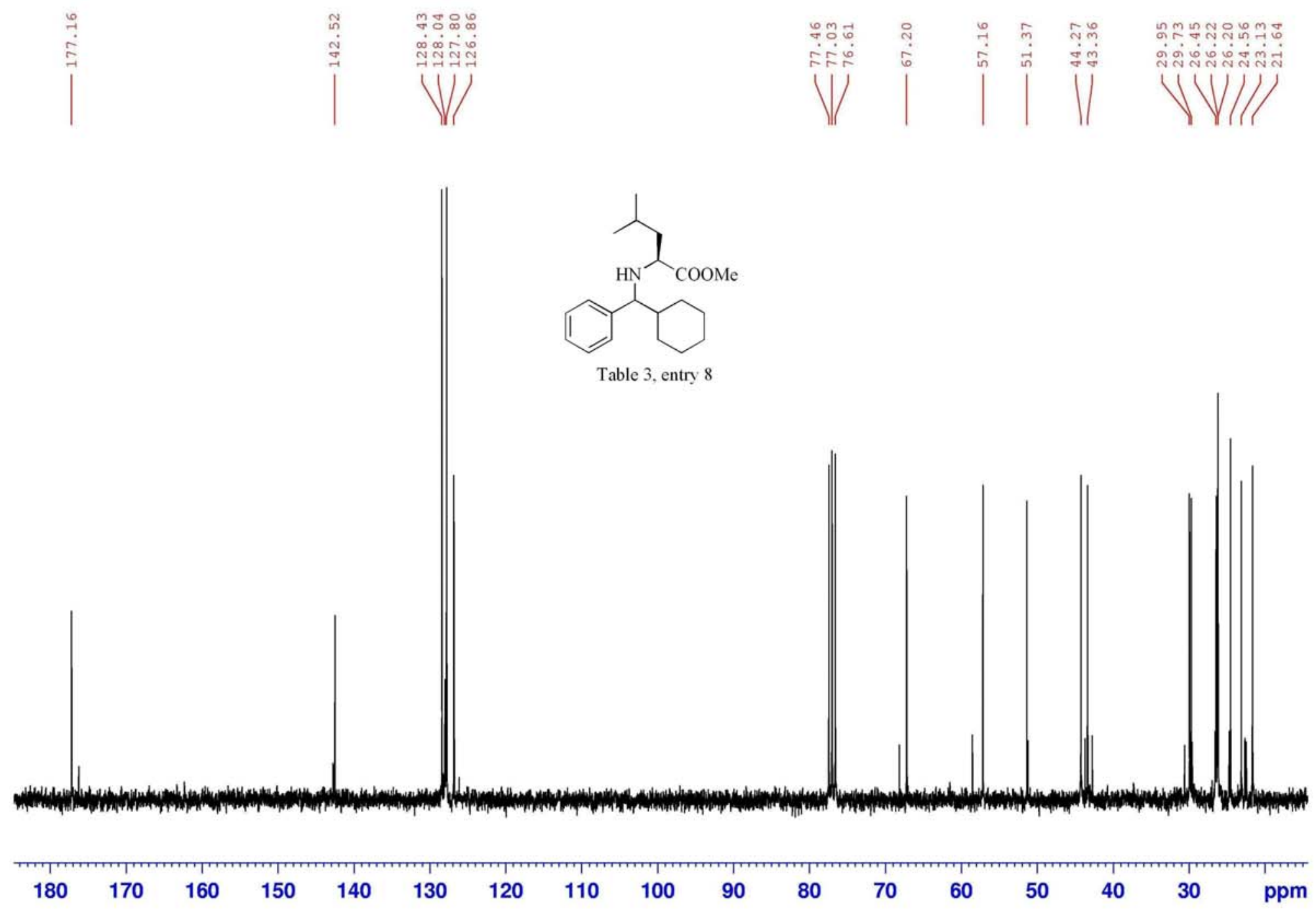




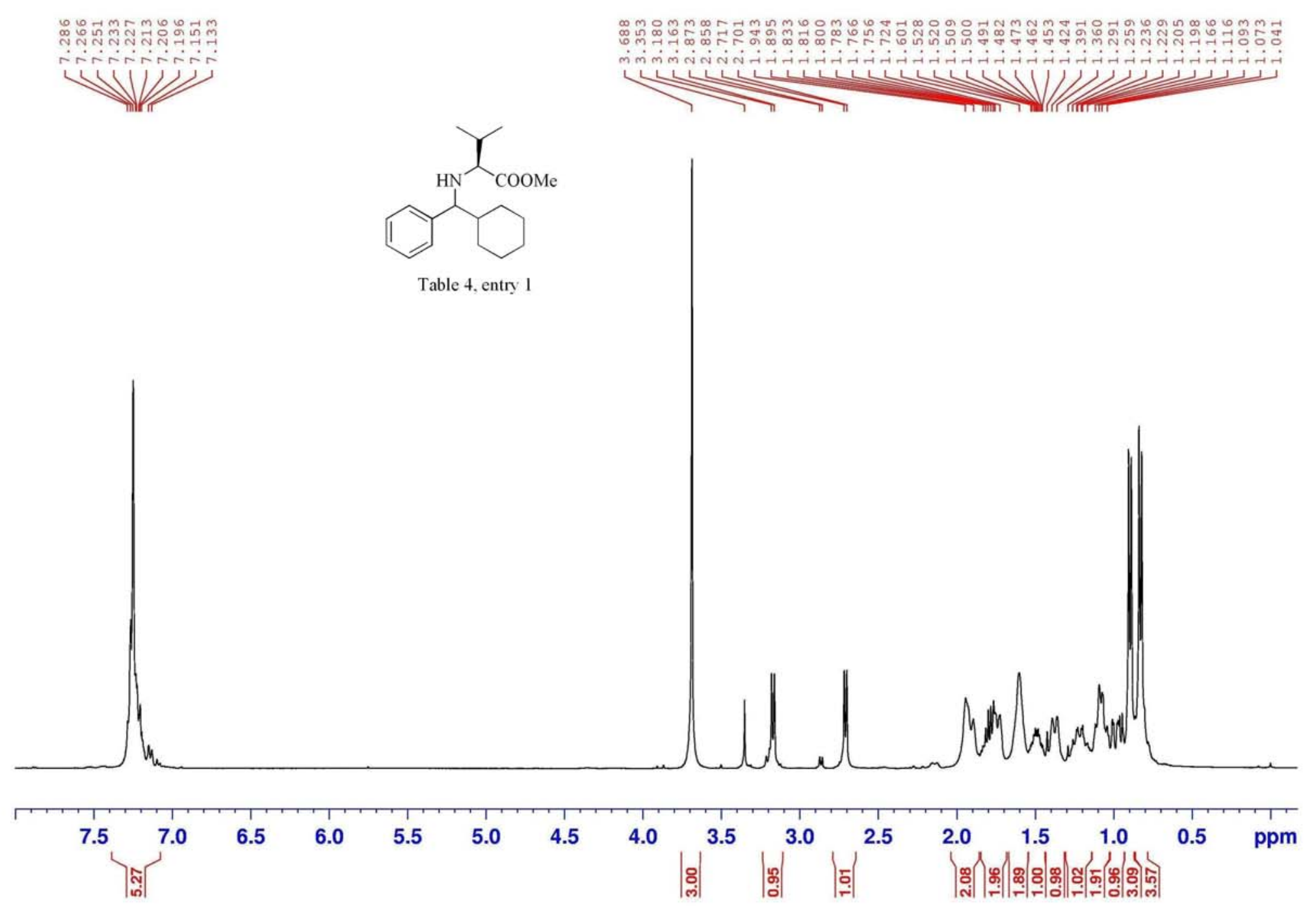




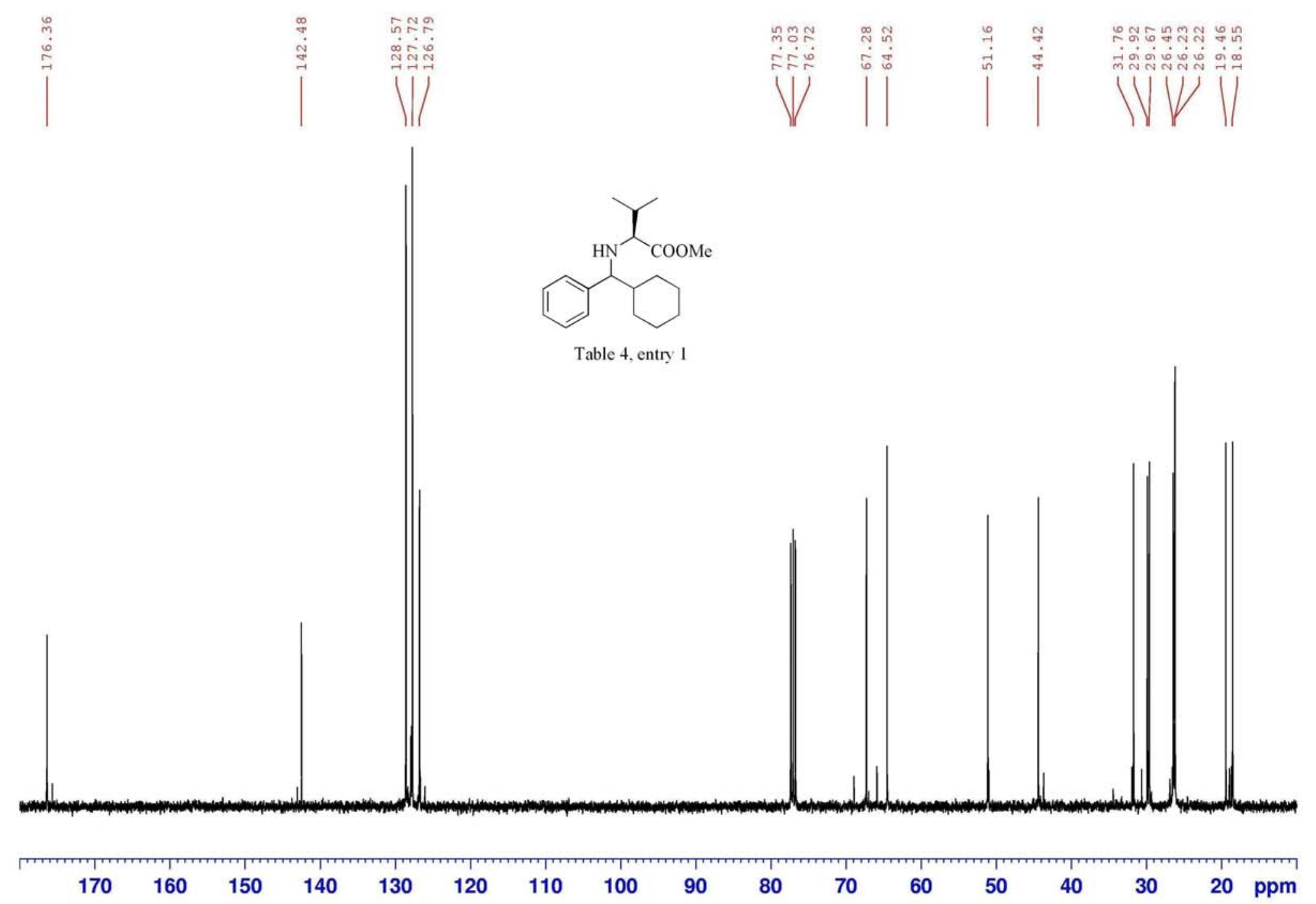




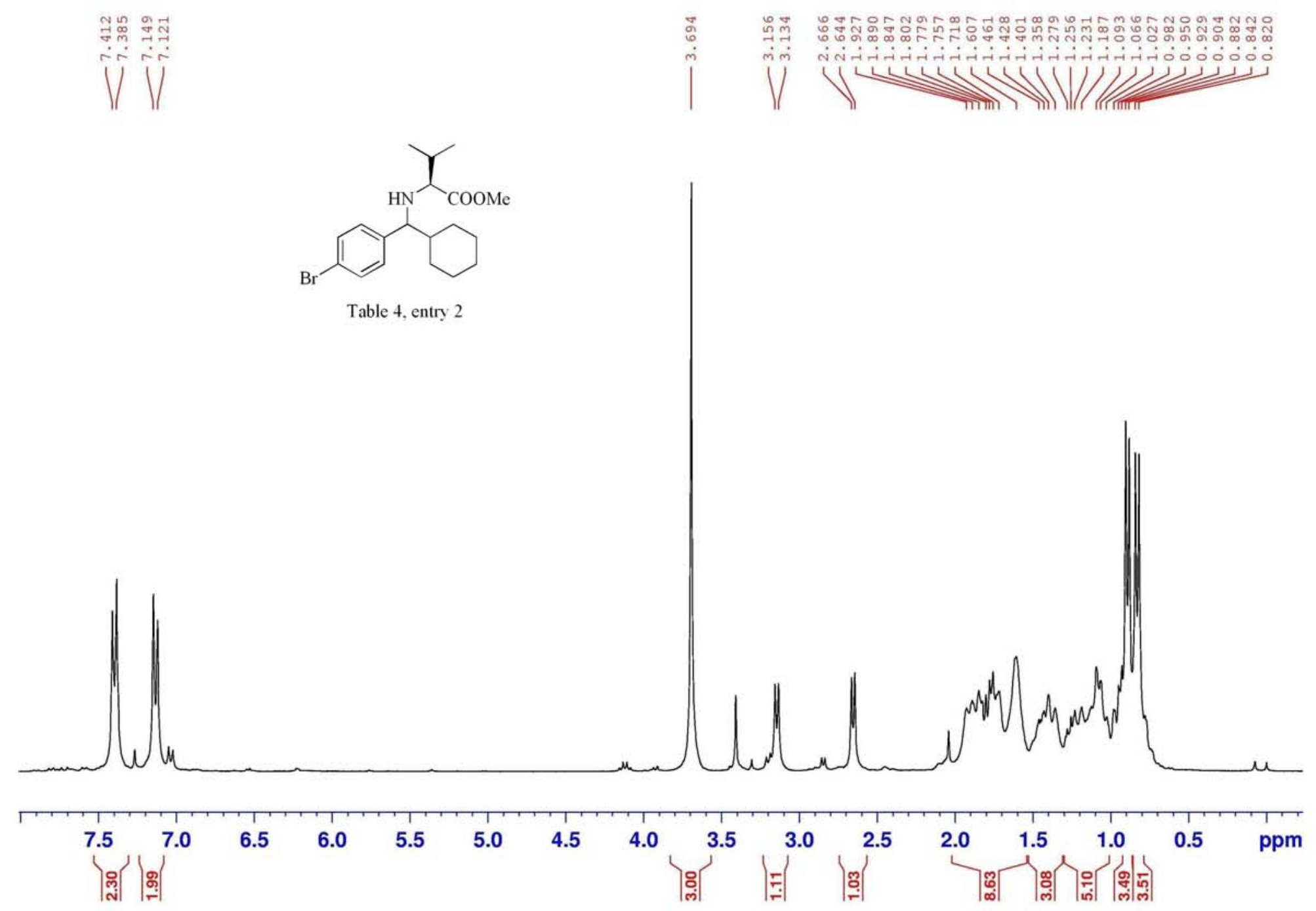




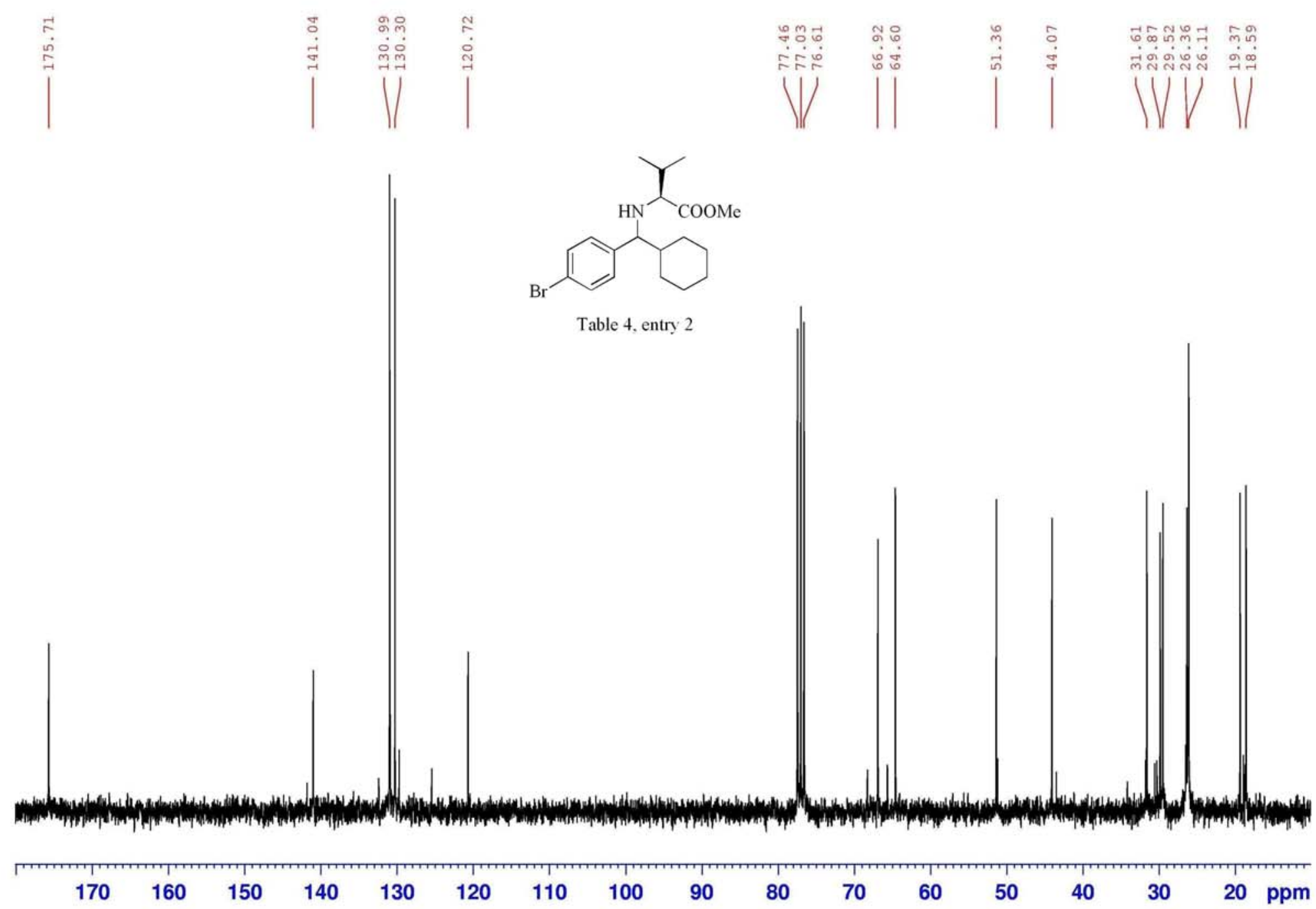




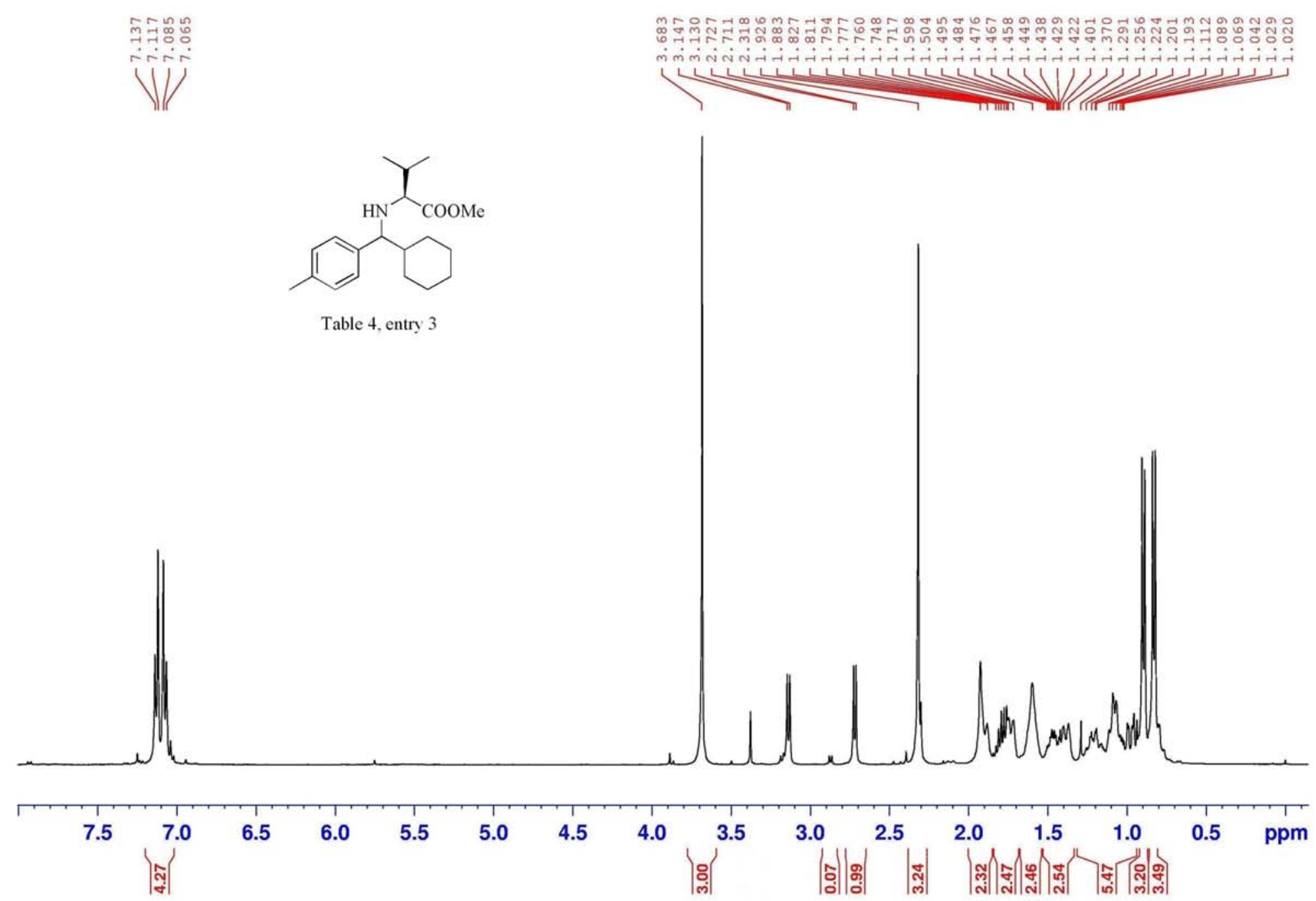




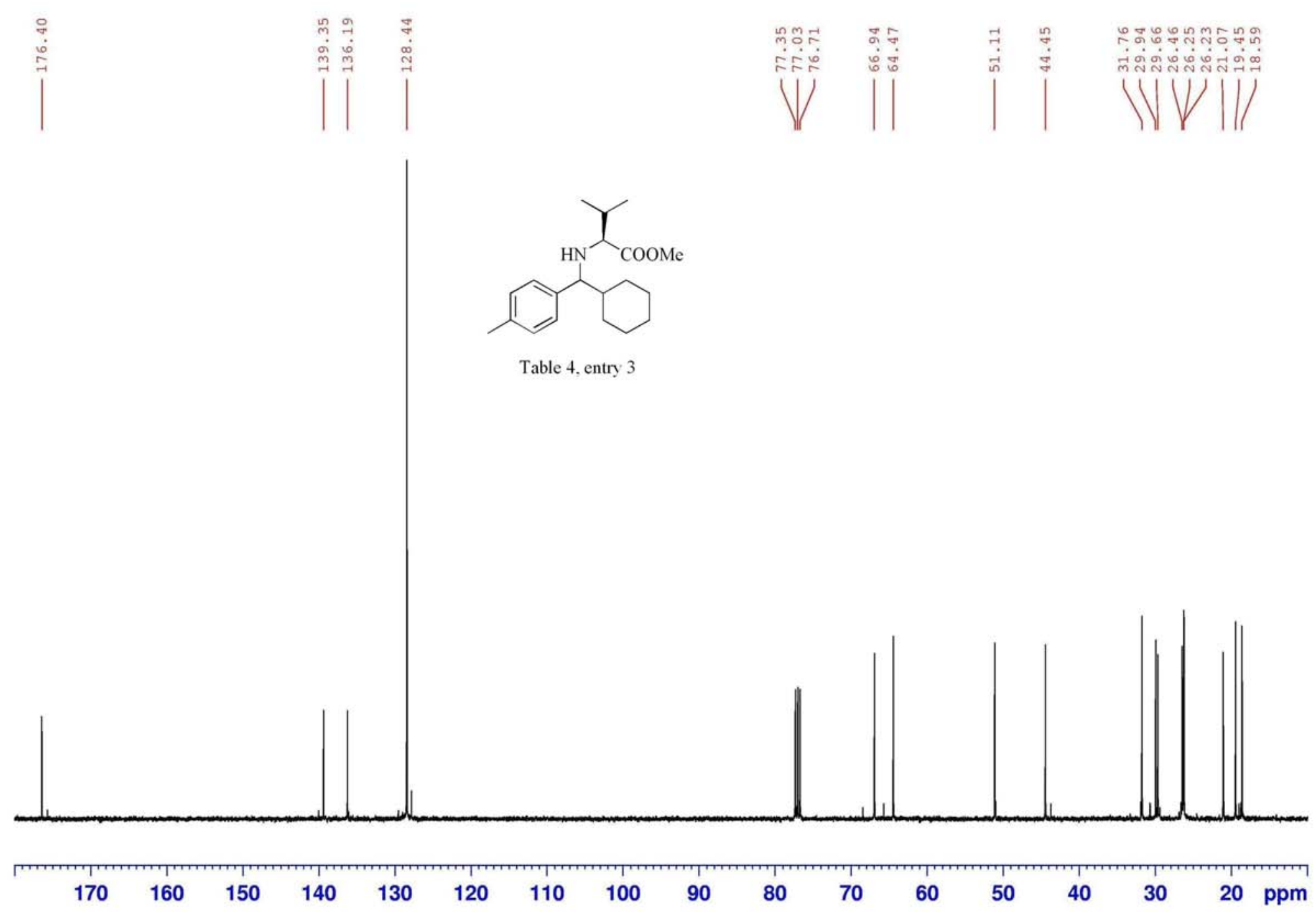




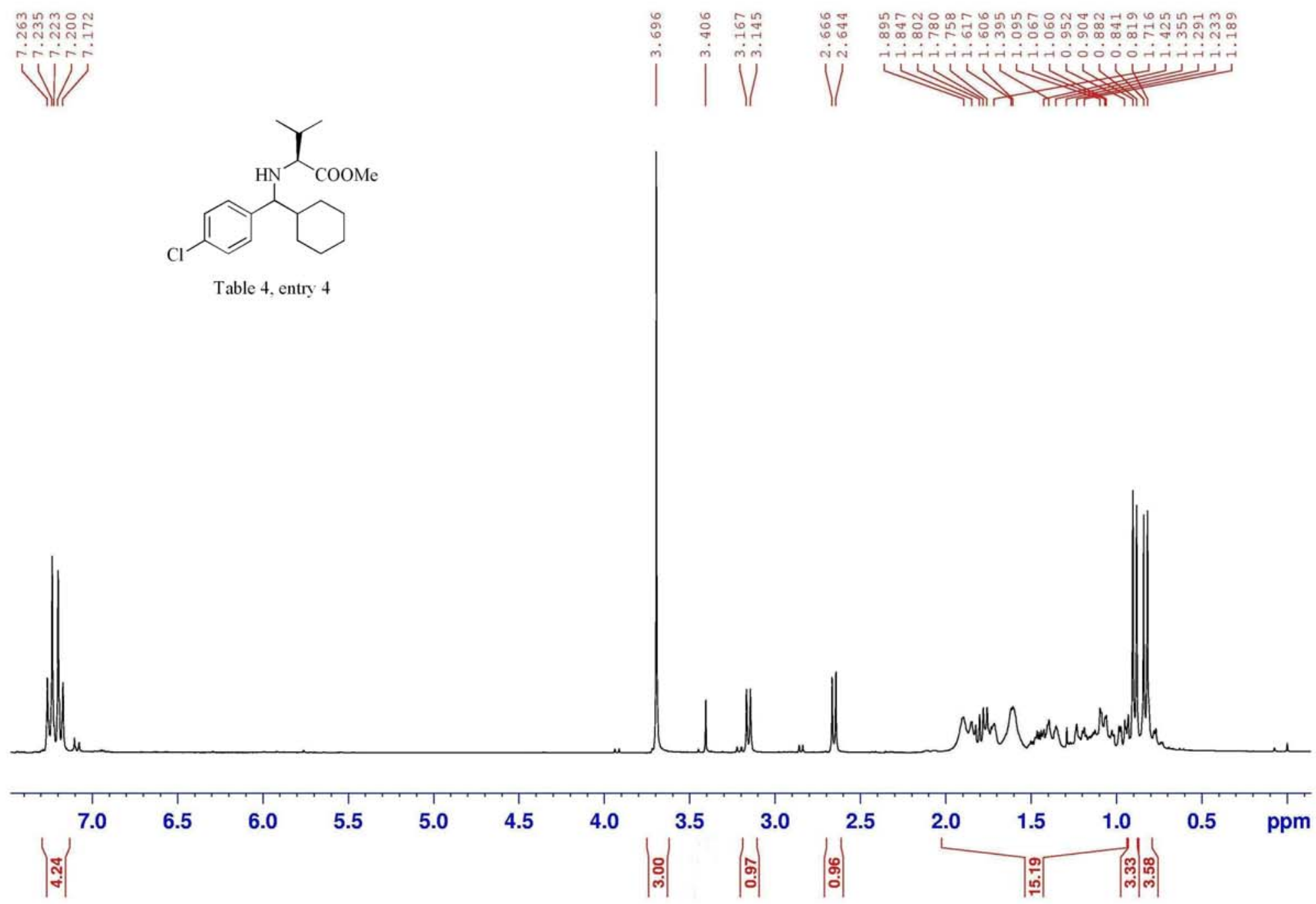




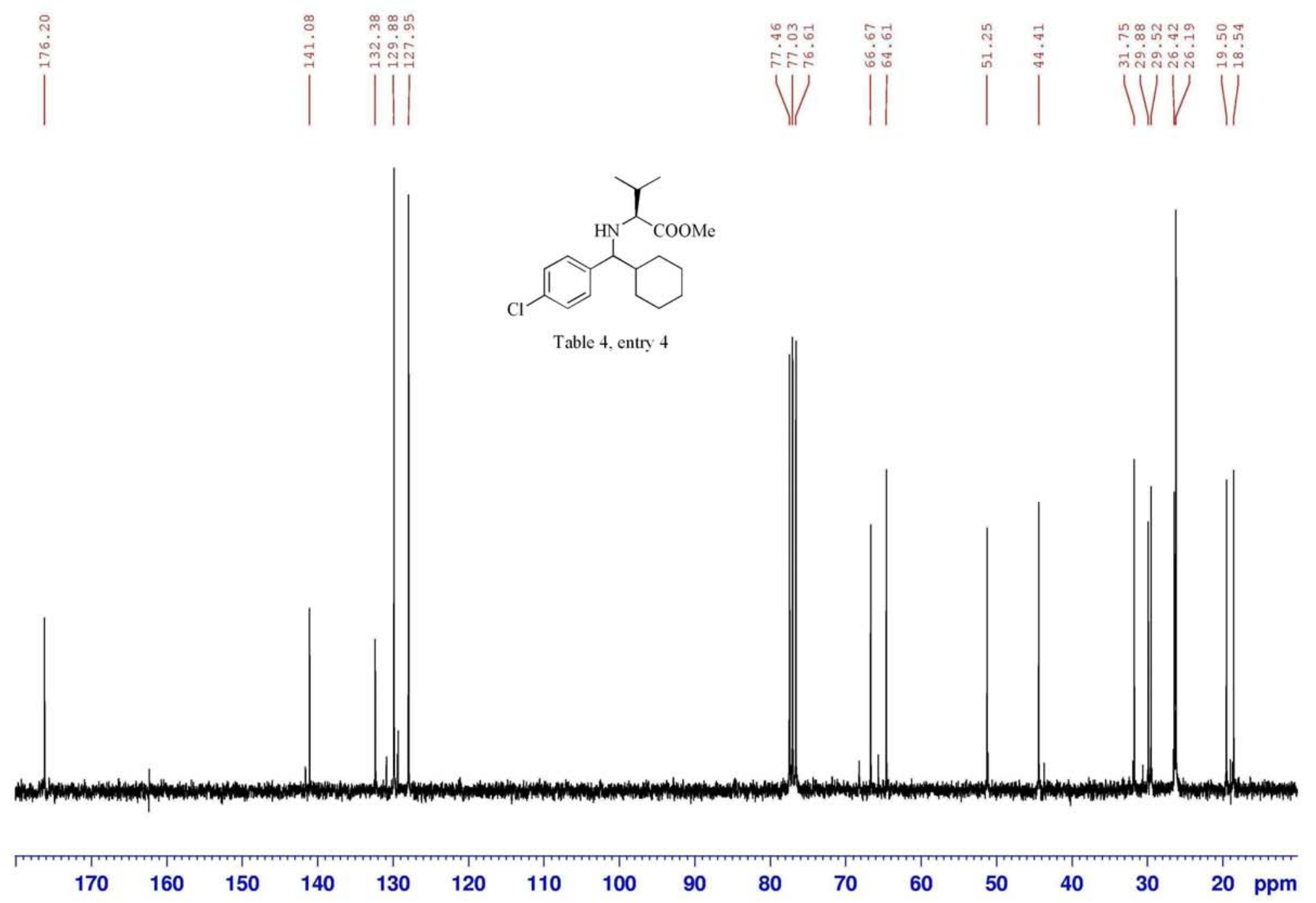




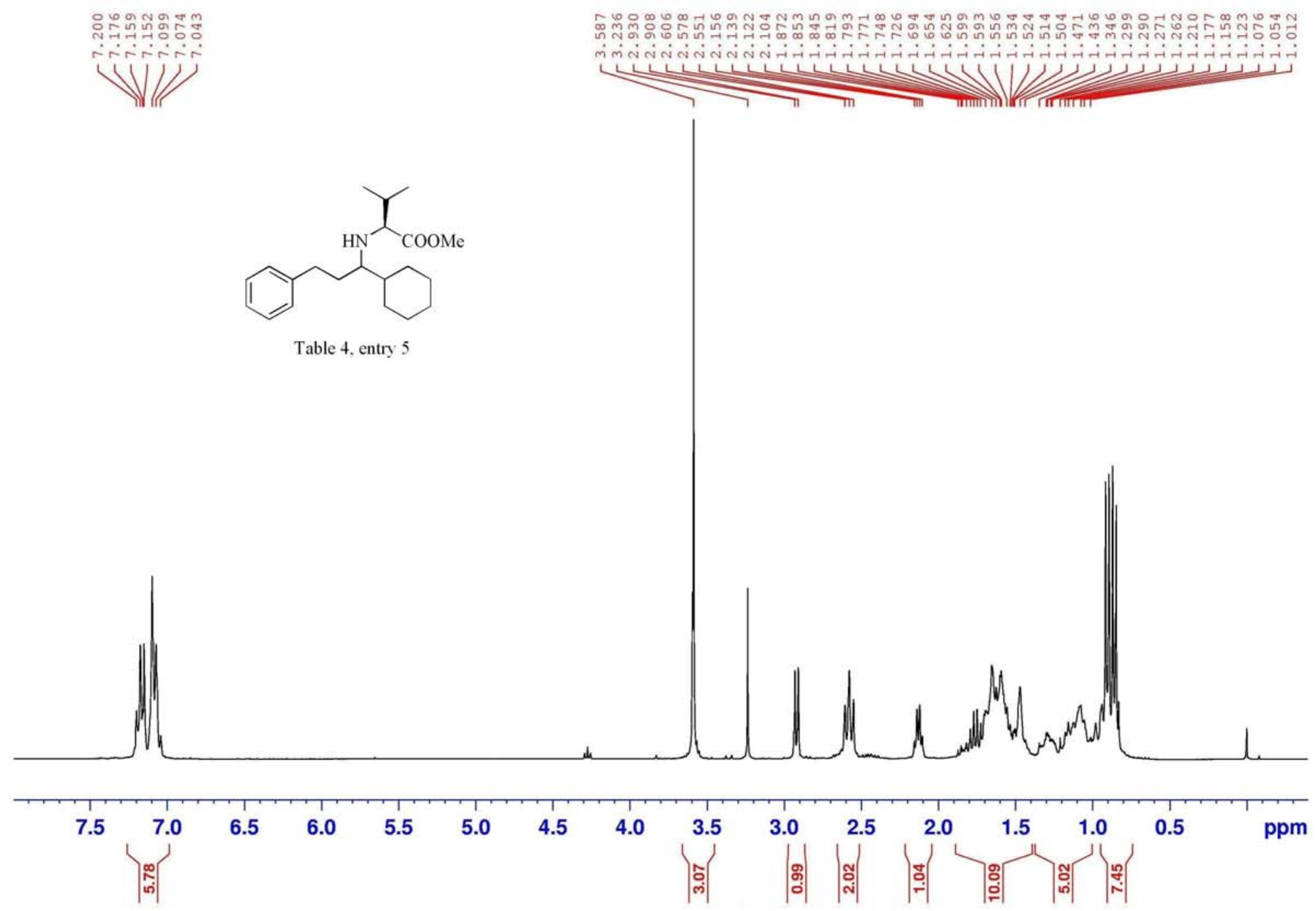




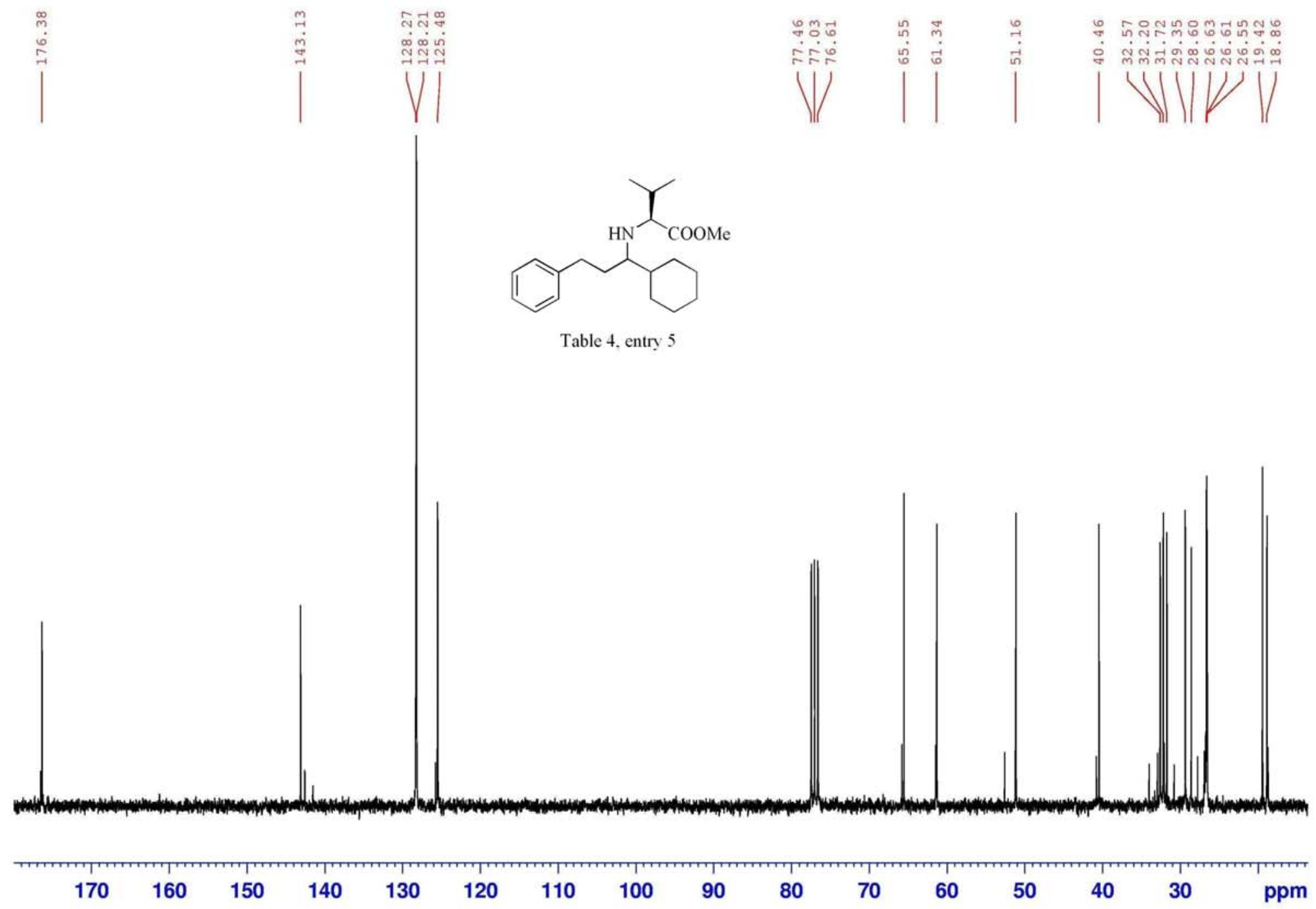




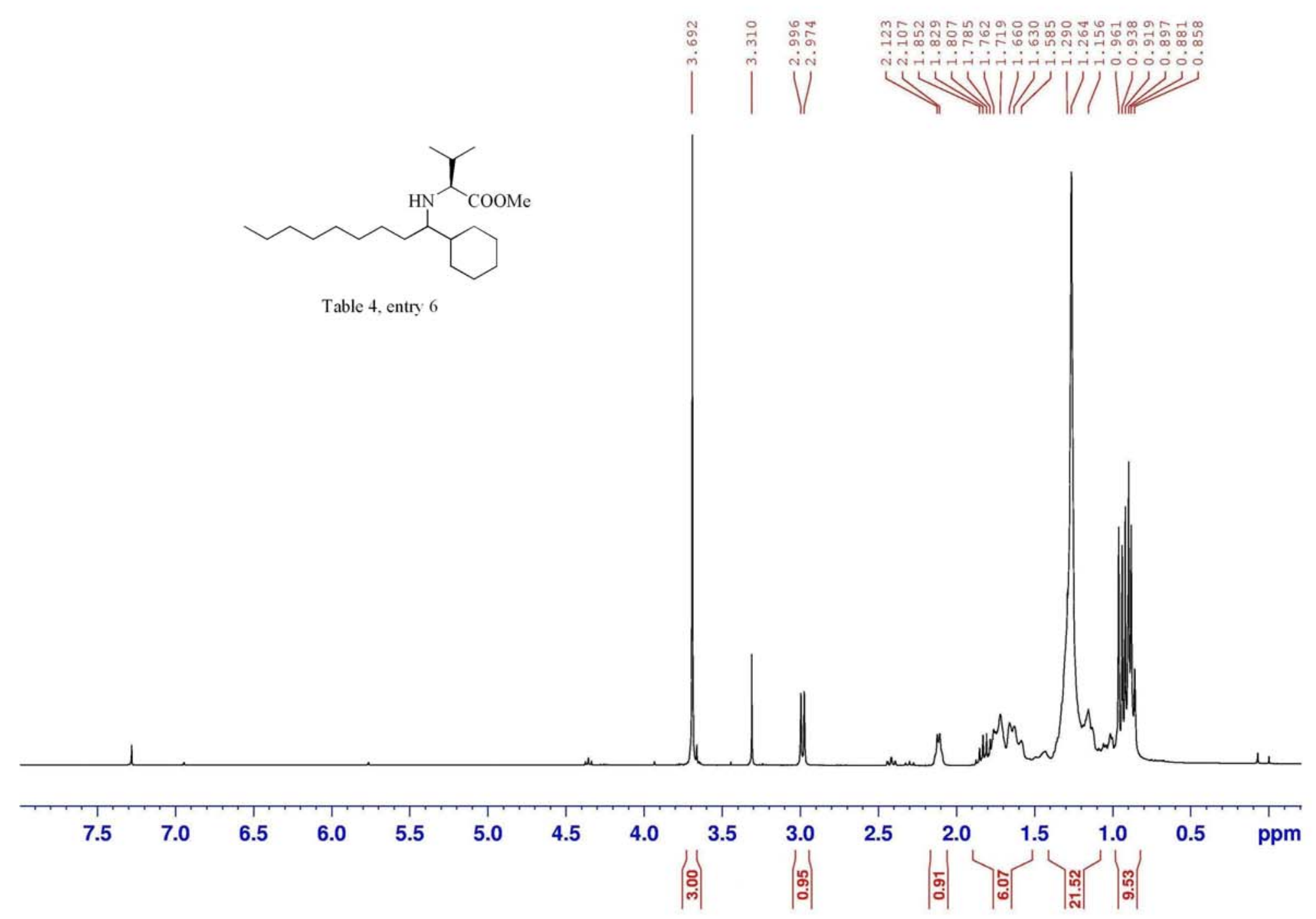




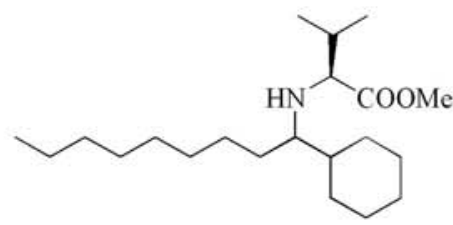

Table 4, entry 6
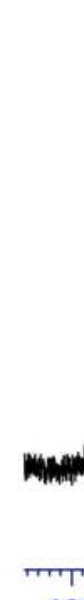

180

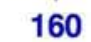

150

140

130

120

110

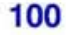

90

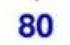

70

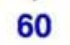

50 

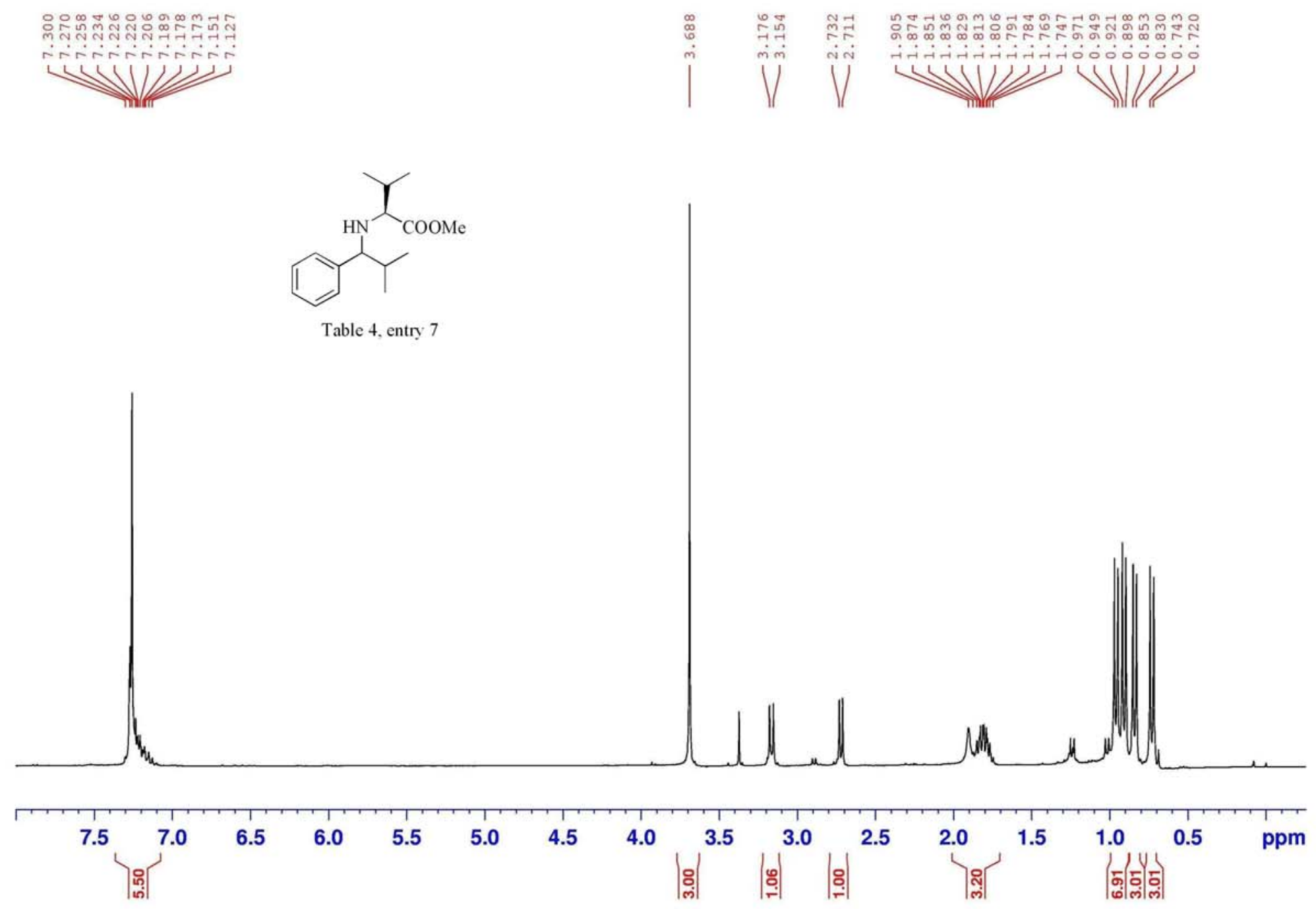


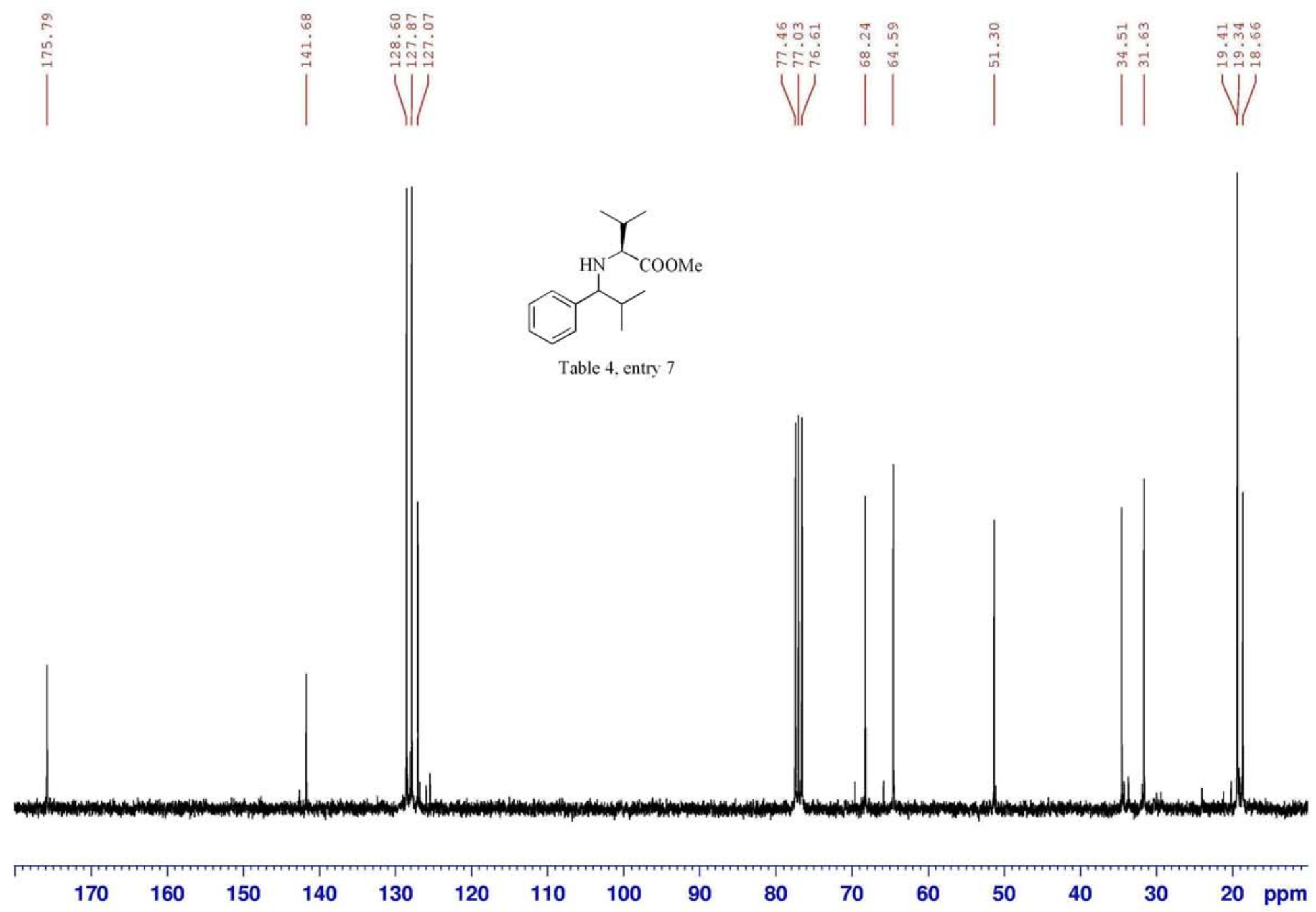




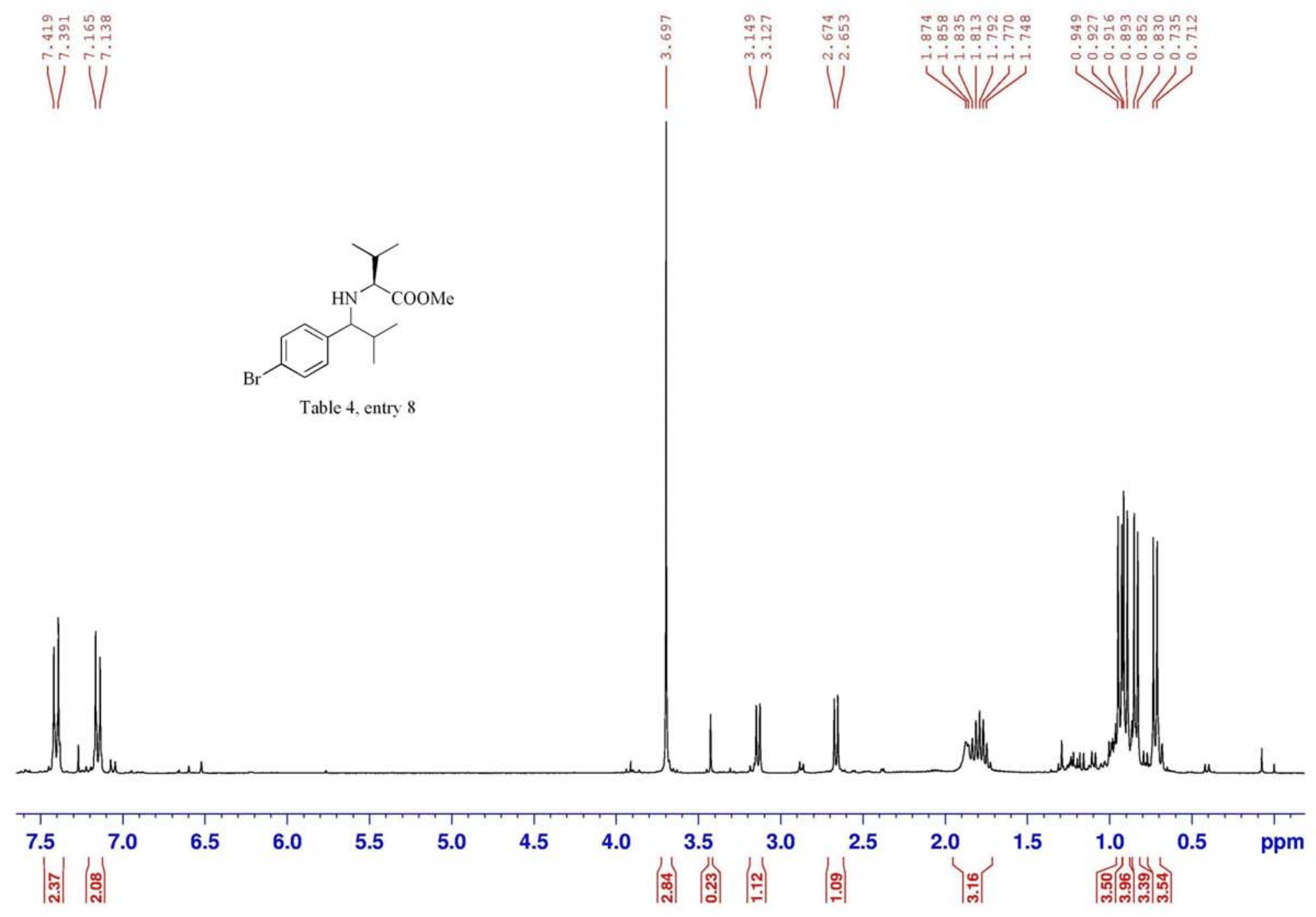



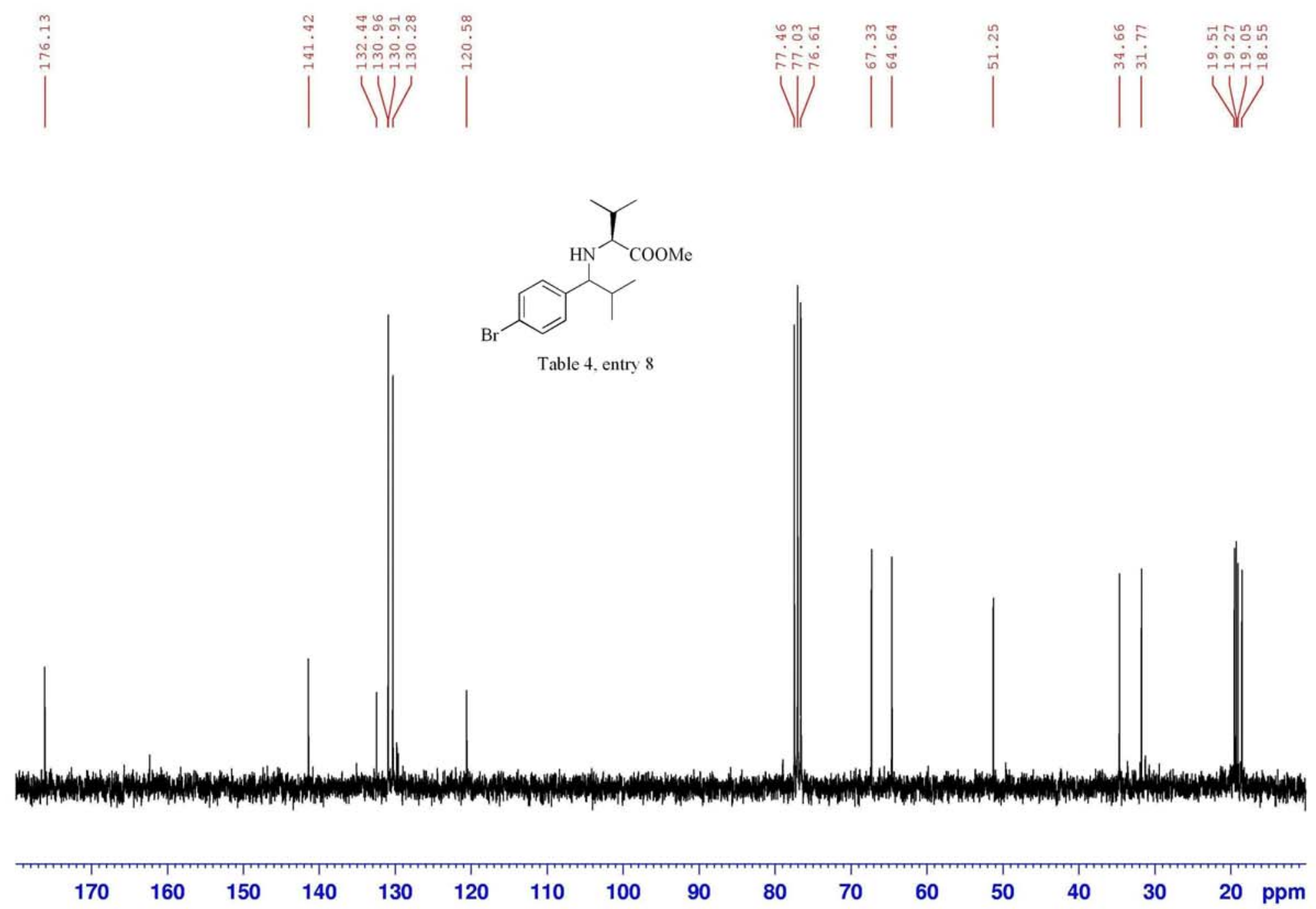


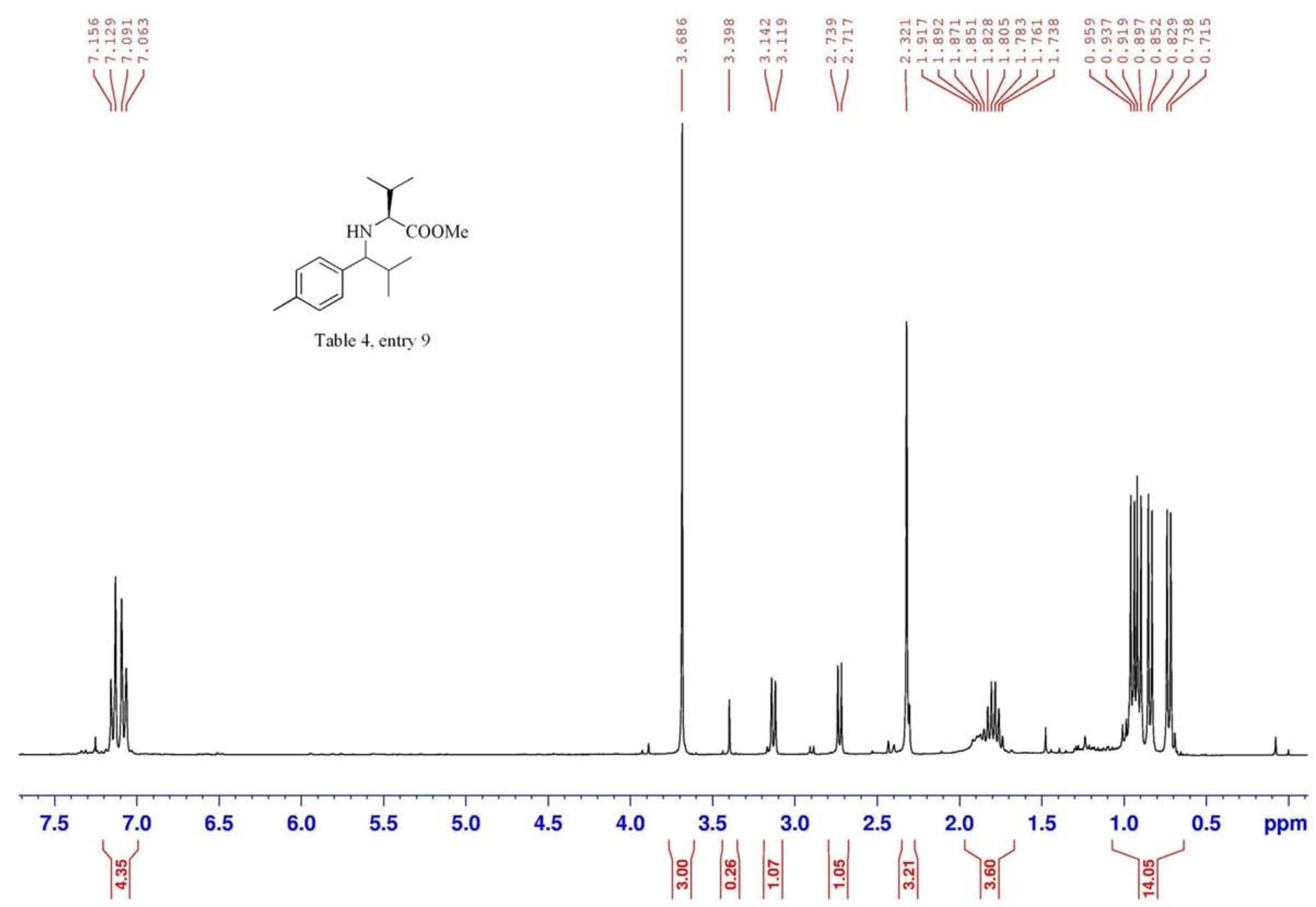




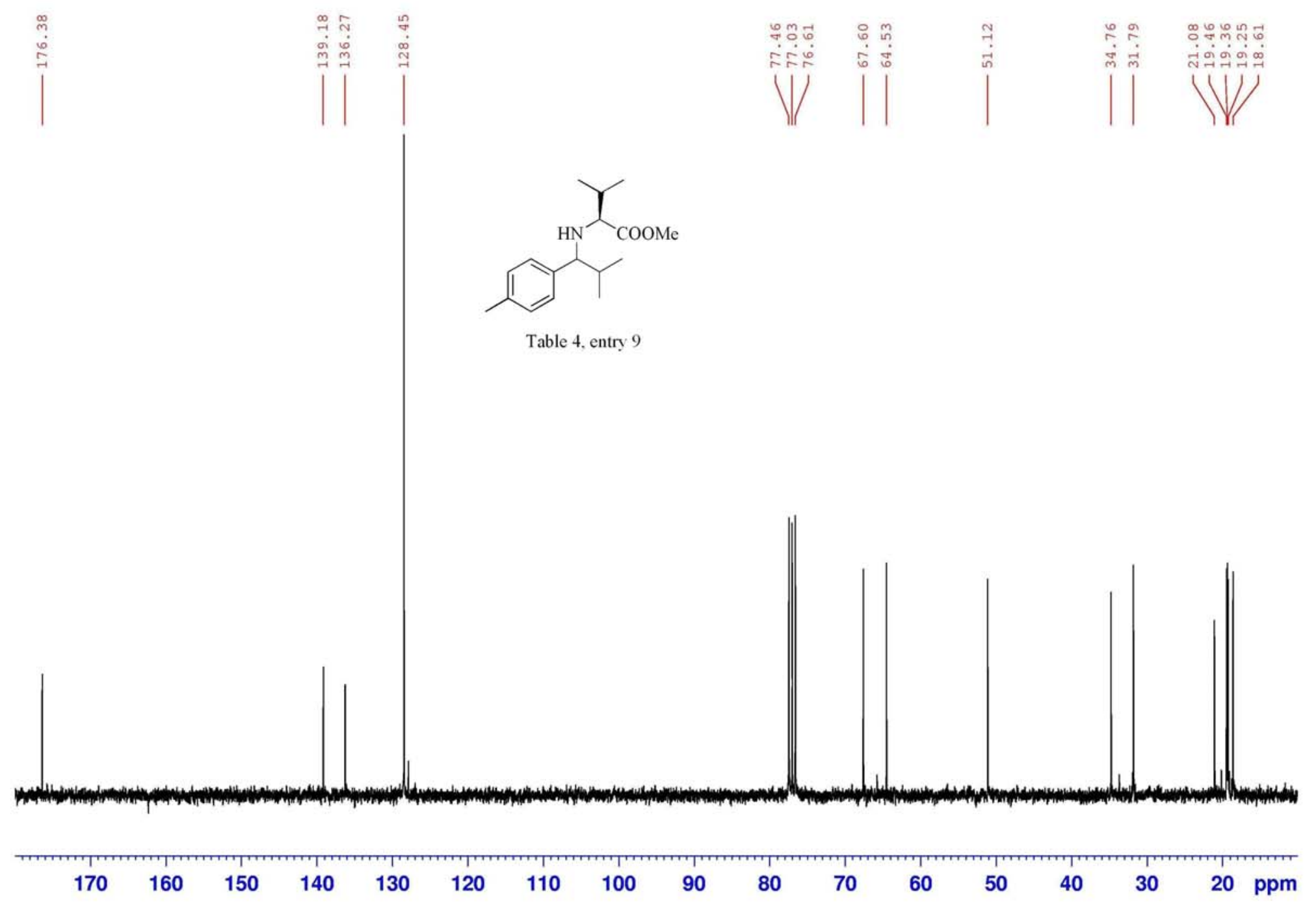




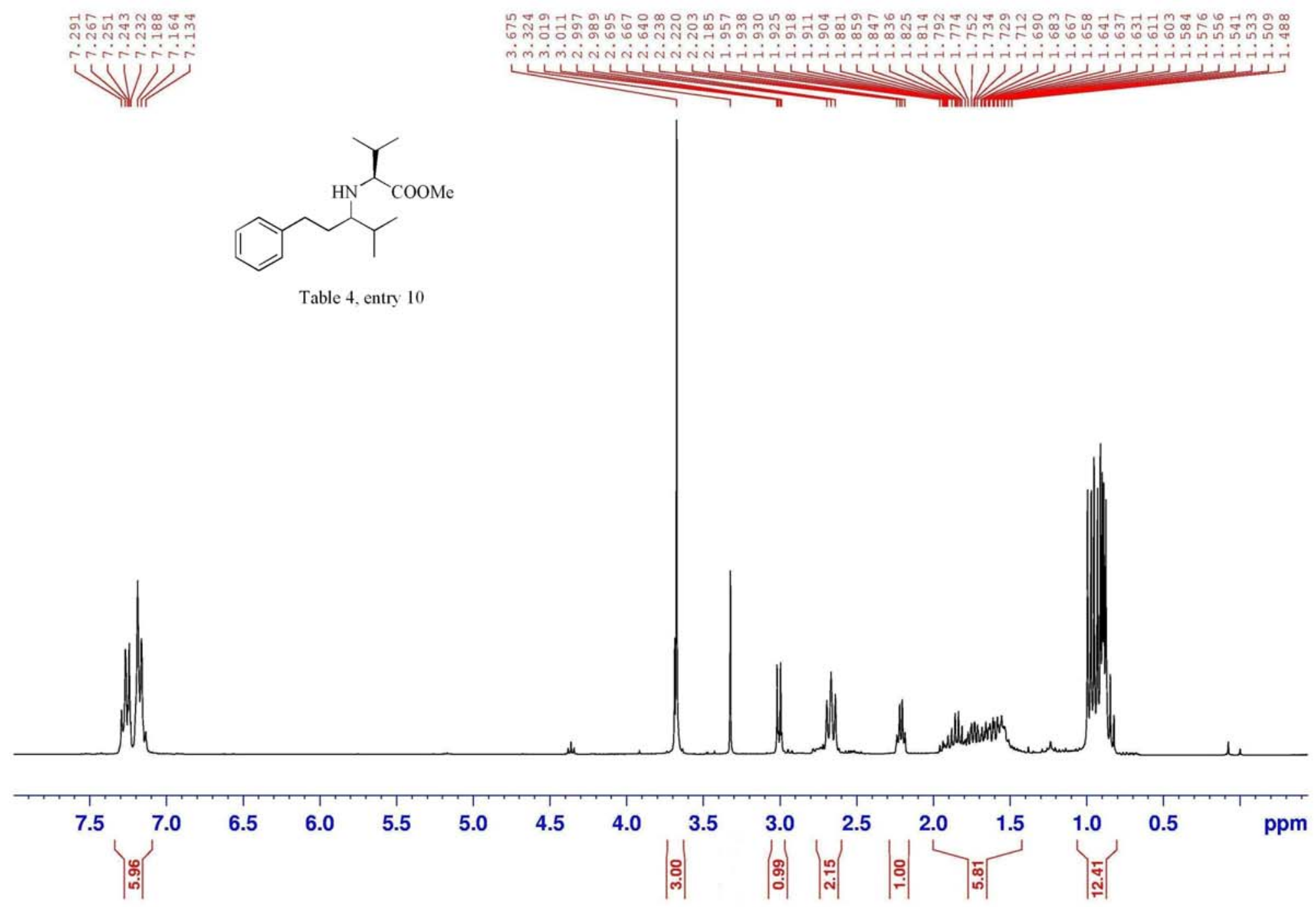




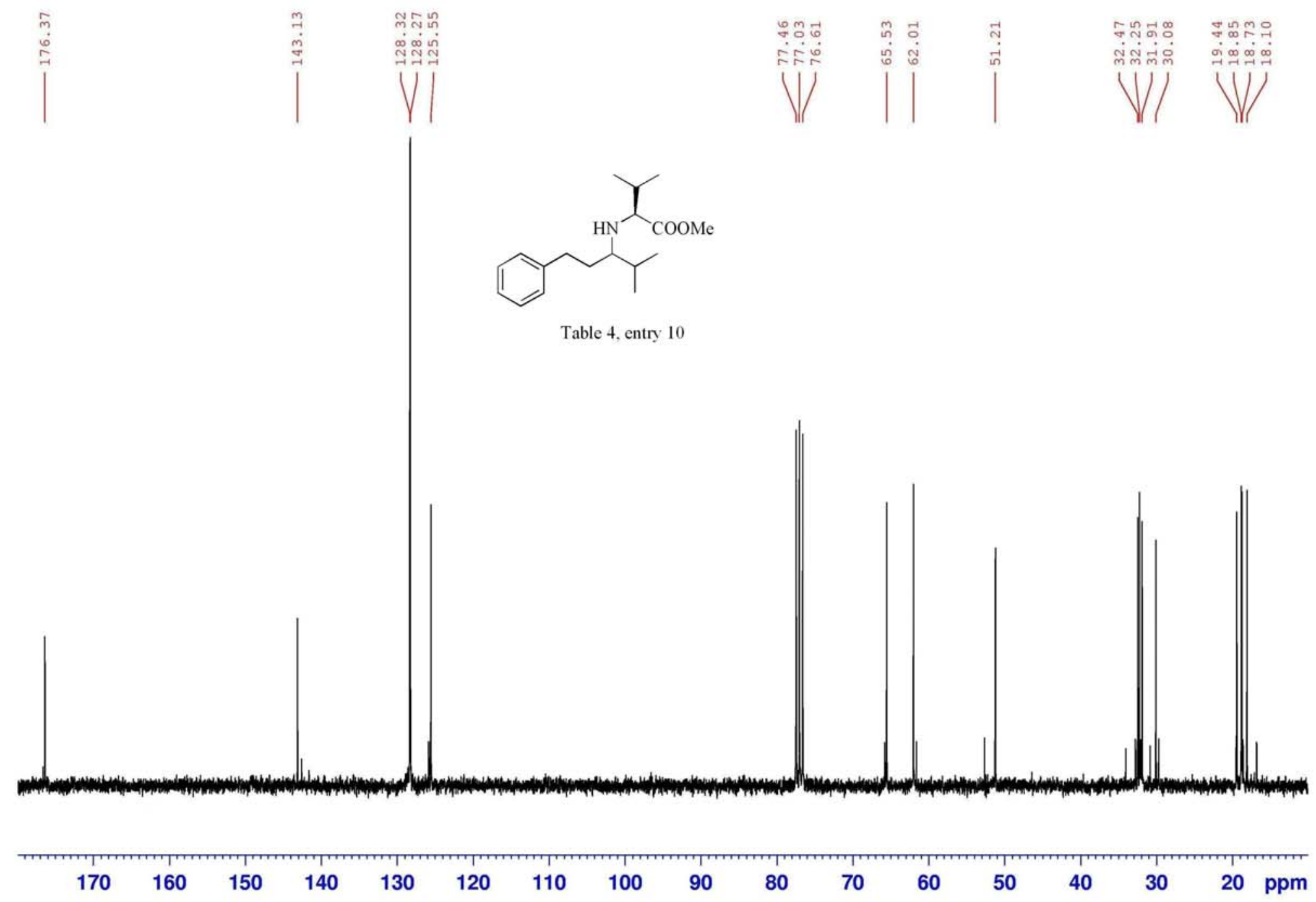




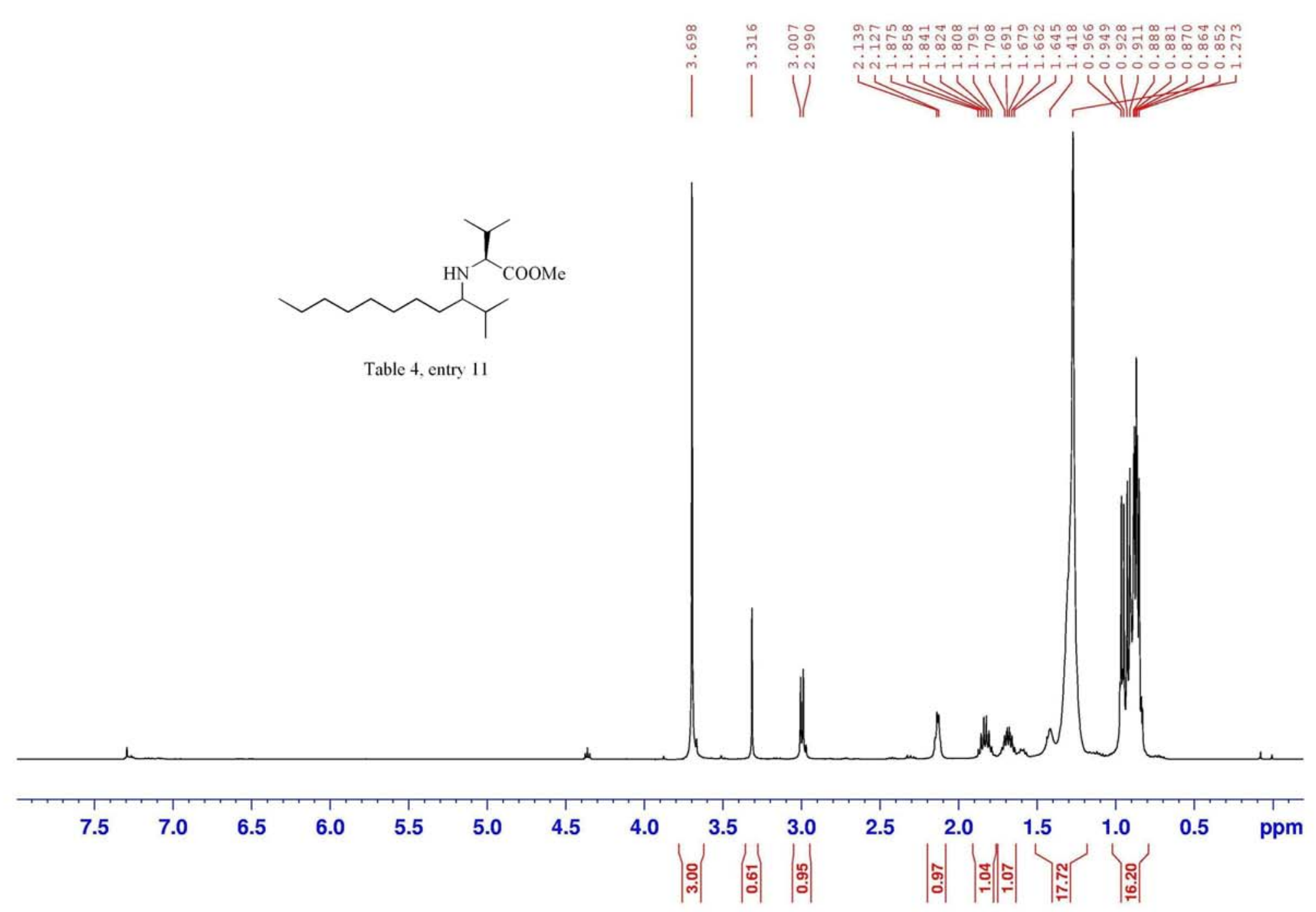




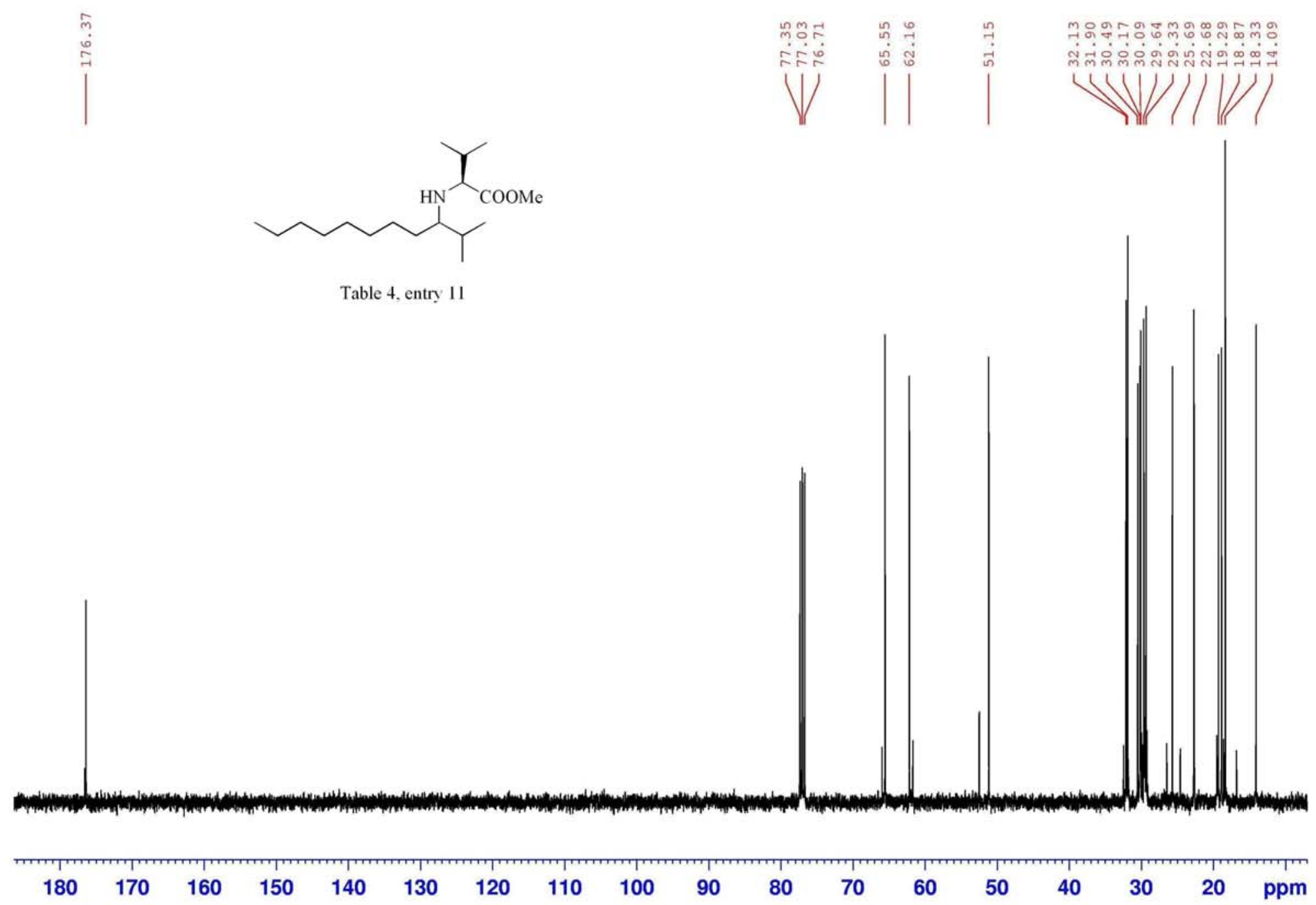




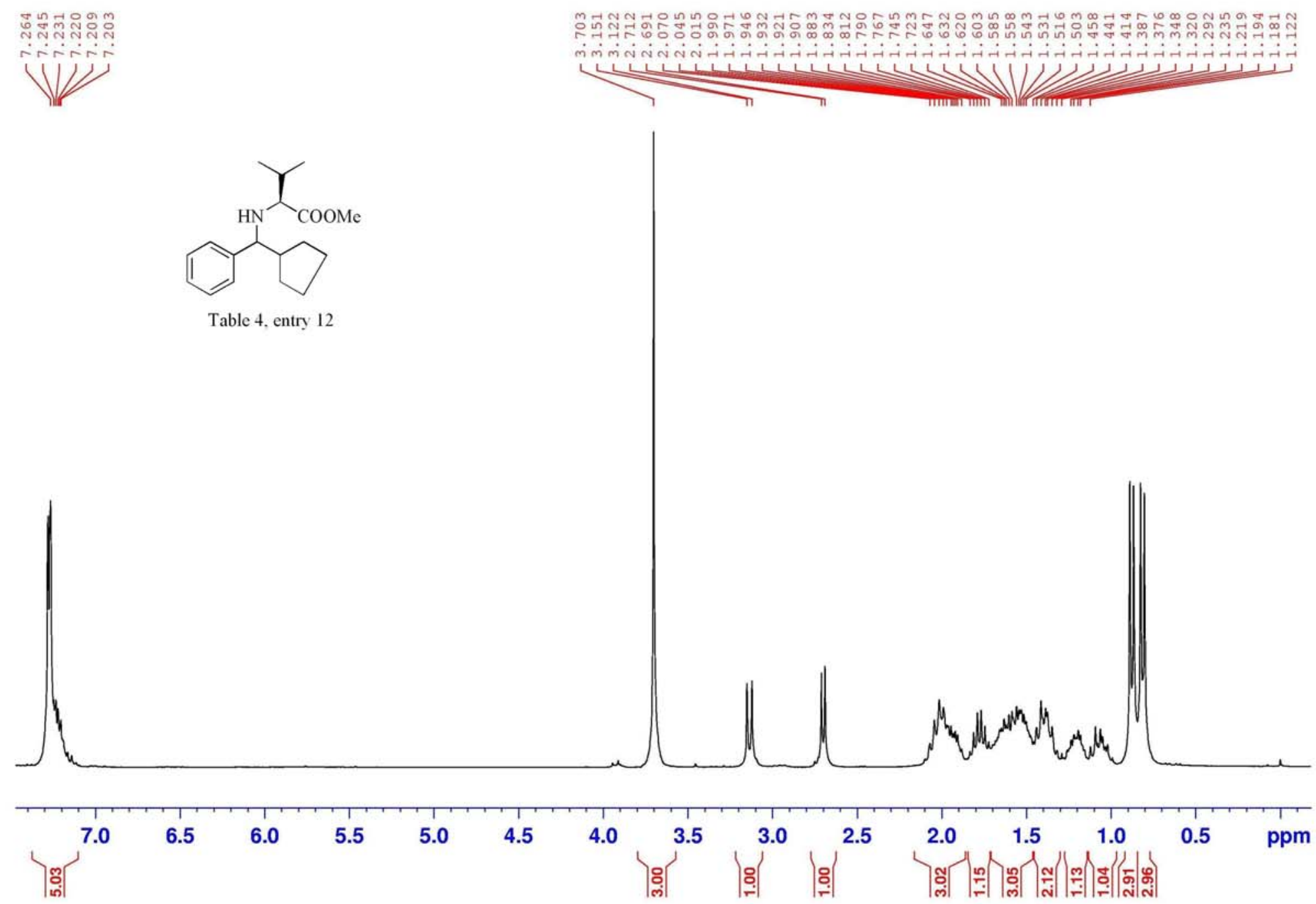




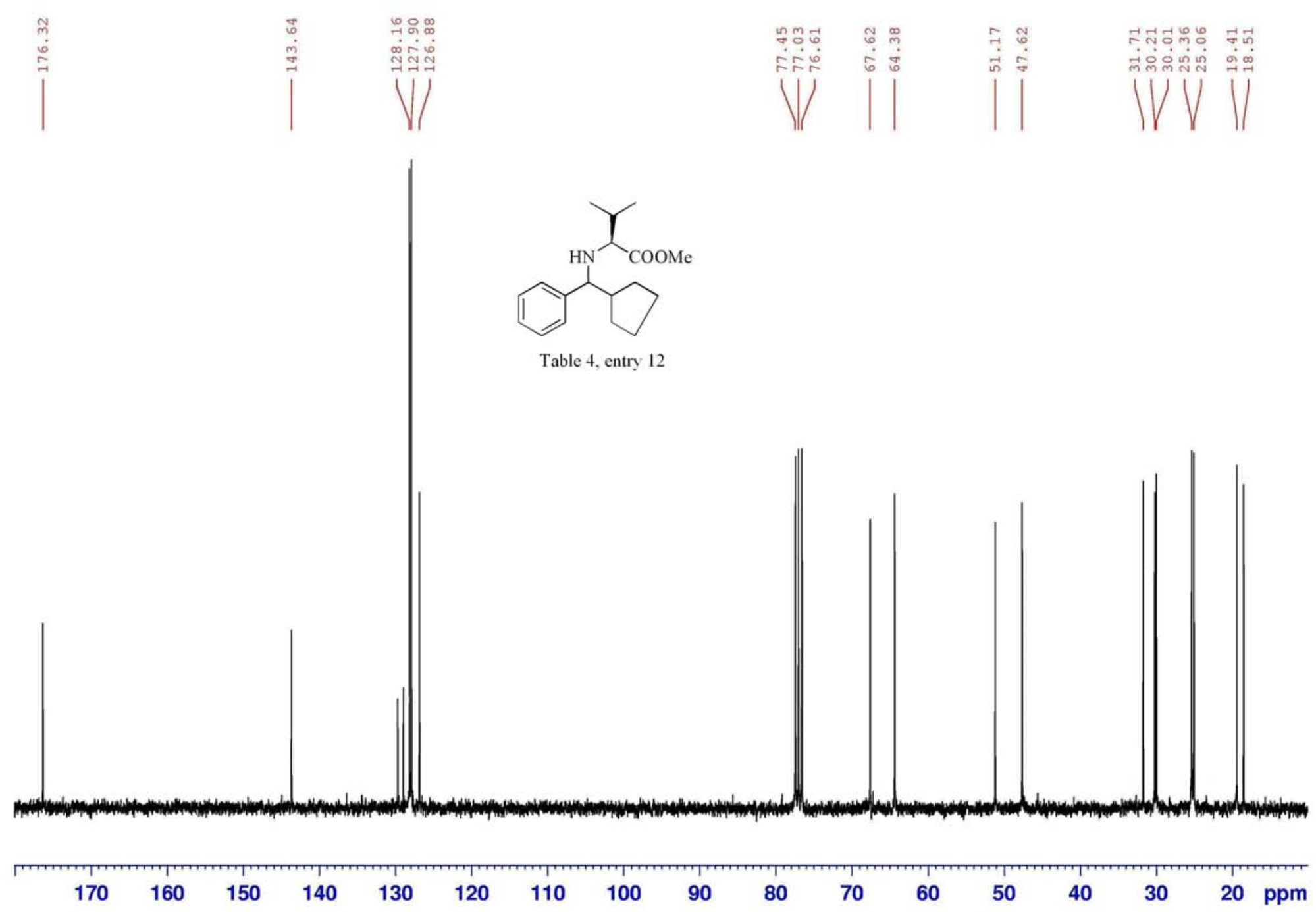




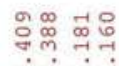 \\ î̀}

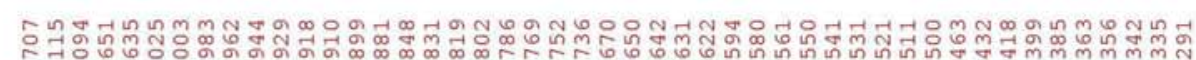
भिलंखं

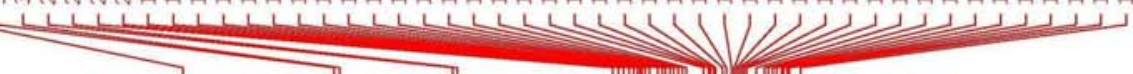

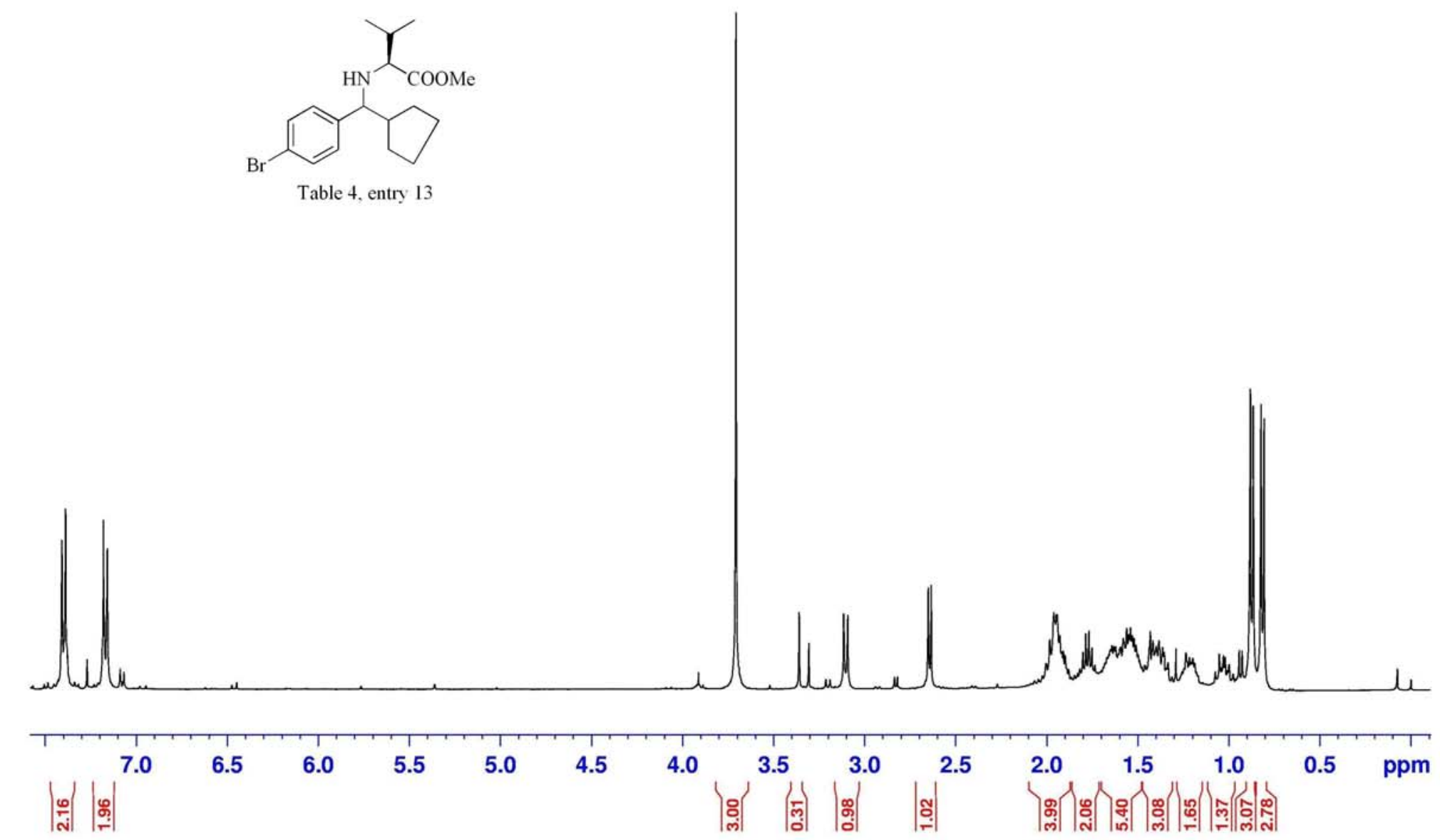




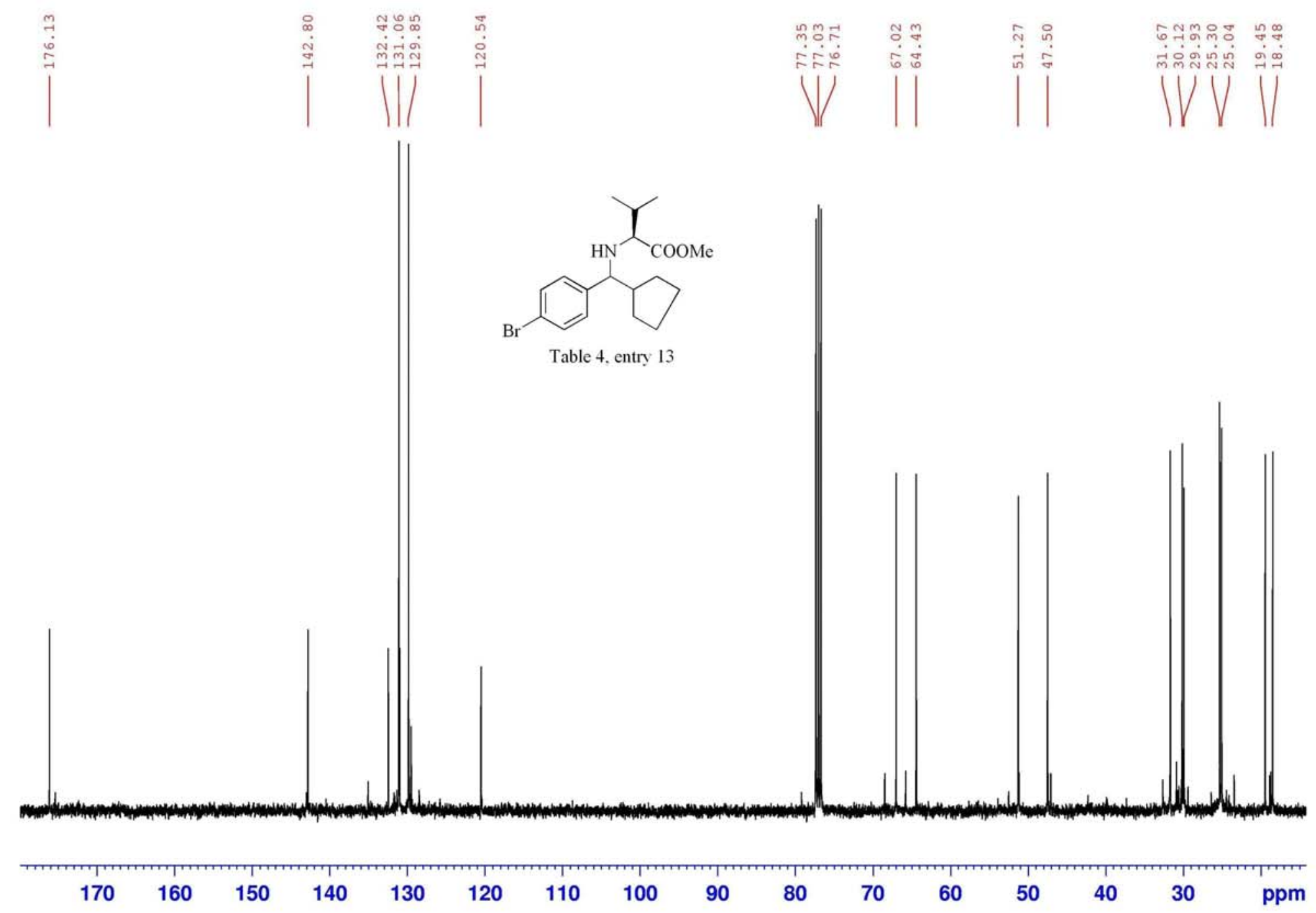




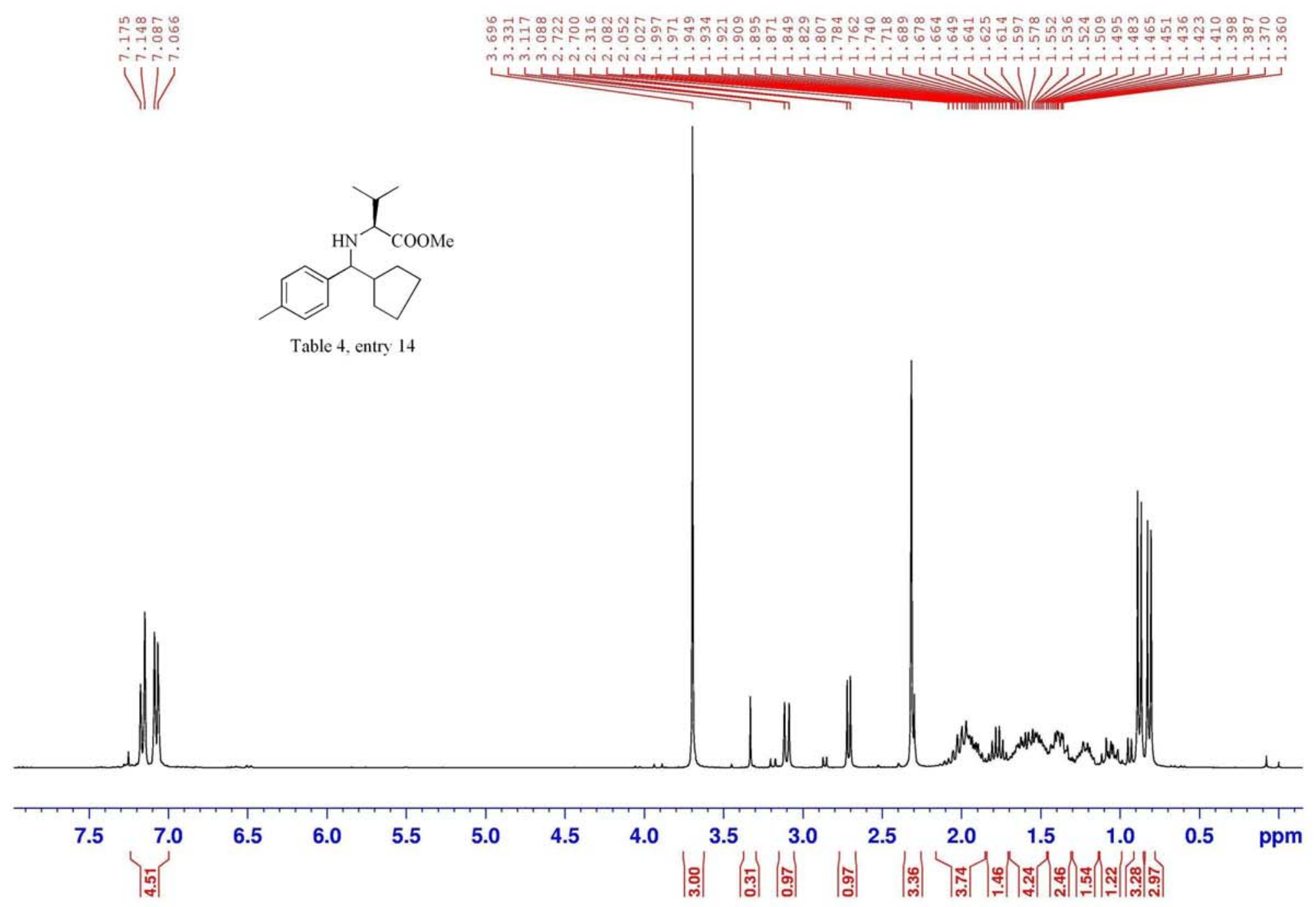




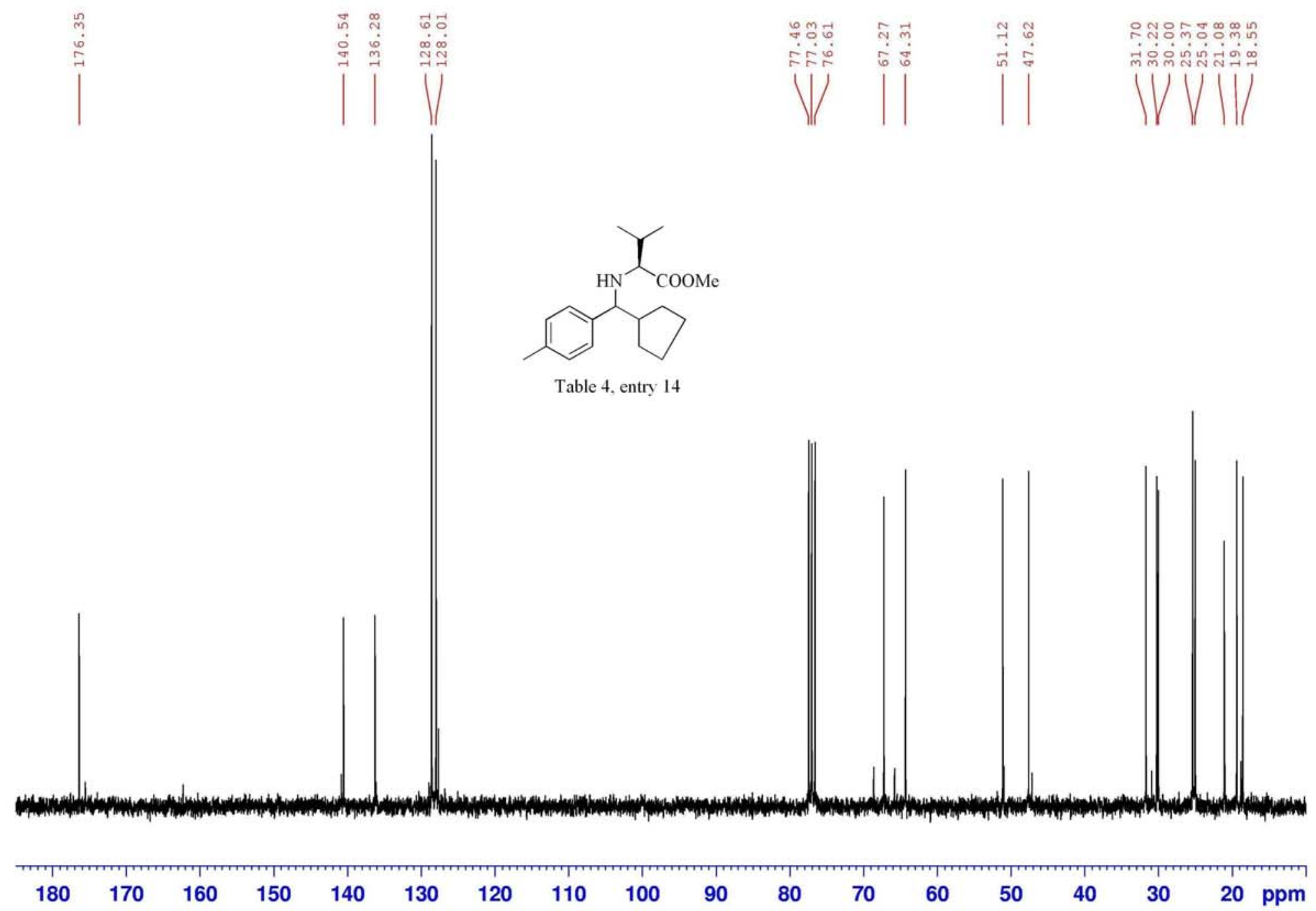




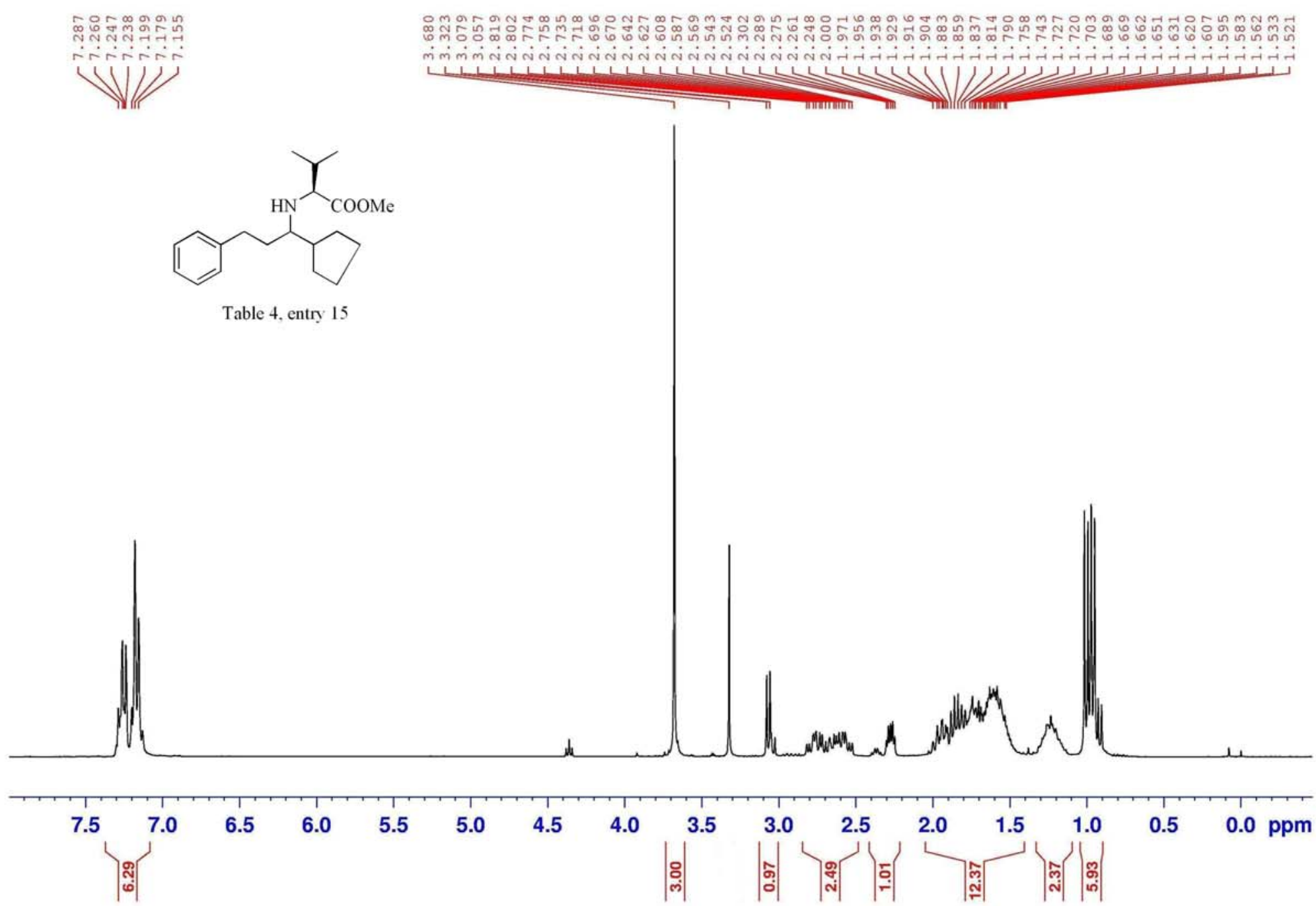



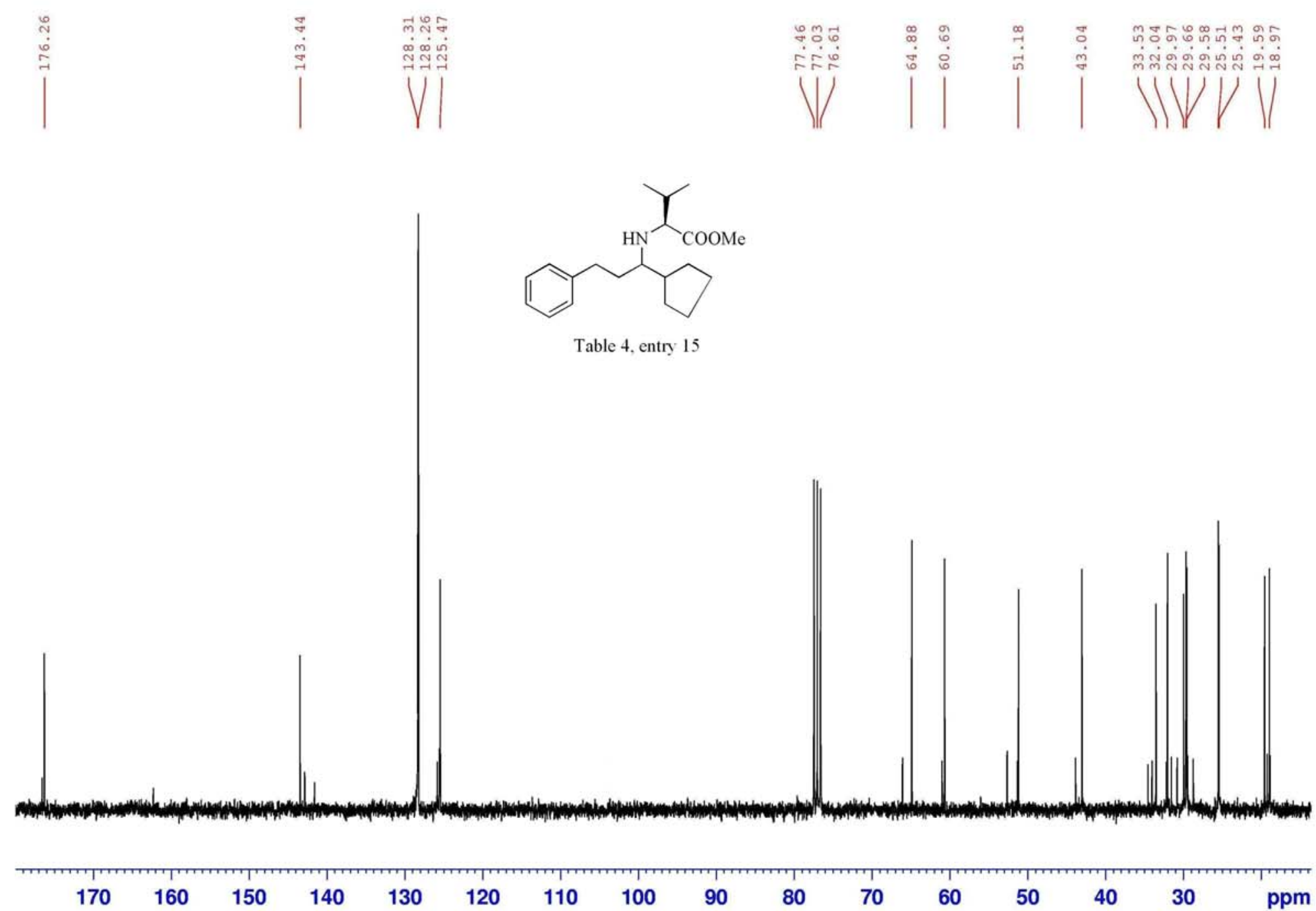

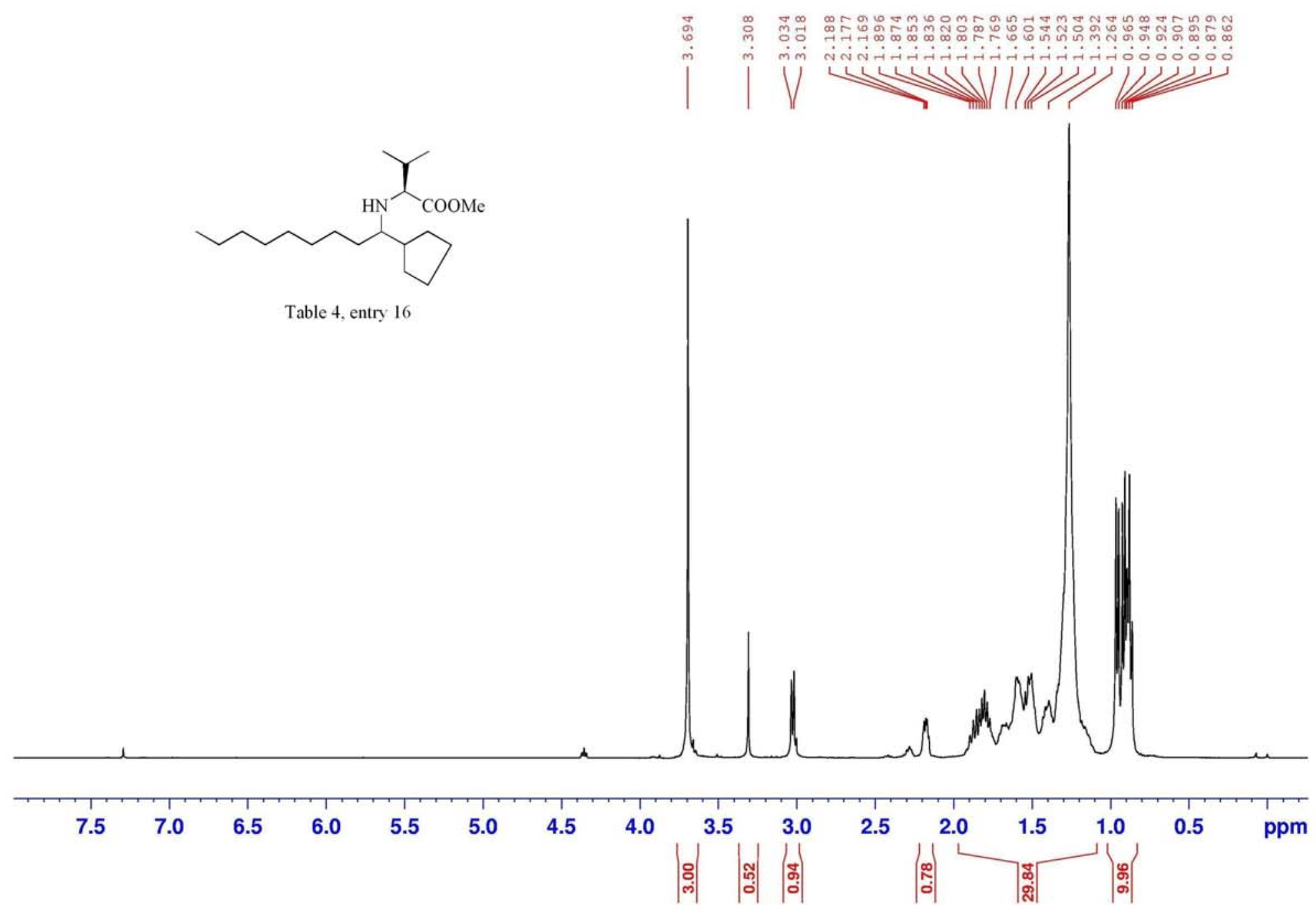


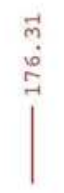

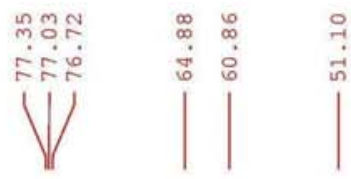

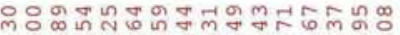

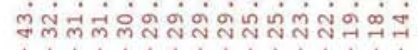

IWWVIIIII

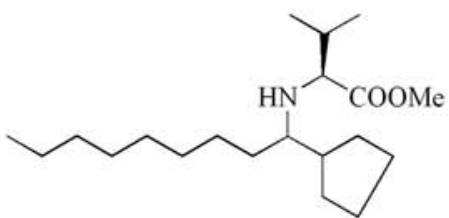

Table 4, entry 16
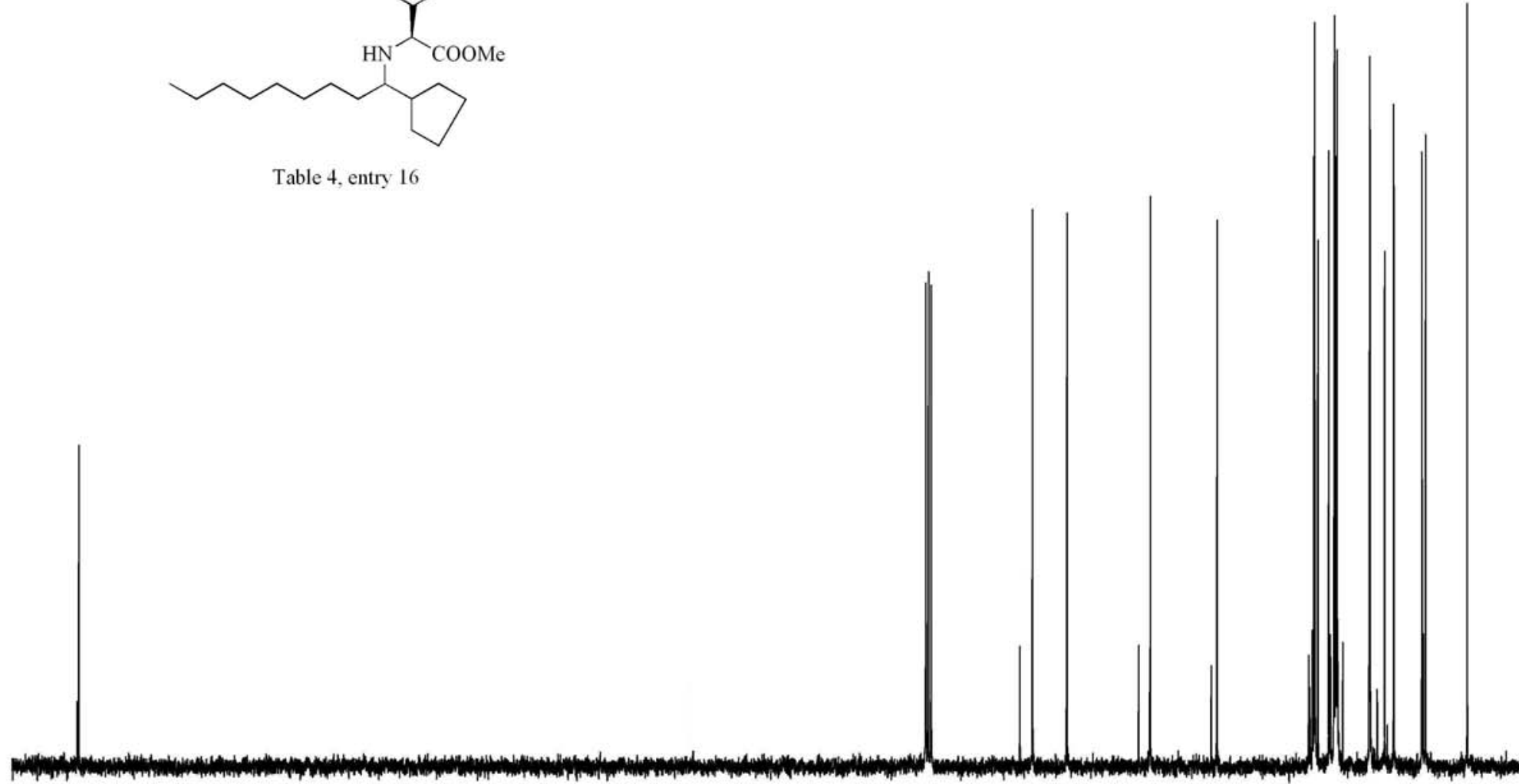